\title{
DUALIZING COMPLEXES AND PERVERSE SHEAVES ON NONCOMMUTATIVE RINGED SCHEMES
}

\author{
AMNON YEKUTIELI AND JAMES J. ZHANG
}

\begin{abstract}
A quasi-coherent ringed scheme is a pair $(X, \mathcal{A})$, where $X$ is a scheme, and $\mathcal{A}$ is a noncommutative quasi-coherent $\mathcal{O}_{X}$-ring. We introduce dualizing complexes over quasi-coherent ringed schemes and study their properties. For a separated differential quasi-coherent ringed scheme of finite type over a field, we prove existence and uniqueness of a rigid dualizing complex. In the proof we use the theory of perverse coherent sheaves in order to glue local pieces of the rigid dualizing complex into a global complex.
\end{abstract}

\section{INTRODUCTION}

The "classical" Grothendieck duality theory, dealing with dualizing complexes over schemes, was developed in the book Residues and Duality by Hartshorne [RD]. Dualizing sheaves and complexes have important roles in several areas of algebraic geometry, including moduli spaces, resolution of singularities, arithmetic geometry and enumerative geometry. Various refinements, generalizations and explicit reformulations of Grothendieck's theory have appeared since [RD; a partial list of papers is $[\mathrm{Kl}, \mathrm{Li}, \mathrm{HK}, \mathrm{Ye} 2, \mathrm{Ne}, \mathrm{AJL}$ and $\mathrm{Co}$.

A noncommutative affine duality theory was introduced in Ye1]. By "affine" we mean that this theory deals with noncommutative algebras over a base field $\mathbb{k}$. In the decade since its introduction the theory of noncommutative dualizing complexes has progressed in several directions, and it has applications in noncommutative algebraic geometry, ring theory, representation theory and even mathematical physics. Here is a sample of papers: [VdB1, [Jo, [MY], WZ, Ch2, [EG], KKO, [NV], [LR and AKO. The definition of dualizing complex over a noncommutative algebra is recalled in Section 2

The aim of this paper is to study Grothendieck duality on noncommutative spaces. As motivation one should consider the role Grothendieck duality plays both in commutative algebraic geometry and in noncommutative ring theory. Furthermore, recent developments, mainly surrounding homological mirror symmetry (cf. [BO and Do ), tell us that there is a profound interplay between algebraic geometry, noncommutative algebra and derived categories. Duality for noncommutative spaces sits right in the middle of these areas of research.

Date: 6 April 2006.

Key words and phrases. Noncommutative algebraic geometry, perverse sheaves, dualizing complexes.

Mathematics Subject Classification 2000. Primary: 14A22; Secondary: 14F05, 14J32, 16E30, 16D90, 18E30.

This research was supported by the US-Israel Binational Science Foundation. The second author was partially supported by the US National Science Foundation. 
Actually there is one type of non-affine noncommutative space for which duality has already been studied. These are the noncommutative projective schemes Proj $A$ of Artin-Zhang AZ. Here $A$ is a noetherian connected graded $\mathbb{k}$-algebra satisfying the $\chi$-condition. A global duality theory for $\operatorname{Proj} A$ was discussed in YYZ1 and KKO. However in the present paper we choose to stay closer to the RD paradigm, namely to develop a theory that has both local and global aspects.

Here are a few issues one should consider before proposing a theory of Grothendieck duality on noncommutative spaces. The first is to decide what is meant by a noncommutative space - there are several reasonable choices in current literature. The second is to find a suitable formulation of duality, which shall include in a natural way the established commutative and noncommutative theories. The third issue is whether this duality theory applies to a wide enough class of spaces.

The noncommutative spaces we shall concentrate on are the noetherian quasicoherent ringed schemes. A quasi-coherent ringed scheme is a pair $(X, \mathcal{A})$, where $X$ is a scheme over $\mathbb{k}$, and $\mathcal{A}$ is a (possibly noncommutative) quasi-coherent $\mathcal{O}_{X}$-ring (see Definition 1.1). This type of geometric object includes schemes $\left(\mathcal{A}=\mathcal{O}_{X}\right)$, noncommutative rings $(X=\operatorname{Spec} \mathbb{k})$ and rings of differential operators $\left(\mathcal{A}=\mathcal{D}_{X}\right)$. Our definition of dualizing complex extends the established definitions in all these cases. A dualizing complex $\mathcal{R}$ over $(X, \mathcal{A})$ is an object of the derived category $\mathrm{D}\left(\operatorname{Mod} \mathcal{A}^{\mathrm{e}}\right)$, where $\left(X^{2}, \mathcal{A}^{\mathrm{e}}\right)$ is the product of $(X, \mathcal{A})$ and its opposite $\left(X, \mathcal{A}^{\mathrm{op}}\right)$. The duality functor $\mathrm{D}: \mathrm{D}_{\mathrm{c}}^{\mathrm{b}}(\operatorname{Mod} \mathcal{A}) \rightarrow \mathrm{D}_{\mathrm{c}}^{\mathrm{b}}\left(\operatorname{Mod} \mathcal{A}^{\mathrm{op}}\right)$ is a contravariant Fourier-Mukai transform with respect to $\mathcal{R}$. See Definitions 1.3 and 2.3 for details.

We shall restrict our attention to dualizing complexes that have a "local" behavior, namely those that are supported on the diagonal $\Delta(X) \subset X^{2}$. Moreover we are interested in dualizing complexes that are canonical, or functorial, in a suitable sense - something resembling Grothendieck's dualizing complex $\pi^{\prime} \mathbb{k} \in$ $\mathrm{D}_{\mathrm{c}}^{\mathrm{b}}\left(\operatorname{Mod} \mathcal{O}_{X}\right)$, where $\pi: X \rightarrow$ Spec $\mathbb{k}$ is the structural morphism (see [RD]). Thus we propose to consider rigid dualizing complexes.

Let $A$ be a noetherian $\mathbb{k}$-algebra. A rigid dualizing complex over $A$ is a dualizing complex $R_{A}$ equipped with an isomorphism

$$
\rho_{A}: R_{A} \stackrel{\simeq}{\rightarrow} \operatorname{RHom}_{A^{\mathrm{e}}}\left(A, R_{A} \otimes R_{A}\right)
$$

in $\mathrm{D}\left(\operatorname{Mod} A^{\mathrm{e}}\right)$, called a rigidifying isomorphism. This notion is due to Van den Bergh VdB1. It is known that the pair $\left(R_{A}, \rho_{A}\right)$ is unique up to a unique isomorphism in $\mathrm{D}\left(\operatorname{Mod} A^{\mathrm{e}}\right)$.

Passing to sheaves, a rigid dualizing complex over $\mathcal{A}$ is a dualizing complex $\mathcal{R}_{\mathcal{A}}$ supported on the diagonal in $X^{2}$, together with a collection $\boldsymbol{\rho}=\left\{\rho_{U}\right\}$ of rigidifying isomorphisms, indexed by the affine open sets $U \subset X$. For any such $U$ the complex $R_{A}:=\mathrm{R} \Gamma\left(U^{2}, \mathcal{R}_{\mathcal{A}}\right)$ is a dualizing complex over the ring $A:=\Gamma(U, \mathcal{A})$, and $\rho_{A}:=\rho_{U}$ is a rigidifying isomorphism for $R_{A}$. Moreover, the isomorphisms $\rho_{U}$ are required to satisfy a compatibility condition (see Definition 3.7).

Our main objective in this paper is to prove existence and uniqueness of a rigid dualizing complex over $\mathcal{A}$ (under suitable assumptions).

A class of noncommutative spaces for which this can be done is that of separated differential quasi-coherent ringed schemes of finite type over $\mathbb{k}$. Suppose $(X, \mathcal{A})$ is such a space. By definition $X$ is a separated finite type $\mathbb{k}$-scheme, and $\mathcal{A}$ has a filtration such that the graded ring gr $\mathcal{A}$ is a finite type quasi-coherent $\mathcal{O}_{X}$-algebra with a big center. Such a filtration is called a differential filtration. See Definition 5.2 for details. The prototypical examples are: 
(1) $\mathcal{A}$ is a coherent $\mathcal{O}_{X}$-algebra (e.g. $\mathcal{A}=\mathcal{O}_{X}$, or $\mathcal{A}$ is an Azumaya algebra with center $\mathcal{O}_{X}$ );

(2) $\mathcal{A}$ is the ring $\mathcal{D}_{X}$ of differential operators on a smooth scheme $X$ in characteristic 0 ; and

(3) $\mathcal{A}$ is the universal enveloping algebra $\mathrm{U}\left(\mathcal{O}_{X}, \mathcal{L}\right)$ of a coherent Lie algebroid $\mathcal{L}$ on $X$.

In (1) and (3) there are no regularity assumptions on $X, \mathcal{A}$ or $\mathcal{L}$. More examples are provided in Section 6

Suppose $(X, \mathcal{A})$ is a differential quasi-coherent ringed scheme of finite type, $U$ is an affine open set and $A:=\Gamma(U, \mathcal{A})$. The ring $A$ is then a differential $\mathbb{k}$-algebra of finite type. In YZ4 we proved that $A$ has a rigid dualizing complex $R_{A}$, which is supported on the diagonal in $U^{2}$.

The fact that $R_{A}$ is supported on the diagonal implies that it sheafifies to a complex $\mathcal{R}_{\left.\mathcal{A}\right|_{U}} \in \mathrm{D}\left(\left.\operatorname{Mod} \mathcal{A}^{\mathrm{e}}\right|_{U^{2}}\right)$, which is a dualizing complex over $\left(U,\left.\mathcal{A}\right|_{U}\right)$. Because of the uniqueness of rigid dualizing complexes we obtain isomorphisms

$$
\left.\left.\mathcal{R}_{\left.\mathcal{A}\right|_{U_{1}}}\right|_{U_{1}^{2} \cap U_{2}^{2}} \cong \mathcal{R}_{\left.\mathcal{A}\right|_{U_{2}}}\right|_{U_{1}^{2} \cap U_{2}^{2}}
$$

in $\mathrm{D}\left(\left.\operatorname{Mod} \mathcal{A}^{\mathrm{e}}\right|_{U_{1}^{2} \cap U_{2}^{2}}\right)$ for pairs of affine open sets $U_{1}$ and $U_{2}$, and these isomorphisms satisfy the cocycle condition on triple intersections.

The next stage is a process of gluing. Here we encounter a genuine problem: usually objects in derived categories cannot be glued. Grothendieck's solution in the commutative case, in [RD, was to use Cousin complexes. However, as explained in YZ3, this solution seldom applies in the noncommutative context. The discovery at the heart of our present paper is that perverse coherent sheaves can be used instead of Cousin complexes for gluing dualizing complexes.

The concepts of t-structure and perverse sheaf are due to Bernstein, Beilinson and Deligne $\mathrm{BBD}$, and we recall the definitions in Section 4 It turns out that rigid dualizing complexes give rise to a perverse t-structure on $\mathrm{D}_{\mathrm{c}}^{\mathrm{b}}(\operatorname{Mod} \mathcal{A})$. Indeed, for any affine open set $U$ we have a rigid dualizing complex $R_{A}$ over $A:=\Gamma(U, \mathcal{A})$. Let $\mathrm{D}: \mathrm{D}_{\mathrm{f}}^{\mathrm{b}}(\operatorname{Mod} A) \rightarrow \mathrm{D}_{\mathrm{f}}^{\mathrm{b}}\left(\operatorname{Mod} A^{\mathrm{op}}\right)$ be the duality functor $\mathrm{RHom}_{A}\left(-, R_{A}\right)$. The rigid perverse $t$-structure on $\mathrm{D}_{\mathrm{c}}^{\mathrm{b}}(\operatorname{Mod} A)$ is defined by

$$
\begin{aligned}
& { }^{p} \mathrm{D}_{\mathrm{c}}^{\mathrm{b}}(\operatorname{Mod} A)^{\leq 0}:=\left\{M \mid \mathrm{H}^{i} \mathrm{D} M=0 \text { for all } i<0\right\}, \\
& { }^{p} \mathrm{D}_{\mathrm{c}}^{\mathrm{b}}(\operatorname{Mod} A)^{\geq 0}:=\left\{M \mid \mathrm{H}^{i} \mathrm{D} M=0 \text { for all } i>0\right\} .
\end{aligned}
$$

The intersection of these subcategories (the heart) is denoted by ${ }^{p} \mathrm{D}_{\mathrm{c}}^{\mathrm{b}}(\operatorname{Mod} A)^{0}$. Next let " $\star$ " denote either " $\leq 0$ ", " $\geq 0$ " or " 0 ", and define

$$
{ }^{p} \mathrm{D}_{\mathrm{c}}^{\mathrm{b}}(\operatorname{Mod} \mathcal{A})^{\star}:=\left\{\mathcal{M} \in \mathrm{D}_{\mathrm{c}}^{\mathrm{b}}(\operatorname{Mod} \mathcal{A}) \mid \mathrm{R} \Gamma(U, \mathcal{M}) \in{ }^{p} \mathrm{D}_{\mathrm{f}}^{\mathrm{b}}(\operatorname{Mod} \Gamma(U, \mathcal{A}))^{\star}\right.
$$

for all affine open sets $U\}$.

Here is the first main result of the paper.

Theorem 0.2. Let $(X, \mathcal{A})$ be a differential quasi-coherent ringed $\mathbb{k}$-scheme of finite type. Then:

(1) The pair

$$
\left({ }^{p} \mathrm{D}_{\mathrm{c}}^{\mathrm{b}}(\operatorname{Mod} \mathcal{A}) \leq 0,{ }^{p} \mathrm{D}_{\mathrm{c}}^{\mathrm{b}}(\operatorname{Mod} \mathcal{A})^{\geq 0}\right)
$$

is a t-structure on $\mathrm{D}_{\mathrm{c}}^{\mathrm{b}}(\operatorname{Mod} \mathcal{A})$.

(2) The assignment $V \mapsto{ }^{p} \mathrm{D}_{\mathrm{c}}^{\mathrm{b}}\left(\left.\operatorname{Mod} \mathcal{A}\right|_{V}\right)^{0}$, for $V \subset X$ open, is a stack of abelian categories on $X$. 
Part (2) of the theorem says that ${ }^{p} \mathrm{D}_{\mathrm{c}}^{\mathrm{b}}(\operatorname{Mod} \mathcal{A})^{0}$ behaves like the category of coherent sheaves $\operatorname{Coh} \mathcal{A}$; hence its objects are called perverse coherent sheaves. The theorem is restated as Theorem 5.9

It is not hard to show that the product $\left(X^{2}, \mathcal{A}^{\mathrm{e}}\right)$ exists, and that it too is a differential quasi-coherent ringed scheme of finite type. So by Theorem 0.2 the rigid perverse t-structure on $\mathrm{D}_{\mathrm{c}}^{\mathrm{b}}\left(\operatorname{Mod} \mathcal{A}^{\mathrm{e}}\right)$ exists, and perverse $\mathcal{A}^{\mathrm{e}}$-modules can be glued.

According to YZ4, for every affine open set $U$ the complex $\mathcal{R}_{\left.\mathcal{A}\right|_{U}}$ is a perverse

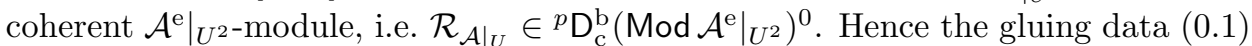
gives rise to a global dualizing complex $\mathcal{R}_{\mathcal{A}}$. Thus we obtain the second main result of our paper (which is repeated as Theorem [5.11):

Theorem 0.3. Let $(X, \mathcal{A})$ be a separated differential quasi-coherent ringed $\mathbb{k}$ scheme of finite type. Then there exists a rigid dualizing complex $\left(\mathcal{R}_{\mathcal{A}}, \boldsymbol{\rho}\right)$ over $\mathcal{A}$. It is unique up to a unique isomorphism in $\mathrm{D}_{\mathrm{c}}^{\mathrm{b}}\left(\operatorname{Mod} \mathcal{A}^{\mathrm{e}}\right)$.

We also prove that the rigid trace exists for a finite centralizing morphism $f$ : $(Y, \mathcal{B}) \rightarrow(X, \mathcal{A}) ;$ see Theorem 5.15

Here is a section-by-section synopsis of the paper. In Section 1 we define quasicoherent ringed schemes, discuss criteria for existence of the product $(X, \mathcal{A}) \times(Y, \mathcal{B})$ of two quasi-coherent ringed schemes, and consider some properties of the derived category $\mathrm{D}(\operatorname{Mod} \mathcal{A})$. In Section 2 we define dualizing complexes over quasi-coherent ringed schemes, and present a couple of "exotic" examples. Section 3 is about rigid dualizing complexes: their definition and some properties. In Section 4 we study t-structures on ringed spaces. The main result here is that a local collection of tstructures can be glued to a global t-structure (Theorems 4.14 and 4.15). In Section 5 we put together all the previous results to prove Theorem 0.3 Finally, in Section 6 we give examples of differential quasi-coherent ringed schemes and their rigid dualizing complexes. We also take a close look at the commutative case $\mathcal{A}=\mathcal{O}_{X}$.

Acknowledgments. The authors wish to thank Eitan Bachmat, Joseph Bernstein, Sophie Chemla, Masaki Kashiwara, David Kazhdan, Maxim Kontsevich, Thierry Levasseur and Michel Van den Bergh for helpful conversations. We also thank the referee for reading the paper carefully and suggesting some corrections.

\section{Quasi-Coherent Ringed Schemes}

Throughout the paper $\mathbb{k}$ is a base field. All rings are by default $\mathbb{k}$-algebras, and all bimodules are central over $\mathbb{k}$. Let $(X, \mathcal{A})$ be a ringed space over $\mathbb{k}$. Thus $X$ is a topological space, and $\mathcal{A}$ is a sheaf of (possibly noncommutative) $\mathbb{k}$-algebras on $X$. By an $\mathcal{A}$-bimodule we mean a sheaf $\mathcal{M}$ of $\mathbb{k}$-modules on $X$, together with a left $\mathcal{A}$-module structure and a right $\mathcal{A}$-module structure that commute with each other. In other words $\mathcal{M}$ is a module over the sheaf of rings $\mathcal{A} \otimes_{\mathbb{k}_{X}} \mathcal{A}^{\text {op }}$, where $\mathbb{k}_{X}$ is the constant sheaf $\mathbb{k}$ on $X$. An $\mathcal{A}$-ring is a sheaf $\mathcal{B}$ of rings on $X$ together with a ring homomorphism $\mathcal{A} \rightarrow \mathcal{B}$. Note that $\mathcal{B}$ is an $\mathcal{A}$-bimodule.

Definition 1.1. (1) A ringed scheme over $\mathbb{k}$ is a pair $(X, \mathcal{A})$ consisting of a $\mathbb{k}$-scheme $X$ and an $\mathcal{O}_{X}$-ring $\mathcal{A}$.

(2) We say $\mathcal{A}$ is a quasi-coherent $\mathcal{O}_{X}$-ring, and the pair $(X, \mathcal{A})$ is a quasicoherent ringed scheme, if the $\mathcal{O}_{X^{-}}$-bimodule $\mathcal{A}$ is a quasi-coherent $\mathcal{O}_{X^{-}}$ module on both sides. 
(3) A quasi-coherent ringed scheme $(X, \mathcal{A})$ is called separated (resp. affine) if $X$ is a separated (resp. affine) $\mathbb{k}$-scheme.

Henceforth in this section we consider quasi-coherent ringed schemes.

Definition 1.2. A morphism $(f, \psi):(Y, \mathcal{B}) \rightarrow(X, \mathcal{A})$ of quasi-coherent ringed schemes over $\mathbb{k}$ is a morphism of schemes $f: Y \rightarrow X$, together with a homomorphism $\psi: \mathcal{A} \rightarrow f_{*} \mathcal{B}$ of $\mathcal{O}_{X}$-rings.

Often we will use an abbreviated form, and denote by $f^{*}$ both the homomorphism $\mathcal{O}_{X} \rightarrow f_{*} \mathcal{O}_{Y}$ and the homomorphism $\psi: \mathcal{A} \rightarrow f_{*} \mathcal{B}$.

Suppose we are given two quasi-coherent ringed schemes $\left(X_{1}, \mathcal{A}_{1}\right)$ and $\left(X_{2}, \mathcal{A}_{2}\right)$ over $\mathbb{k}$. Let us denote by $X_{1} \times X_{2}:=X_{1} \times_{\mathbb{k}} X_{2}$ the usual product of schemes, and by $\mathrm{p}_{i}: X_{1} \times X_{2} \rightarrow X_{i}$ the projections. We obtain sheaves of $\mathbb{k}_{X \times Y}$-algebras $\mathrm{p}_{i}^{-1} \mathcal{O}_{X_{i}}$ and $\mathrm{p}_{i}^{-1} \mathcal{A}_{i}$, and there are canonical ring homomorphisms:

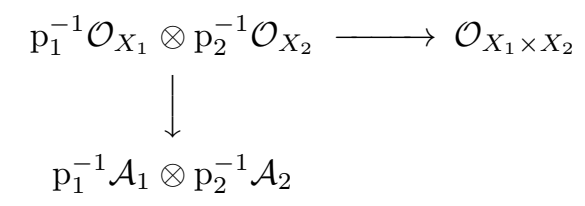

where " $\otimes$ " denotes " $\otimes_{\mathbb{k}_{X \times Y}}$ ".

Definition 1.3. Let $\left(X_{1}, \mathcal{A}_{1}\right)$ and $\left(X_{2}, \mathcal{A}_{2}\right)$ be two quasi-coherent ringed schemes over $\mathbb{k}$. Their product is a quasi-coherent ringed scheme $\left(X_{1} \times X_{2}, \mathcal{A}_{1} \otimes \mathcal{A}_{2}\right)$, together with a ring homomorphism

$$
\phi: \mathrm{p}_{1}^{-1} \mathcal{A}_{1} \otimes \mathrm{p}_{2}^{-1} \mathcal{A}_{2} \rightarrow \mathcal{A}_{1} \otimes \mathcal{A}_{2},
$$

satisfying the conditions below.

(i) The diagram

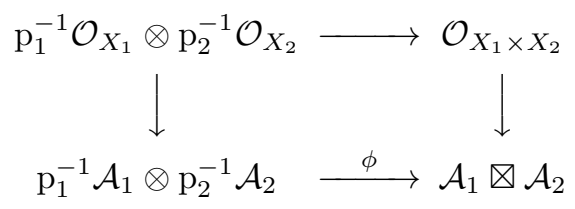

commutes.

(ii) For every pair of affine open sets $U_{1} \subset X_{1}$ and $U_{2} \subset X_{2}$ the homomorphism

$$
\Gamma\left(U_{1}, \mathcal{A}_{1}\right) \otimes \Gamma\left(U_{2}, \mathcal{A}_{2}\right) \rightarrow \Gamma\left(U_{1} \times U_{2}, \mathcal{A}_{1} \otimes \mathcal{A}_{2}\right)
$$

induced by $\phi$ is bijective.

We remind that a denominator set $S$ in a ring $A$ is a multiplicatively closed subset satisfying the left and right Ore conditions and the left and right torsion conditions. Equivalently, it means that the (left and right) ring of fractions $A_{S}=S^{-1} A=A S^{-1}$ exists. See [MR, Section 2.1] for more details.

Lemma 1.4. Let $(U, \mathcal{A})$ and $(V, \mathcal{B})$ be two affine quasi-coherent ringed schemes, $f: V \rightarrow U$ a morphism of schemes and $\psi: \Gamma(U, \mathcal{A}) \rightarrow \Gamma(V, \mathcal{B})$ a homomorphism of $\Gamma\left(U, \mathcal{O}_{U}\right)$-rings. Then there is a unique homomorphism of $\mathcal{O}_{U}$-rings $\tilde{\psi}: \mathcal{A} \rightarrow f_{*} \mathcal{B}$ such that $\Gamma(U, \tilde{\psi})=\psi$. 
Proof. Write $C:=\Gamma\left(U, \mathcal{O}_{U}\right), A:=\Gamma(U, \mathcal{A})$ and $B:=\Gamma(V, \mathcal{B})$. Choose an element $s \in C$, and define the multiplicatively closed sets $S:=\left\{s^{i}\right\}_{i \in \mathbb{Z}} \subset C, \bar{S}$ the image of $S$ in $A$ and $\bar{T}:=\psi(\bar{S}) \subset B$. Also let $U^{\prime}:=\{s \neq 0\} \subset U$ and $V^{\prime}:=\{\psi(s) \neq$ $0\} \subset V$. According to [YZ4 Corollary 5.13], $\bar{S}$ and $\bar{T}$ are denominator sets in $A$ and $B$ respectively. By the universal property of localization there is a unique

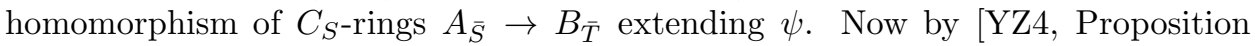
5.6] we have $A_{\bar{S}} \cong \Gamma\left(U^{\prime}, \mathcal{A}\right)$ and $B_{\bar{T}} \cong \Gamma\left(V^{\prime}, \mathcal{B}\right) \cong \Gamma\left(U^{\prime}, f_{*} \mathcal{B}\right)$. Since the open sets $U^{\prime}$, as we change $s$, form a basis of the topology of $U$, we obtain an $\mathcal{O}_{U}$-ring homomorphism $\tilde{\psi}: \mathcal{A} \rightarrow f_{*} \mathcal{B}$.

The lemma says that $(f, \tilde{\psi}):(V, \mathcal{B}) \rightarrow(U, \mathcal{A})$ is a morphism of quasi-coherent ringed schemes.

Lemma 1.5. Let $\left(X_{1}, \mathcal{A}_{1}\right)$ and $\left(X_{2}, \mathcal{A}_{2}\right)$ be quasi-coherent ringed schemes over $\mathbb{k}$, and assume a product $\left(X_{1} \times X_{2}, \mathcal{A}_{1} \otimes \mathcal{A}_{2}\right)$ exists. Then the projections $\mathrm{p}_{i}$ : $X_{1} \times X_{2} \rightarrow X_{i}$ extend to morphisms of ringed schemes

$$
\tilde{\mathrm{p}}_{i}:\left(X_{1} \times X_{2}, \mathcal{A}_{1} \otimes \mathcal{A}_{2}\right) \rightarrow\left(X_{i}, \mathcal{A}_{i}\right) .
$$

Proof. By symmetry it suffices to show that the first projection $\tilde{\mathrm{p}}_{1}$ exists. Let us choose affine open sets $U_{1} \subset X_{1}$ and $U_{2} \subset X_{2}$. By Lemma 1.4 the ring homomorphism

$$
\Gamma\left(U_{1}, \mathcal{A}_{1}\right) \rightarrow \Gamma\left(U_{1}, \mathcal{A}_{1}\right) \otimes \Gamma\left(U_{2}, \mathcal{A}_{2}\right) \cong \Gamma\left(U_{1} \times U_{2}, \mathcal{A}_{1} \otimes \mathcal{A}_{2}\right)
$$

extends to a morphism of ringed schemes

$$
\left.\tilde{\mathrm{p}}_{1}\right|_{U_{1} \times U_{2}}:\left(U_{1} \times U_{2},\left.\left(\mathcal{A}_{1} \otimes \mathcal{A}_{2}\right)\right|_{U_{1} \times U_{2}}\right) \rightarrow\left(U_{1},\left.\mathcal{A}_{1}\right|_{U_{1}}\right) \rightarrow\left(X_{1}, \mathcal{A}_{1}\right) .
$$

Because the morphisms $\left.\tilde{\mathrm{p}}_{1}\right|_{U_{1} \times U_{2}}$ depend functorially on the affine open rectangles $U_{1} \times U_{2}$ we can glue them to obtain a global morphism $\tilde{\mathrm{p}}_{1}$.

Proposition 1.6. Let $\left(X_{1}, \mathcal{A}_{1}\right),\left(X_{2}, \mathcal{A}_{2}\right),\left(Y_{1}, \mathcal{B}_{1}\right)$ and $\left(Y_{2}, \mathcal{B}_{2}\right)$ be quasi-coherent ringed schemes over $\mathbb{k}$, and let $\tilde{f}_{i}:\left(Y_{i}, \mathcal{B}_{i}\right) \rightarrow\left(X_{i}, \mathcal{A}_{i}\right)$ be morphisms. Assume products $\left(X_{1} \times X_{2}, \mathcal{A}_{1} \otimes \mathcal{A}_{2}\right)$ and $\left(Y_{1} \times Y_{2}, \mathcal{B}_{1} \otimes \mathcal{B}_{2}\right)$ exist. Then there is a unique morphism

$$
\tilde{f}_{1} \times \tilde{f}_{2}:\left(Y_{1} \times Y_{2}, \mathcal{B}_{1} \otimes \mathcal{B}_{2}\right) \rightarrow\left(X_{1} \times X_{2}, \mathcal{A}_{1} \otimes \mathcal{A}_{2}\right)
$$

such that $\tilde{\mathrm{p}}_{i} \circ\left(\tilde{f}_{1} \times \tilde{f}_{2}\right)=\tilde{f}_{i} \circ \tilde{\mathrm{p}}_{i}$.

Proof. Let $U_{i} \subset X_{i}$ and $V_{i} \subset f_{1}^{-1}\left(U_{i}\right)$ be affine open sets. The ring homomorphisms $\tilde{f}_{i}^{*}: \Gamma\left(U_{i}, \mathcal{A}_{i}\right) \rightarrow \Gamma\left(V_{i}, \mathcal{B}_{i}\right)$ give rise to a ring homomorphism

$$
\begin{aligned}
& \Gamma\left(U_{1} \times U_{2}, \mathcal{A}_{1} \otimes \mathcal{A}_{2}\right) \cong \Gamma\left(U_{1}, \mathcal{A}_{1}\right) \otimes \Gamma\left(U_{2}, \mathcal{A}_{2}\right) \\
& \quad \stackrel{\tilde{f}_{1}^{*} \otimes \tilde{f}_{2}^{*}}{\longrightarrow} \Gamma\left(V_{1}, \mathcal{B}_{1}\right) \otimes \Gamma\left(V_{2}, \mathcal{B}_{2}\right) \cong \Gamma\left(V_{1} \times V_{2}, \mathcal{B}_{1} \otimes \mathcal{B}_{2}\right) .
\end{aligned}
$$

Using Lemma 1.4 we obtain a unique morphism of ringed schemes

$$
\left(V_{1} \times V_{2},\left.\left(\mathcal{B}_{1} \otimes \mathcal{B}_{2}\right)\right|_{V_{1} \times V_{2}}\right) \rightarrow\left(U_{1} \times U_{2},\left.\left(\mathcal{A}_{1} \otimes \mathcal{A}_{2}\right)\right|_{U_{1} \times U_{2}}\right)
$$

that's compatible with the projections. By gluing we obtain $\tilde{f}_{1} \times \tilde{f}_{2}$.

Corollary 1.7. The product $\left(X_{1} \times X_{2}, \mathcal{A}_{1} \otimes \mathcal{A}_{2}\right)$, together with the projections $\tilde{\mathrm{p}}_{1}$ and $\tilde{\mathrm{p}}_{1}$, is unique up to a unique isomorphism.

Proof. Take $\left(X_{i}, \mathcal{A}_{i}\right)=\left(Y_{i}, \mathcal{B}_{i}\right)$ in Proposition 1.6

Observe that given a quasi-coherent $\mathcal{O}_{X}$-ring $\mathcal{A}$ the opposite ring $\mathcal{A}^{\mathrm{op}}$ is also a quasi-coherent $\mathcal{O}_{X}$-ring. 
Definition 1.8. Let $(X, \mathcal{A})$ be a quasi-coherent ringed scheme over $\mathbb{k}$. We denote by

$$
\left(X^{2}, \mathcal{A}^{\mathrm{e}}\right):=\left(X \times X, \mathcal{A} \otimes \mathcal{A}^{\mathrm{op}}\right),
$$

the product of the quasi-coherent ringed schemes $(X, \mathcal{A})$ and $\left(X, \mathcal{A}^{\mathrm{op}}\right)$.

Here is an easy example of a product.

Example 1.9. Let $(X, \mathcal{A})$ be any quasi-coherent ringed scheme. Let $B$ be a $\mathbb{k}$ algebra, which we consider as a quasi-coherent ringed scheme $(Y, \mathcal{B}):=(\operatorname{Spec} \mathbb{k}, B)$. Then the product exists, and it is

$$
(X \times Y, \mathcal{A} \otimes \mathcal{B})=(X, \mathcal{A} \otimes B) .
$$

The existence of products turns out to be more complicated in general, as we see in the next theorem.

Theorem 1.10. Let $(X, \mathcal{A})$ and $(Y, \mathcal{B})$ be quasi-coherent ringed schemes over $\mathbb{k}$. The two conditions below are equivalent.

(i) The product $(X \times Y, \mathcal{A} \otimes \mathcal{B})$ exists.

(ii) Given any pair of affine open sets $U \subset X$ and $V \subset Y$, write $C:=$ $\Gamma\left(U \times V, \mathcal{O}_{X \times Y}\right), A:=\Gamma(U, \mathcal{A})$ and $B:=\Gamma(V, \mathcal{B})$. Let $S \subset C$ be any multiplicatively closed set. Then the image $\bar{S}$ of $S$ in $A \otimes B$ is a denominator set.

Proof. (i) $\Rightarrow$ (ii): Choose affine open sets $U \subset X$ and $V \subset Y$. Then $\left.(\mathcal{A} \otimes \mathcal{B})\right|_{U \times V}$ is a quasi-coherent $\mathcal{O}_{U \times V}$-ring satisfying

$$
\Gamma\left(U \times V,\left.(\mathcal{A} \otimes \mathcal{B})\right|_{U \times V}\right) \cong A \otimes B
$$

as $C$-rings. According to [YZ4 Corollary 5.13] for any multiplicatively closed set $S \subset C$ the set $\bar{S} \subset A \otimes B$ is a denominator set.

(ii) $\Rightarrow$ (i): Let $U \subset X$ and $V \subset Y$ be arbitrary affine open sets. According to YZ4, Corollary 5.13] there is a quasi-coherent $\mathcal{O}_{U \times V}$-ring, call it $\left.\left.\mathcal{A}\right|_{U} \otimes \mathcal{B}\right|_{V}$, such that

$$
\Gamma\left(U \times V,\left.\left.\mathcal{A}\right|_{U} \otimes \mathcal{B}\right|_{V}\right) \cong A \otimes B
$$

as $C$-rings. We claim that $\left(U \times V,\left.\left.\mathcal{A}\right|_{U} \otimes \mathcal{B}\right|_{V}\right)$ is a product of $\left(U,\left.\mathcal{A}\right|_{U}\right)$ and $\left(V,\left.\mathcal{B}\right|_{V}\right)$.

By Lemma 1.4 the ring homomorphism $A \rightarrow A \otimes B$ induces a morphism of ringed schemes $\left(U \times V,\left.\left.\mathcal{A}\right|_{U} \otimes \mathcal{B}\right|_{V}\right) \rightarrow\left(U,\left.\mathcal{A}\right|_{U}\right)$, which in turn gives us a homomorphism of sheaves of rings $\left.\left.\left.\left(\mathrm{p}_{1}^{-1} \mathcal{A}\right)\right|_{U \times V} \rightarrow \mathcal{A}\right|_{U} \otimes \mathcal{B}\right|_{V}$. Likewise we get $\left.\left(\mathrm{p}_{2}^{-1} \mathcal{B}\right)\right|_{U \times V} \rightarrow$ $\left.\left.\mathcal{A}\right|_{U} \otimes \mathcal{B}\right|_{V}$. Multiplying inside $\left.\left.\mathcal{A}\right|_{U} \otimes \mathcal{B}\right|_{V}$ we obtain a homomorphism of modules

$$
\left.\phi\right|_{U \times V}:\left.\left.\left.\left.\left(\mathrm{p}_{1}^{-1} \mathcal{A}\right)\right|_{U \times V} \otimes\left(\mathrm{p}_{2}^{-1} \mathcal{B}\right)\right|_{U \times V} \rightarrow \mathcal{A}\right|_{U} \otimes \mathcal{B}\right|_{V} .
$$

In order to show that this is a homomorphism of sheaves of rings it suffices to check that for any point $z \in U \times V$ the images of $\left.\left(\mathrm{p}_{1}^{-1} \mathcal{A}\right)\right|_{U \times V}$ and $\left.\left(\mathrm{p}_{2}^{-1} \mathcal{B}\right)\right|_{U \times V}$ inside the stalk $\left(\left.\left.\mathcal{A}\right|_{U} \otimes \mathcal{B}\right|_{V}\right)_{z}$ commute. Let us write $x:=\mathrm{p}_{1}(z)$ and $y:=\mathrm{p}_{2}(z)$. Then $\left(\mathrm{p}_{1}^{-1} \mathcal{A}\right)_{z} \cong \mathcal{A}_{x}$ and $\left(\mathrm{p}_{2}^{-1} \mathcal{B}\right)_{z} \cong \mathcal{B}_{y}$. As explained in the proof of Lemma 2.1 the ring $\left(\left.\left.\mathcal{A}\right|_{U} \otimes \mathcal{B}\right|_{V}\right)_{z}$ is an Ore localization of $\mathcal{A}_{x} \otimes \mathcal{B}_{y}$, so indeed there is commutation.

The formation of the homomorphism $\left.\phi\right|_{U \times V}$ is functorial in the affine open rectangle $U \times V$, and hence we can glue. By construction conditions (i)-(ii) of Definition 1.3 are satisfied.

Example 1.11. If $\mathcal{A}$ is an $\mathcal{O}_{X}$-algebra (i.e. the image of $\mathcal{O}_{X}$ is in the center $\mathrm{Z}(\mathcal{A})$ )

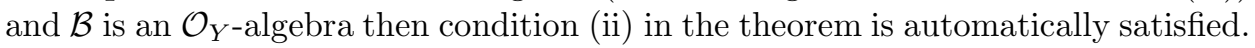
Hence the product $(X \times Y, \mathcal{A} \otimes \mathcal{B})$ exists. 
Remark 1.12. Our definition of quasi-coherent ringed scheme is slightly different than that of Lunts $\mathrm{Lu}$. While in $\mathrm{Lu}$ the existence of a product must be stipulated, here we have the criterion in Theorem[1.10] There are even counterexamples; indeed in [Y4, Example 5.14] we exhibit an affine quasi-coherent ringed scheme $(X, \mathcal{A})$ for which the product $\left(X^{2}, \mathcal{A}^{\mathrm{e}}\right)$ does not exist.

Let $(X, \mathcal{A})$ be a quasi-coherent ringed scheme over $\mathbb{k}$. We denote by $\operatorname{Mod} \mathcal{A}$ the category of left $\mathcal{A}$-modules, and by $\mathrm{D}(\operatorname{Mod} \mathcal{A})$ its derived category. The category $\operatorname{Mod} \mathcal{A}$ is abelian and it has enough injectives. Given an injective $\mathcal{A}$-module $\mathcal{I}$ its restriction $\left.\mathcal{I}\right|_{U}$ to an open subset $U$ is an injective $\left.\mathcal{A}\right|_{U}$-module. Any complex $\mathcal{N} \in \mathrm{D}^{+}(\operatorname{Mod} \mathcal{A})$ has an injective resolution $\mathcal{N} \rightarrow \mathcal{I}$, namely a quasi-isomorphism to a bounded below complex $\mathcal{I}$ of injective $\mathcal{A}$-modules. This allows us to define the derived functor

$$
\operatorname{RHom}_{\mathcal{A}}(-,-): \mathrm{D}^{-}(\operatorname{Mod} \mathcal{A})^{\mathrm{op}} \times \mathrm{D}^{+}(\operatorname{Mod} \mathcal{A}) \rightarrow \mathrm{D}^{+}\left(\operatorname{Mod}_{X}\right),
$$

where $\mathbb{k}_{X}$ is the constant sheaf $\mathbb{k}$ on $X$. The formula is $\operatorname{RHom} m_{\mathcal{A}}(\mathcal{M}, \mathcal{N}):=$ $\mathcal{H o m}_{\mathcal{A}}(\mathcal{M}, \mathcal{I})$ for any injective resolution $\mathcal{N} \rightarrow \mathcal{I}$. Since $\mathcal{H o m}_{\mathcal{A}}(\mathcal{M}, \mathcal{I})$ is a bounded below complex of flasque sheaves it follows that

$$
\operatorname{RHom}_{\mathcal{A}}(\mathcal{M}, \mathcal{N}) \cong \mathrm{R} \Gamma\left(X, \operatorname{RHom}_{\mathcal{A}}(\mathcal{M}, \mathcal{N})\right)
$$

which is a functor

$$
\operatorname{RHom}_{\mathcal{A}}(-,-): \mathrm{D}^{-}(\operatorname{Mod} \mathcal{A})^{\mathrm{op}} \times \mathrm{D}^{+}(\operatorname{Mod} \mathcal{A}) \rightarrow \mathrm{D}^{+}(\operatorname{Mod} \mathbb{k}) .
$$

For more details regarding derived categories of sheaves see [RD, [KS] or [Bor].

Remark 1.13. One can of course remove some boundedness restrictions using Kinjective resolutions, but we are not going to worry about this. Already there are enough delicate issues regarding injective resolutions of quasi-coherent $\mathcal{A}$-modules; see Remark 1.16.

Definition 1.14. Let $(X, \mathcal{A})$ be a quasi-coherent ringed scheme over $\mathbb{k}$. Suppose $X$ is noetherian, and for every affine open set $U \subset X$ the $\operatorname{ring} \Gamma(U, \mathcal{A})$ is left noetherian. Then we call $\mathcal{A}$ a left noetherian quasi-coherent $\mathcal{O}_{X}$-ring, and the pair $(X, \mathcal{A})$ is called a left noetherian quasi-coherent ringed scheme. We say $\mathcal{A}$ is right noetherian if $\mathcal{A}^{\mathrm{op}}$ is left noetherian. If $\mathcal{A}$ is both left and right noetherian then the pair $(X, \mathcal{A})$ is called a noetherian quasi-coherent ringed scheme.

When we speak of a left or right noetherian quasi-coherent $\mathcal{O}_{X}$-ring $\mathcal{A}$ we tacitly assume that $X$ itself is noetherian.

An $\mathcal{A}$-module $\mathcal{M}$ is called quasi-coherent if locally, on every sufficiently small open set $U$, the $\left.\mathcal{A}\right|_{U}$-module $\left.\mathcal{M}\right|_{U}$ has a free presentation (i.e. it is the cokernel of a homomorphism between free $\left.\mathcal{A}\right|_{U}$-modules); cf. [EGA I]. Equivalently, $\mathcal{M}$ is quasicoherent as $\mathcal{O}_{X}$-module. If $\mathcal{A}$ is left noetherian then $\mathcal{M}$ is a coherent $\mathcal{A}$-module if and only if it is quasi-coherent and locally finitely generated. We shall denote the categories of quasi-coherent (resp. coherent) $\mathcal{A}$-modules by QCoh $\mathcal{A}$ (resp. Coh $\mathcal{A}$ ). For a ring $A$ we write $\operatorname{Mod}_{\mathrm{f}} A$ for the category of finite (i.e. finitely generated) modules.

Proposition 1.15. Let $(X, \mathcal{A})$ be a quasi-coherent ringed scheme, let $U \subset X$ be an affine open set and $A:=\Gamma(U, \mathcal{A})$.

(1) The functor $\Gamma(U,-)$ is an equivalence of categories $\left.\mathrm{QCoh} \mathcal{A}\right|_{U} \rightarrow \operatorname{Mod} A$. 
(2) If $\mathcal{A}$ is left noetherian then $\Gamma(U,-)$ restricts to an equivalence of categories $\left.\operatorname{Coh} \mathcal{A}\right|_{U} \rightarrow \operatorname{Mod}_{\mathrm{f}} A$.

Proof. This is a slight generalization of EGA I, Corollary 1.4.2 and Theorem 1.5.1]. See also [Ha, Corollary II.5.5].

In the context of the proposition above, given an $A$-module $M$ we shall usually denote the corresponding quasi-coherent $\left.\mathcal{A}\right|_{U}$-module by $\left.\mathcal{A}\right|_{U} \otimes_{A} M$.

Remark 1.16. By [Ka, Remark 3.2] any injective object $\mathcal{I}$ in QCoh $\mathcal{A}$ is a flasque sheaf on $X$. However $\mathcal{I}$ might not be injective in the bigger category $\operatorname{Mod} \mathcal{A}$, and there are counterexamples (see $\mathrm{Ka}$ Remark 7.4]). Moreover, we do not know if the restriction to an open set $\left.\mathcal{I}\right|_{U}$ is injective in QCoh $\left.\mathcal{A}\right|_{U}$; the results of [GJ] seem to indicate otherwise. This is in contrast to the commutative noetherian case $\mathcal{A}=\mathcal{O}_{X}$, in which $\left.\mathcal{I}\right|_{U}$ is always injective in $\left.\operatorname{Mod} \mathcal{A}\right|_{U}$; see $[\mathrm{RD}$, Proposition II.7.17].

As is customary we denote by $\mathrm{D}_{\mathrm{qc}}(\operatorname{Mod} \mathcal{A})\left(\operatorname{resp} . \mathrm{D}_{\mathrm{c}}(\operatorname{Mod} \mathcal{A})\right)$ the full subcategory of $\mathrm{D}(\operatorname{Mod} \mathcal{A})$ consisting of complexes with quasi-coherent (resp. coherent) cohomology modules. These are triangulated subcategories (the latter if $\mathcal{A}$ is left noetherian).

We now recall a theorem of Bernstein about equivalences of derived categories of $\mathcal{A}$-modules.

Theorem 1.17 (B⿺r, Theorem VI.2.10 and Proposition VI.2.11]). Let $(X, \mathcal{A})$ be a separated quasi-coherent ringed scheme.

(1) The inclusion functor $\mathrm{D}^{\mathrm{b}}(\mathrm{Q} \operatorname{Coh} \mathcal{A}) \rightarrow \mathrm{D}_{\mathrm{qc}}^{\mathrm{b}}(\operatorname{Mod} \mathcal{A})$ is an equivalence.

(2) If in addition $\mathcal{A}$ is left noetherian then the inclusion functor $\mathrm{D}^{\mathrm{b}}(\operatorname{Coh} \mathcal{A}) \rightarrow$ $\mathrm{D}_{\mathrm{c}}^{\mathrm{b}}(\operatorname{Mod} \mathcal{A})$ is an equivalence.

Corollary 1.18. Assume $(X, \mathcal{A})$ is a quasi-coherent ringed scheme and $U \subset X$ is an affine open set. Write $A:=\Gamma(U, \mathcal{A})$. Then

$$
\mathrm{R} \Gamma(U,-): \mathrm{D}_{\mathrm{qc}}^{\mathrm{b}}\left(\left.\operatorname{Mod} \mathcal{A}\right|_{U}\right) \rightarrow \mathrm{D}^{\mathrm{b}}(\operatorname{Mod} A)
$$

is an equivalence with inverse $\left.M \mapsto \mathcal{A}\right|_{U} \otimes_{A} M$. If $\mathcal{A}$ is left noetherian then we get an equivalence

$$
\mathrm{R} \Gamma(U,-): \mathrm{D}_{\mathrm{c}}^{\mathrm{b}}\left(\left.\operatorname{Mod} \mathcal{A}\right|_{U}\right) \rightarrow \mathrm{D}_{\mathrm{f}}^{\mathrm{b}}(\operatorname{Mod} A) .
$$

Proof. By Proposition 1.15 any quasi-coherent $\mathcal{O}_{U}$-module is acyclic for the functor $\Gamma(U,-)$. Hence if $\mathcal{M} \in \mathrm{D}^{\mathrm{b}}\left(\left.\mathrm{Q} \operatorname{Coh} \mathcal{A}\right|_{U}\right)$, and if $\mathcal{M} \rightarrow \mathcal{I}$ is a quasi-isomorphism with $\mathcal{I}$ a bounded below complex of injective $\left.\mathcal{A}\right|_{U}$-modules (not necessarily quasicoherent), then $\Gamma(U, \mathcal{M}) \rightarrow \Gamma(U, \mathcal{I})$ is a quasi-isomorphism. It follows that the composed functor

$$
\mathrm{D}^{\mathrm{b}}\left(\left.\mathrm{Q} \operatorname{Coh} \mathcal{A}\right|_{U}\right) \rightarrow \mathrm{D}_{\mathrm{qc}}^{\mathrm{b}}\left(\left.\operatorname{Mod} \mathcal{A}\right|_{U}\right) \stackrel{\mathrm{R} \Gamma(U,-)}{\longrightarrow} \mathrm{D}^{\mathrm{b}}(\operatorname{Mod} A)
$$

is an equivalence. Now use Theorem 1.17

Lemma 1.19. Let $(X, \mathcal{A})$ be a quasi-coherent ringed scheme and let $V \subset U$ be two affine open sets. Let $A:=\Gamma(U, \mathcal{A})$ and $A^{\prime}:=\Gamma(V, \mathcal{A})$. Then the diagram

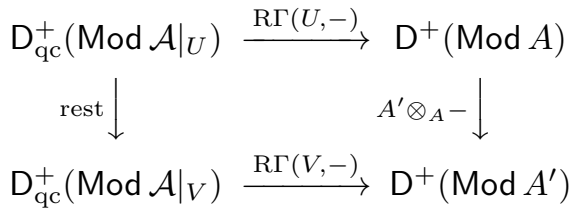


is commutative.

Proof. First we note that $A^{\prime}$ is a flat $A$-module on both sides (see YZ4, Proposition 5.6]. Given $\mathcal{M} \in \mathrm{D}_{\mathrm{qc}}^{+}\left(\left.\operatorname{Mod} \mathcal{A}\right|_{U}\right)$ take a resolution $\mathcal{M} \rightarrow \mathcal{I}$ where $\mathcal{I}$ is a bounded below complex of injective $\left.\mathcal{A}\right|_{U}$-modules. Then $\left.\left.\mathcal{M}\right|_{V} \rightarrow \mathcal{I}\right|_{V}$ is an injective resolution. We get a natural morphism

$$
\mathrm{R} \Gamma(U, \mathcal{M})=\Gamma(U, \mathcal{I}) \rightarrow \Gamma(V, \mathcal{I})=\mathrm{R} \Gamma(V, \mathcal{M}),
$$

and hence a morphism

$$
A^{\prime} \otimes_{A} \mathrm{R} \Gamma(U, \mathcal{M}) \rightarrow \mathrm{R} \Gamma(V, \mathcal{M}) .
$$

To show the latter is an isomorphism it suffices to check for a single quasi-coherent $\left.\mathcal{A}\right|_{U}$-module $\mathcal{M}$ - since these are way-out functors, cf. [RD Section I.7]. But for such $\mathcal{M}$ we have $\mathrm{R} \Gamma(U, \mathcal{M})=\Gamma(U, \mathcal{M})$ and $\mathrm{R} \Gamma(V, \mathcal{M})=\Gamma(V, \mathcal{M})$, so $\mathrm{YZ4}$, Proposition 5.6] applies.

\section{Dualizing Complexes over Ringed Schemes}

Derived functors in the noncommutative situation are inherently more complicated than in the commutative situation. Therefore we begin this section with some technical results that are needed to state our definition of dualizing complexes over quasi-coherent ringed schemes.

Lemma 2.1. Let $(X, \mathcal{A})$ and $(Y, \mathcal{B})$ be two quasi-coherent ringed schemes over $\mathbb{k}$. Assume the product $(X \times Y, \mathcal{A} \otimes \mathcal{B})$ exists. Then:

(1) The homomorphisms of sheaves of rings

$$
\mathrm{p}_{1}^{-1} \mathcal{A} \rightarrow\left(\mathrm{p}_{1}^{-1} \mathcal{A}\right) \otimes\left(\mathrm{p}_{2}^{-1} \mathcal{B}\right) \rightarrow \mathcal{A} \otimes \mathcal{B}
$$

are flat on both sides.

(2) An injective $\mathcal{A} \otimes \mathcal{B}$-module is also an injective $\mathrm{p}_{1}^{-1} \mathcal{A}$-module.

(3) Given a $\mathrm{p}_{1}^{-1} \mathcal{A}$-module $\mathcal{M}$ and an injective $\mathcal{A} \otimes \mathcal{B}$-module $\mathcal{I}$, the $\mathrm{p}_{2}^{-1} \mathcal{B}$ - module $\mathcal{H o m}_{\mathrm{p}_{1}^{-1} \mathcal{A}}(\mathcal{M}, \mathcal{I})$ is injective.

These statements hold also if we exchange $\mathrm{p}_{1}^{-1} \mathcal{A}$ with $\mathrm{p}_{2}^{-1} \mathcal{B}$, or $\mathrm{p}_{1}^{-1} \mathcal{A}$ with $\mathrm{p}_{1}^{-1} \mathcal{A}^{\mathrm{op}}$, etc.

Proof. (1) Flatness can be checked on stalks at points of $X \times Y$. Take an arbitrary point $z \in X \times Y$, and let $x:=\mathrm{p}_{1}(z) \in X$ and $y:=\mathrm{p}_{2}(z) \in Y$. The stalks satisfy $\left(\mathrm{p}_{1}^{-1} \mathcal{A}\right)_{z} \cong \mathcal{A}_{x},\left(\mathrm{p}_{2}^{-1} \mathcal{B}\right)_{z} \cong \mathcal{B}_{y}$ and

$$
\left(\left(\mathrm{p}_{1}^{-1} \mathcal{A}\right) \otimes\left(\mathrm{p}_{2}^{-1} \mathcal{B}\right)\right)_{z} \cong\left(\mathrm{p}_{1}^{-1} \mathcal{A}\right)_{z} \otimes\left(\mathrm{p}_{2}^{-1} \mathcal{B}\right)_{z} \cong \mathcal{A}_{x} \otimes \mathcal{B}_{y}
$$

Hence the homomorphism

$$
\left(\mathrm{p}_{1}^{-1} \mathcal{A}\right)_{z} \rightarrow\left(\left(\mathrm{p}_{1}^{-1} \mathcal{A}\right) \otimes\left(\mathrm{p}_{2}^{-1} \mathcal{B}\right)\right)_{z}
$$

is flat on both sides.

Choose affine open sets $U \subset X$ and $V \subset Y$ such that $z \in U \times V$. By definition

$$
\Gamma(U \times V, \mathcal{A} \otimes \mathcal{B}) \cong \Gamma(U, \mathcal{A}) \otimes \Gamma(V, \mathcal{B}) .
$$

Now the stalks $\mathcal{A}_{x}, \mathcal{B}_{y}$ and $(\mathcal{A} \otimes \mathcal{B})_{z}$ are Ore localizations of $\Gamma(U, \mathcal{A}), \Gamma(V, \mathcal{B})$ and $\Gamma(U \times V, \mathcal{A} \otimes \mathcal{B})$ respectively, for suitable denominator sets. Hence $(\mathcal{A} \otimes \mathcal{B})_{z}$ is an Ore localization of $\left(\mathcal{A}_{x} \otimes \mathcal{B}_{y}\right)$, so it's flat. 
(2) By part (1) the functor $\mathcal{M} \mapsto(\mathcal{A} \otimes \mathcal{B}) \otimes_{\mathrm{p}_{1}^{-1} \mathcal{A}} \mathcal{M}$ from $\operatorname{Mod} \mathrm{p}_{1}^{-1} \mathcal{A}$ to $\operatorname{Mod} \mathcal{A} \otimes \mathcal{B}$ is exact. Hence for an injective $\mathcal{A} \otimes \mathcal{B}$-module $\mathcal{I}$ the functor

$$
\mathcal{M} \mapsto \operatorname{Hom}_{\mathrm{p}_{1}^{-1} \mathcal{A}}(\mathcal{M}, \mathcal{I}) \cong \operatorname{Hom}_{\mathcal{A} \otimes \mathcal{B}}\left((\mathcal{A} \otimes \mathcal{B}) \otimes_{\mathrm{p}_{1}^{-1} \mathcal{A}} \mathcal{M}, \mathcal{I}\right)
$$

is exact, implying that $\mathcal{I}$ is an injective $\mathrm{p}_{1}^{-1} \mathcal{A}$-module.

(3) Similar to (2).

Lemma 2.2. Let $(X, \mathcal{A})$ and $(Y, \mathcal{B})$ be quasi-coherent ringed schemes over $\mathbb{k}$. Assume that the product $\left(X \times Y, \mathcal{A} \otimes \mathcal{B}^{\mathrm{op}}\right)$ exists. Let $\mathcal{R} \in \mathrm{D}^{+}\left(\operatorname{Mod} \mathcal{A} \otimes \mathcal{B}^{\mathrm{op}}\right)$ be some complex.

(1) There are functors

$$
\begin{gathered}
\mathrm{D}: \mathrm{D}^{\mathrm{b}}(\operatorname{Mod} \mathcal{A})^{\mathrm{op}} \rightarrow \mathrm{D}\left(\operatorname{Mod} \mathcal{B}^{\mathrm{op}}\right), \\
\mathrm{D}^{\mathrm{op}}: \mathrm{D}^{\mathrm{b}}\left(\operatorname{Mod} \mathcal{B}^{\mathrm{op}}\right)^{\mathrm{op}} \rightarrow \mathrm{D}(\operatorname{Mod} \mathcal{A})
\end{gathered}
$$

defined by the formulas

$$
\begin{aligned}
\mathrm{D} \mathcal{M} & :=\mathrm{Rp}_{2 *} \mathrm{RH} m_{\mathrm{p}_{1}^{-1} \mathcal{A}}\left(\mathrm{p}_{1}^{-1} \mathcal{M}, \mathcal{R}\right), \\
\mathrm{D}^{\mathrm{op}} \mathcal{N}: & :=\mathrm{Rp}_{1 *} \mathrm{RHom}_{\mathrm{p}_{2}^{-1} \mathcal{B}_{\mathrm{op}}}\left(\mathrm{p}_{2}^{-1} \mathcal{N}, \mathcal{R}\right) .
\end{aligned}
$$

(2) If $\mathrm{H}^{i} \mathrm{D} \mathcal{M}=0$ for $i \gg 0$ then $\mathrm{D}^{\mathrm{op}} \mathrm{D} \mathcal{M}$ is well defined, and there is a morphism $\mathcal{M} \rightarrow \mathrm{D}^{\mathrm{op}} \mathrm{D} \mathcal{M}$ in $\mathrm{D}(\operatorname{Mod} \mathcal{A})$, which is functorial in $\mathcal{M}$. Similarly for $\mathcal{N}$ and $\mathrm{DD}^{\mathrm{op}} \mathcal{N}$.

Proof. (1) Choose a quasi-isomorphism $\mathcal{R} \rightarrow \mathcal{I}$ with $\mathcal{I}$ a bounded below complex of injective $\mathcal{A} \otimes \mathcal{B}^{\text {op }}$-modules. By Lemma 2.1](2) $\mathcal{I}$ is a complex of injective $\mathrm{p}_{1}^{-1} \mathcal{A}$ -modules, and we get

$$
\mathrm{RH}^{\circ} m_{\mathrm{p}_{1}^{-1} \mathcal{A}}\left(\mathrm{p}_{1}^{-1} \mathcal{M}, \mathcal{R}\right)=\mathcal{H}^{\prime} m_{\mathrm{p}_{1}^{-1} \mathcal{A}}\left(\mathrm{p}_{1}^{-1} \mathcal{M}, \mathcal{I}\right) \in \mathrm{D}\left(\operatorname{Mod~p}_{2}^{-1} \mathcal{B}^{\mathrm{op}}\right) .
$$

Because $\mathcal{H o m} \mathrm{p}_{1}^{-1} \mathcal{A}\left(\mathrm{p}_{1}^{-1} \mathcal{M}, \mathcal{I}\right)$ is a bounded below complex of injective $\mathrm{p}_{2}^{-1} \mathcal{B}^{\text {op }}$ modules (see Lemma 2.1(3)) we get

$$
\operatorname{Rp}_{2 *} \mathrm{RH}_{\mathcal{H}} m_{\mathrm{p}_{1}^{-1} \mathcal{A}}\left(\mathrm{p}_{1}^{-1} \mathcal{M}, \mathcal{R}\right)=\mathrm{p}_{2 *} \mathcal{H} o m_{\mathrm{p}_{1}^{-1} \mathcal{A}}\left(\mathrm{p}_{1}^{-1} \mathcal{M}, \mathcal{I}\right) \in \mathrm{D}\left(\operatorname{Mod} \mathcal{B}^{\mathrm{op}}\right)
$$

Likewise for $\mathrm{D}^{\mathrm{op}}$.

(2) Let $\mathcal{R} \rightarrow \mathcal{I}$ be as above. If $\mathrm{H}^{i} \mathrm{D} \mathcal{M}=0$ for $i \gg 0$ then $\mathrm{D} \mathcal{M} \cong \mathcal{L}$ in $\mathrm{D}\left(\operatorname{Mod} \mathcal{B}^{\text {op }}\right)$, where $\mathcal{L} \in \mathrm{D}^{\mathrm{b}}\left(\operatorname{Mod} \mathcal{B}^{\mathrm{op}}\right)$ is a truncation of $\mathrm{D} \mathcal{M}$. So $\mathrm{D}^{\mathrm{op}} \mathrm{D} \mathcal{M}:=\mathrm{D}^{\mathrm{op}} \mathcal{L}$ is well defined. Adjunction gives a morphism

$$
\mathrm{p}_{2}^{-1} \mathcal{L} \cong \mathrm{p}_{2}^{-1} \mathrm{p}_{2 *} \mathcal{H} o m_{\mathrm{p}_{1}^{-1} \mathcal{A}}\left(\mathrm{p}_{1}^{-1} \mathcal{M}, \mathcal{I}\right) \rightarrow \mathcal{H} o m_{\mathrm{p}_{1}^{-1} \mathcal{A}}\left(\mathrm{p}_{1}^{-1} \mathcal{M}, \mathcal{I}\right)
$$

in $\mathrm{D}\left(\operatorname{Mod} \mathrm{p}_{1}^{-1} \mathcal{A}\right)$. Applying $\mathcal{H o m}_{\mathrm{p}_{2}^{-1} \mathcal{B}^{\mathrm{op}}}(-, \mathcal{I})$ and a second adjunction we get a morphism

$$
\mathrm{p}_{1}^{-1} \mathcal{M} \rightarrow \mathcal{H o m}_{\mathrm{p}_{2}^{-1} \mathcal{B}_{\mathrm{op}}}\left(\mathcal{H o m}_{\mathrm{p}_{1}^{-1} \mathcal{A}}\left(\mathrm{p}_{1}^{-1} \mathcal{M}, \mathcal{I}\right), \mathcal{I}\right) \rightarrow \mathcal{H} o m_{\mathrm{p}_{2}^{-1} \mathcal{B}^{\mathrm{op}}}\left(\mathrm{p}_{2}^{-1} \mathcal{L}, \mathcal{I}\right)
$$

Finally a third adjunction gives a morphism

$$
\mathcal{M} \rightarrow \mathrm{p}_{1 *} \mathrm{p}_{1}^{-1} \mathcal{M} \rightarrow \mathrm{p}_{1 *} \mathcal{H} \text { om } \mathrm{p}_{2}^{-1} \mathcal{B}_{\mathrm{op}}\left(\mathrm{p}_{2}^{-1} \mathcal{L}, \mathcal{I}\right)=\mathrm{D}^{\mathrm{op}} \mathcal{L}
$$

All morphisms occurring above (including the truncation $\mathrm{D} \mathcal{M} \cong \mathcal{L}$ ) are functorial in $\mathcal{M}$. 
Definition 2.3. Let $(X, \mathcal{A})$ and $(Y, \mathcal{B})$ be separated quasi-coherent ringed schemes over $\mathbb{k}$. Assume $\mathcal{A}$ and $\mathcal{B}^{\text {op }}$ are left noetherian, and the product $\left(X \times Y, \mathcal{A} \otimes \mathcal{B}^{\text {op }}\right)$ exists. A complex $\mathcal{R} \in \mathrm{D}_{\mathrm{qc}}^{\mathrm{b}}\left(\operatorname{Mod} \mathcal{A} \otimes \mathcal{B}^{\mathrm{op}}\right)$ is called a dualizing complex over $(\mathcal{A}, \mathcal{B})$ if conditions (i)-(iii) below hold for the functors $\mathrm{D}$ and $\mathrm{D}^{\text {op }}$ from Lemma 2.2 (1).

(i) The functors $\mathrm{D}$ and $\mathrm{D}^{\mathrm{op}}$ have finite cohomological dimensions when restricted to $\operatorname{Coh} \mathcal{A}$ and $\operatorname{Coh} \mathcal{B}^{\text {op }}$ respectively.

(ii) The functor $\mathrm{D}$ sends $\operatorname{Coh} \mathcal{A}$ into $\mathrm{D}_{\mathrm{c}}\left(\operatorname{Mod} \mathcal{B}^{\mathrm{op}}\right)$, and the functor $\mathrm{D}^{\mathrm{op}}$ sends $\operatorname{Coh} \mathcal{B}^{\text {op }}$ into $\mathrm{D}_{\mathrm{c}}(\operatorname{Mod} \mathcal{A})$.

(iii) The adjunction morphisms $\mathbf{1} \rightarrow \mathrm{D}^{\mathrm{op}} \mathrm{D}$ in $\mathrm{D}_{\mathrm{c}}^{\mathrm{b}}(\operatorname{Mod} \mathcal{A})$, and $\mathbf{1} \rightarrow \mathrm{DD}^{\mathrm{op}}$ in $\mathrm{D}_{\mathrm{c}}^{\mathrm{b}}\left(\operatorname{Mod} \mathcal{B}^{\mathrm{op}}\right)$, are both isomorphisms.

Note that conditions (i)-(ii) imply that there are well defined functors

$$
\begin{array}{r}
\mathrm{D}: \mathrm{D}_{\mathrm{c}}^{\mathrm{b}}(\operatorname{Mod} \mathcal{A})^{\mathrm{op}} \rightarrow \mathrm{D}_{\mathrm{c}}^{\mathrm{b}}\left(\operatorname{Mod} \mathcal{B}^{\mathrm{op}}\right), \\
\mathrm{D}^{\mathrm{op}}: \mathrm{D}_{\mathrm{c}}^{\mathrm{b}}\left(\operatorname{Mod} \mathcal{B}^{\mathrm{op}}\right)^{\mathrm{op}} \rightarrow \mathrm{D}_{\mathrm{c}}^{\mathrm{b}}(\operatorname{Mod} \mathcal{A}) .
\end{array}
$$

By Lemma 2.2 2) we have the adjunction morphisms appearing in condition (iii).

Convention 2.4. When we talk about a dualizing complex $\mathcal{R}$ over $(\mathcal{A}, \mathcal{B})$ we tacitly assume that we are given separated quasi-coherent ringed $\mathbb{k}$-schemes $(X, \mathcal{A})$ and $(Y, \mathcal{B})$, that $\mathcal{A}$ and $\mathcal{B}^{\text {op }}$ are left noetherian, and that the product $\left(X \times Y, \mathcal{A} \otimes \mathcal{B}^{\text {op }}\right)$ exists.

Definition 2.5. Let $(X, \mathcal{A})$ be a separated noetherian quasi-coherent ringed scheme over $\mathbb{k}$. Suppose the product $\left(X^{2}, \mathcal{A}^{\mathrm{e}}\right)=\left(X \times X, \mathcal{A} \otimes \mathcal{A}^{\mathrm{op}}\right)$ exists. If $\mathcal{R} \in$ $\mathrm{D}_{\mathrm{qc}}^{\mathrm{b}}\left(\operatorname{Mod} \mathcal{A}^{\mathrm{e}}\right)$ is a dualizing complex over $(\mathcal{A}, \mathcal{A})$ then we say $\mathcal{R}$ is a dualizing complex over $\mathcal{A}$.

Example 2.6. Suppose $X$ is a separated finite type $\mathbb{k}$-scheme and $\mathcal{R}$ is a dualizing complex over $X$ in the sense of $\left[\mathrm{RD}\right.$. Let $\Delta: X \rightarrow X^{2}$ be the diagonal embedding. Then $\Delta_{*} \mathcal{R}$ is a dualizing complex over $\mathcal{O}_{X}$ in the noncommutative sense, i.e. in the sense of Definition 2.5

Let us now recall the definition of dualizing complex over a pair of rings from Ye1] and YZ2].

Definition 2.7. Let $A$ be a left noetherian $\mathbb{k}$-algebra and $B$ a right noetherian $\mathbb{k}$-algebra. A complex $R \in \mathrm{D}^{\mathrm{b}}\left(\operatorname{Mod} A \otimes B^{\mathrm{op}}\right)$ is called a dualizing complex over $(A, B)$ if it satisfies the following three conditions:

(i) $R$ has finite injective dimension over $A$ and over $B^{\text {op }}$.

(ii) $R$ has finitely generated cohomology modules over $A$ and over $B^{\text {op }}$.

(iii) The canonical morphisms $B \rightarrow \operatorname{RHom}_{A}(R, R)$ in $\mathrm{D}\left(\operatorname{Mod} B^{\mathrm{e}}\right)$, and $A \rightarrow$ $\mathrm{RHom}_{B^{\text {op }}}(R, R)$ in $\mathrm{D}\left(\operatorname{Mod} A^{\mathrm{e}}\right)$, are both isomorphisms.

In the case $A=B$ we say $R$ is a dualizing complex over $A$.

Example 2.8. In the situation of Definition 2.7 above let $R \in \mathrm{D}^{\mathrm{b}}\left(\operatorname{Mod} A \otimes B^{\mathrm{op}}\right)$. Consider the quasi-coherent ringed schemes $(\operatorname{Spec} k, A)$ and $(\operatorname{Spec} \mathbb{k}, B)$. The duality functors of Lemma 2.2 in this case are simply $\mathrm{D}=\mathrm{RHom}_{A}(-, R)$ and $\mathrm{D}^{\mathrm{op}}=$ $\mathrm{RHom}_{A^{\mathrm{op}}}(-, R)$. In view of [YZ2 Proposition 1.3] we see that $R$ is a dualizing complex in the sense of Definition 2.3 if and only if it satisfies the conditions of Definition 2.7 
In Theorem 3.4 we show that the above is true for any pair of affine quasi-coherent ringed schemes.

We end this section with a couple of examples, that in fact digress from the main direction of our paper, yet are quite interesting on their own. The first is a contravariant Fourier-Mukai transform on elliptic curves.

Proposition 2.9. Let $X$ be an elliptic curve over $\mathbb{k}:=\mathbb{C}$ and let $\mathcal{R}$ be the Poincaré bundle on $X^{2}$. Then $\left(X^{2}, \mathcal{O}_{X^{2}}\right)$ is the product of the quasi-coherent ringed scheme $\left(X, \mathcal{O}_{X}\right)$ with itself, and $\mathcal{R} \in \mathrm{D}_{\mathrm{c}}^{\mathrm{b}}\left(\operatorname{Mod} \mathcal{O}_{X^{2}}\right)$ is a dualizing complex over $\mathcal{O}_{X}$ in the sense of Definition [2.5.

Proof. Conditions (i)-(ii) of Definition 2.3 are clearly verified in this case, since $X^{2}$ is smooth and proper. To check (iii) it suffices to prove that the adjunction morphisms $\phi_{1}: \mathcal{O}_{X} \rightarrow \mathrm{D}^{\mathrm{op}} \mathrm{D} \mathcal{O}_{X}$ and $\phi_{2}: \mathcal{O}_{X} \rightarrow \mathrm{DD}^{\mathrm{op}} \mathcal{O}_{X}$ are isomorphisms.

We begin by showing that $\phi_{1}$ and $\phi_{2}$ are nonzero. Going over the adjunctions in the proof of Lemma 2.2 we see that $\mathrm{DO}_{X} \cong \mathrm{Rp}_{2 *} \mathcal{R}$ and

$$
\operatorname{Hom}_{\mathrm{D}\left(\operatorname{Mod} \mathcal{O}_{X}\right)}\left(\mathcal{O}_{X}, \mathrm{D}^{\mathrm{op}} \mathrm{D} \mathcal{O}_{X}\right) \cong \operatorname{Hom}_{\mathrm{D}\left(\operatorname{Mod} \mathcal{O}_{X}\right)}\left(\mathrm{Rp}_{2 *} \mathcal{R}, \mathrm{Rp}_{2 *} \mathcal{R}\right) \text {. }
$$

Under the second isomorphism $\phi_{1}$ corresponds to the identity morphism of $\mathrm{Rp}_{2 *} \mathcal{R}$, which is nonzero. Similarly $\phi_{2}$ is shown to be nonzero.

Next for any $\mathcal{M} \in \mathrm{D}_{\mathrm{c}}^{\mathrm{b}}\left(\operatorname{Mod} \mathcal{O}_{X}\right)$ define

$$
\begin{aligned}
& F \mathcal{M}:=\mathrm{Rp}_{2 *}\left(\mathrm{p}_{1}^{*} \mathcal{M} \otimes_{\mathcal{O}_{X^{2}}}^{\mathrm{L}} \mathcal{R}\right), \\
& F^{\mathrm{op}} \mathcal{M}:=\mathrm{Rp}_{1 *}\left(\mathrm{p}_{2}^{*} \mathcal{M} \otimes_{\mathcal{O}_{X^{2}}}^{\mathrm{L}} \mathcal{R}\right), \\
& E \mathcal{M}:=\operatorname{RHom}_{\mathcal{O}_{X}}\left(\mathcal{M}, \mathcal{O}_{X}\right) \text {. }
\end{aligned}
$$

We then have

$$
\begin{aligned}
\mathrm{D} \mathcal{M} & =\mathrm{Rp}_{2 *} \mathrm{RHom}_{\mathcal{O}_{X^{2}}}\left(\mathrm{p}_{1}^{*} \mathcal{M}, \mathcal{R}\right) \\
& \cong \mathrm{Rp}_{2 *}\left(\left(\mathrm{p}_{1}^{*} \mathrm{RHom}_{\mathcal{O}_{X}}\left(\mathcal{M}, \mathcal{O}_{X}\right)\right) \otimes_{\mathcal{O}_{X^{2}}}^{\mathrm{L}} \mathcal{R}\right)=F E \mathcal{M} .
\end{aligned}
$$

Likewise we get $\mathrm{D}^{\mathrm{op}} \mathcal{M} \cong F^{\mathrm{op}} E \mathcal{M}$.

Let $O \in X$ be the zero for the group structure and $Z:=\{O\}_{\text {red }}$. According to $\left[\mathrm{Mu}\right.$ ] the Fourier-Mukai transform satisfies $F \mathcal{O}_{X} \cong F^{\mathrm{op}} \mathcal{O}_{X} \cong \mathcal{O}_{Z}[-1]$ and $F \mathcal{O}_{Z} \cong$ $F^{\mathrm{op}} \mathcal{O}_{Z} \cong \mathcal{O}_{X}$. Because $E \mathcal{O}_{X} \cong \mathcal{O}_{X}$ and $E \mathcal{O}_{Z} \cong \mathcal{O}_{Z}[-1]$ we obtain

$$
\mathrm{DD}^{\mathrm{op}} \mathcal{O}_{X} \cong \mathrm{D}^{\mathrm{op}} \mathrm{D} \mathcal{O}_{X} \cong \mathcal{O}_{X}
$$

Finally we use the facts that $\operatorname{Hom}_{\mathrm{D}\left(\operatorname{Mod} \mathcal{O}_{X}\right)}\left(\mathcal{O}_{X}, \mathcal{O}_{X}\right) \cong \mathbb{C}$ and that $\phi_{1}$ and $\phi_{2}$ are nonzero to deduce that $\phi_{1}$ and $\phi_{2}$ are isomorphisms.

The previous result can most likely be extended to higher dimensional abelian varieties. The next example is based on a result of Beilinson [Be].

Proposition 2.10. Consider projective space $X:=\mathbf{P}_{\mathbb{k}}^{n}$. Let $\mathcal{E}:=\bigoplus_{i=0}^{n} \mathcal{O}_{X}(i)$ and $B:=\operatorname{End}_{\mathcal{O}_{X}}(\mathcal{E})^{\mathrm{op}}$. Then $\mathcal{E} \in \operatorname{Mod} \mathcal{O}_{X} \otimes B^{\mathrm{op}}$ is a dualizing complex over $\left(\mathcal{O}_{X}, B\right)$.

Proof. One has

$$
\mathrm{D} \mathcal{M}=\mathrm{Rp}_{2 *} \mathrm{RH} \operatorname{Hom}_{\mathcal{O}_{X}}(\mathcal{M}, \mathcal{E}) \cong \operatorname{RHom}_{\mathcal{O}_{X}}(\mathcal{M}, \mathcal{E})
$$

for every $\mathcal{M} \in \mathrm{D}_{\mathrm{c}}^{\mathrm{b}}\left(\operatorname{Mod} \mathcal{O}_{X}\right)$. Since $X$ is smooth and proper over $\mathbb{k}$, this shows that D satisfies conditions (i) and (ii) of Definition 2.3.

On the other hand

$$
\mathrm{D}^{\mathrm{op}} N=\mathrm{Rp}_{1 *} \mathrm{RH}_{\mathcal{H}} m_{\mathrm{p}_{2}^{-1} B^{\mathrm{op}}}\left(\mathrm{p}_{2}^{-1} N, \mathcal{E}\right)
$$


for every $N \in \mathrm{D}_{\mathrm{f}}^{\mathrm{b}}\left(\operatorname{Mod} B^{\mathrm{op}}\right)$. It is known that $B$ is a finite $\mathbb{k}$-algebra of global dimension $n$. So any $N \in \operatorname{Mod}_{\mathrm{f}} B^{\mathrm{op}}$ has a resolution $P \rightarrow N$ with each $P^{i}$ a finite projective $B^{\mathrm{op}}$-module, and $P^{i}=0$ unless $-n \leq i \leq 0$. Because $\mathrm{D}^{\mathrm{op}} N \cong$ $\mathcal{H o m}_{\mathrm{p}_{2}^{-1} B^{\mathrm{op}}}\left(\mathrm{p}_{2}^{-1} P, \mathcal{E}\right)$ we see that the functor $\mathrm{D}^{\text {op }}$ satisfies conditions (i) and (ii).

It remains to verify that the adjunctions $\mathcal{M} \rightarrow \mathrm{D}^{\mathrm{op}} \mathrm{D} \mathcal{M}$ and $N \rightarrow \mathrm{DD}^{\mathrm{op}} N$ are isomorphisms.

Choose an injective resolution $\mathcal{E} \rightarrow \mathcal{J}$ over $\mathcal{O}_{X} \otimes B^{\text {op }}$. We then have $\mathrm{D} \mathcal{M} \cong$ $\operatorname{Hom}_{\mathcal{O}_{X}}(\mathcal{M}, \mathcal{J})$ and $\mathrm{D}^{\mathrm{op}} N \cong \mathcal{H}_{o m} \mathrm{p}_{2}^{-1} B^{\mathrm{op}}\left(\mathrm{p}_{2}^{-1} N, \mathcal{J}\right)$. Because $B$ and $\mathcal{E}$ generate the categories $\mathrm{D}_{\mathrm{f}}^{\mathrm{b}}\left(\operatorname{Mod} B^{\mathrm{op}}\right)$ and $\mathrm{D}_{\mathrm{c}}^{\mathrm{b}}\left(\operatorname{Mod} \mathcal{O}_{X}\right)$ respectively, it suffices to check that $\phi_{1}: \mathcal{E} \rightarrow \mathrm{D}^{\mathrm{op}} \mathrm{D} \mathcal{E}$ and $\phi_{2}: B \rightarrow \mathrm{DD}^{\mathrm{op}} B$ are isomorphisms. Now the fact that $\operatorname{Ext}_{\mathcal{O}_{X}}^{i}(\mathcal{E}, \mathcal{E})=0$ for all $i \neq 0$ implies that

$$
B \rightarrow \operatorname{Hom}_{\mathcal{O}_{X}}(\mathcal{J}, \mathcal{J}) \rightarrow \operatorname{Hom}_{\mathcal{O}_{X}}(\mathcal{E}, \mathcal{J})
$$

are quasi-isomorphisms. Therefore

$$
\mathcal{E} \stackrel{\phi_{1}}{\rightarrow} \mathcal{H o m}_{\mathrm{p}_{2}^{-1} B^{\mathrm{op}}}\left(\mathrm{p}_{2}^{-1} \operatorname{Hom}_{\mathcal{O}_{X}}(\mathcal{E}, \mathcal{J}), \mathcal{J}\right) \rightarrow \mathcal{J}
$$

and

$$
B \stackrel{\phi_{2}}{\longrightarrow} \operatorname{Hom}_{\mathcal{O}_{X}}\left(\mathcal{H o m} \mathrm{p}_{2}^{-1} B_{\mathrm{op}}\left(\mathrm{p}_{2}^{-1} B, \mathcal{J}\right), \mathcal{J}\right) \rightarrow \operatorname{Hom}_{\mathcal{O}_{X}}(\mathcal{J}, \mathcal{J})
$$

are quasi-isomorphisms.

The crucial fact in Proposition 2.10 is that $\mathcal{O}_{X}, \mathcal{O}_{X}(1), \ldots, \mathcal{O}_{X}(n)$ is an exceptional sequence in the sense of $[\mathrm{BO}$. This result can be extended to other smooth complete varieties that admit exceptional sequences.

\section{Rigid Dualizing Complexes}

We are mainly interested in dualizing complexes on ringed schemes that have a local behavior - as opposed to, say, the dualizing complexes occurring in Propositions 2.9 and 2.10]

Definition 3.1. Let $(X, \mathcal{A})$ and $(Y, \mathcal{B})$ be separated quasi-coherent ringed schemes over $\mathbb{k}$, and let $\mathcal{R} \in \mathrm{D}_{\mathrm{qc}}^{\mathrm{b}}\left(\operatorname{Mod} \mathcal{A} \otimes \mathcal{B}^{\mathrm{op}}\right)$ be a dualizing complex over $(\mathcal{A}, \mathcal{B})$. If the support of $\mathcal{R}$ (i.e. the union of the supports of the cohomology sheaves $\mathrm{H}^{i} \mathcal{R}$ ) is contained in the graph of an isomorphism of schemes $X \stackrel{\simeq}{\rightarrow} Y$ then we call $\mathcal{R}$ a local dualizing complex.

Lemma 3.2. Let $(X, \mathcal{A})$ be a left noetherian quasi-coherent ringed scheme, $U \subset X$ an open set and $\mathcal{M}$ a coherent $\left.\mathcal{A}\right|_{U}$-module. Then $\mathcal{M}$ extends to a coherent $\mathcal{A}$ module.

Proof. Let $g: U \rightarrow X$ be the inclusion. The sheaf $g_{*} \mathcal{M}$ is a quasi-coherent $\mathcal{O}_{X^{-}}$ module, hence it is a quasi-coherent $\mathcal{A}$-module. Also $g_{*} \mathcal{M}=\bigcup_{\alpha} \mathcal{L}_{\alpha}$ where $\left\{\mathcal{L}_{\alpha}\right\}$ is the set of its coherent $\mathcal{O}_{X}$-submodules (cf. [EGA I Corollary 6.9.9]). For any $\alpha$ the image

$$
\mathcal{N}_{\alpha}:=\operatorname{Im}\left(\mathcal{A} \otimes_{\mathcal{O}_{X}} \mathcal{L}_{\alpha} \rightarrow g_{*} \mathcal{M}\right)
$$

is a coherent $\mathcal{A}$-module. Now

$$
\mathcal{M}=\left.\left(g_{*} \mathcal{M}\right)\right|_{U}=\bigcup_{\alpha}\left(\left.\mathcal{N}_{\alpha}\right|_{U}\right),
$$

and because $\mathcal{M}$ is a noetherian object we get $\mathcal{M}=\left.\mathcal{N}_{\alpha}\right|_{U}$ for some $\alpha$. 
Proposition 3.3. Let $(X, \mathcal{A})$ and $(Y, \mathcal{B})$ be separated quasi-coherent ringed schemes over $\mathbb{k}$. Assume $\mathcal{A}$ and $\mathcal{B}^{\text {op }}$ are left noetherian and the product $\left(X \times Y, \mathcal{A} \otimes \mathcal{B}^{\mathrm{op}}\right)$ exists. Let $\mathcal{R} \in \mathrm{D}_{\mathrm{qc}}^{\mathrm{b}}\left(\operatorname{Mod} \mathcal{A} \otimes \mathcal{B}^{\mathrm{op}}\right)$ be a complex, and let $X=\bigcup U_{i}$ be any open covering of $X$. Then the following two conditions are equivalent.

(i) $\mathcal{R}$ is a local dualizing complex over $(\mathcal{A}, \mathcal{B})$.

(ii) $\mathcal{R}$ is supported on the graph of some isomorphism $f: X \stackrel{\simeq}{\rightarrow} Y$, and for every $i$, defining $V_{i}:=f\left(U_{i}\right)$, the restriction

$$
\left.\mathcal{R}\right|_{U_{i} \times V_{i}} \in \mathrm{D}_{\mathrm{qc}}^{\mathrm{b}}\left(\left.\operatorname{Mod}\left(\mathcal{A} \otimes \mathcal{B}^{\mathrm{op}}\right)\right|_{U_{i} \times V_{i}}\right)
$$

is a dualizing complex over $\left(\left.\mathcal{A}\right|_{U_{i}},\left.\mathcal{B}\right|_{V_{i}}\right)$.

Proof. Assume $\mathcal{R}$ has support in the graph of an isomorphism $f: X \rightarrow Y$. We shall write $\mathrm{p}_{(1, i)}: U_{i} \times V_{i} \rightarrow U_{i}$ and $\mathrm{p}_{(2, i)}: U_{i} \times V_{i} \rightarrow V_{i}$ for the projections on the open sets. Also we denote by $\mathrm{D}_{i}$ and $\mathrm{D}_{i}^{\text {op }}$ the duality functors determined by $\left.\mathcal{R}\right|_{U_{i} \times V_{i}}$. For any $\mathcal{M} \in \mathrm{D}^{\mathrm{b}}(\operatorname{Mod} \mathcal{A})$ we have

$$
\begin{aligned}
\left.(\mathrm{D} \mathcal{M})\right|_{V_{i}} & =\left.\left(\mathrm{Rp}_{2 *} \mathrm{RHom}_{\mathrm{p}_{1}^{-1} \mathcal{A}}\left(\mathrm{p}_{1}^{-1} \mathcal{M}, \mathcal{R}\right)\right)\right|_{V_{i}} \\
& \cong \operatorname{Rp}_{(2, i) *}\left(\left.\mathrm{RHom}_{\mathrm{p}_{1}^{-1} \mathcal{A}}\left(\mathrm{p}_{1}^{-1} \mathcal{M}, \mathcal{R}\right)\right|_{U_{i} \times V_{i}}\right) \\
& \cong \operatorname{Rp}_{(2, i) *}\left(\left.\operatorname{RHom}_{\mathrm{p}_{(1, i)}^{-1} \mathcal{A}}\left(\left.\mathrm{p}_{(1, i)}^{-1} \mathcal{M}\right|_{U_{i}}, \mathcal{R}\right)\right|_{U_{i} \times V_{i}}\right)=\mathrm{D}_{i}\left(\left.\mathcal{M}\right|_{U_{i}}\right) .
\end{aligned}
$$

Likewise $\left.\left(\mathrm{D}^{\mathrm{op}} \mathcal{N}\right)\right|_{U_{i}} \cong \mathrm{D}_{i}^{\mathrm{op}}\left(\left.\mathcal{N}\right|_{V_{i}}\right)$ for $\mathcal{N} \in \mathrm{D}^{\mathrm{b}}\left(\operatorname{Mod} \mathcal{B}^{\mathrm{op}}\right)$.

Any coherent $\mathcal{A}$-module restricts to a coherent $\left.\mathcal{A}\right|_{U_{i}}$-module, and the same for coherent $\mathcal{B}^{\mathrm{op}}$-modules. On the other hand by Lemma 3.2 any coherent $\left.\mathcal{A}\right|_{U_{i}}$-module extends to a coherent $\mathcal{A}$-module; and of course the same for coherent $\left.\mathcal{B}\right|_{V_{i}}$-modules. The upshot is that the three conditions of Definition 2.3 are satisfied for $\mathcal{R}$ if and only if they are satisfied for all the complexes $\left.\mathcal{R}\right|_{U_{i} \times V_{i}}$.

Suppose we are given $\mathbb{k}$-algebras $A$ and $B$, and we view them as quasi-coherent ringed schemes $(\operatorname{Spec} \mathbb{k}, A)$ and $(\operatorname{Spec} \mathbb{k}, B)$. As observed earlier in Example 2.8 for a complex $R \in \mathrm{D}^{\mathrm{b}}\left(\operatorname{Mod} A \otimes B^{\mathrm{op}}\right)$ Definition 2.3 becomes equivalent to the ringtheoretic Definition 2.7 As the next theorem shows the "same" is true for all affine quasi-coherent ringed schemes.

Theorem 3.4. Let $(X, \mathcal{A})$ and $(Y, \mathcal{B})$ be affine quasi-coherent ringed schemes over $\mathbb{k}$. Assume $\mathcal{A}$ and $\mathcal{B}^{\mathrm{op}}$ are left noetherian and the product $\left(X \times Y, \mathcal{A} \otimes \mathcal{B}^{\mathrm{op}}\right)$ exists. Let $\mathcal{R} \in \mathrm{D}_{\mathrm{qc}}^{\mathrm{b}}\left(\operatorname{Mod} \mathcal{A} \otimes \mathcal{B}^{\mathrm{op}}\right)$ be some complex. Define $A:=\Gamma(X, \mathcal{A})$ and $B:=\Gamma(Y, \mathcal{B})$. Then the following two conditions are equivalent.

(i) $\mathcal{R}$ is a dualizing complex over $(\mathcal{A}, \mathcal{B})$ in the sense of Definition 2.3 .

(ii) The complex

$$
R:=\mathrm{R} \Gamma(X \times Y, \mathcal{R}) \in \mathrm{D}\left(\operatorname{Mod} A \otimes B^{\mathrm{op}}\right)
$$

is a dualizing complex over $(A, B)$ in the sense of Definition 2.7

Proof. Let us write $\mathrm{D}_{\text {glob }}:=\operatorname{RHom}_{A}(-, R)$ and $\mathrm{D}_{\text {glob }}^{\text {op }}:=\operatorname{RHom}_{B^{\mathrm{op}}}(-, R)$. According to Corollary 1.18 the sheafification functors

$$
\begin{aligned}
& \mathcal{A} \otimes_{A}-: \mathrm{D}_{\mathrm{f}}^{\mathrm{b}}(\operatorname{Mod} A) \rightarrow \mathrm{D}_{\mathrm{c}}^{\mathrm{b}}(\operatorname{Mod} \mathcal{A}), \\
& -\otimes_{B} \mathcal{B}: \mathrm{D}_{\mathrm{f}}^{\mathrm{b}}\left(\operatorname{Mod} B^{\mathrm{op}}\right) \rightarrow \mathrm{D}_{\mathrm{c}}^{\mathrm{b}}\left(\operatorname{Mod} \mathcal{B}^{\mathrm{op}}\right)
\end{aligned}
$$


are equivalences, with inverses $\mathrm{R} \Gamma(X,-)$ and $\mathrm{R} \Gamma(Y,-)$ respectively. Thus it suffices to show that the diagram

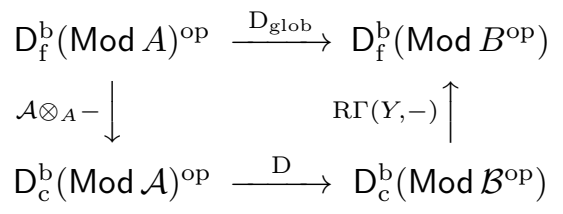

and the "opposite" diagram (the one involving $\mathrm{D}^{\mathrm{op}}$ and $\mathrm{D}_{\text {glob }}^{\mathrm{op}}$ ) are commutative. By symmetry it suffices to check only one of them, say the one displayed.

We can assume that $\mathcal{R}=\left(\mathcal{A} \otimes \mathcal{B}^{\mathrm{op}}\right) \otimes_{A \otimes B^{\mathrm{op}}} R$. Choose an injective resolution $R \rightarrow J$ in $\mathrm{C}^{+}\left(\operatorname{Mod} A \otimes B^{\mathrm{op}}\right)$, and let $\mathcal{J}:=\left(\mathcal{A} \otimes \mathcal{B}^{\mathrm{op}}\right) \otimes_{A \otimes B^{\text {op }}} J$. Then $\mathcal{R} \rightarrow \mathcal{J}$ is a quasi-isomorphism. Now $\mathcal{J}$ is a complex of injectives in QCoh $\mathcal{A} \otimes \mathcal{B}^{\text {op }}$, but it might not be a complex of injectives in Mod $\mathcal{A} \otimes \mathcal{B}^{\circ}$; cf. Remark 1.16 So we choose an injective resolution $\mathcal{J} \rightarrow \mathcal{K}$ in $\mathrm{C}^{+}\left(\operatorname{Mod} \mathcal{A} \otimes \mathcal{B}^{\text {op }}\right)$. Let $\mathcal{M}:=\mathcal{A} \otimes_{A} M \in \mathrm{D}_{\mathrm{c}}^{\mathrm{b}}(\operatorname{Mod} \mathcal{A})$. Then

$$
\mathrm{D} \mathcal{M}=\mathrm{p}_{2 *} \mathcal{H} o m_{\mathrm{p}_{1}^{-1} \mathcal{A}}\left(\mathrm{p}_{1}^{-1} \mathcal{M}, \mathcal{K}\right)
$$

Since the latter is a complex of flasque $\mathcal{B}^{\text {op }}$-modules on $Y$, we get

$$
\begin{aligned}
\mathrm{R} \Gamma(Y, \mathrm{D} \mathcal{M}) & =\Gamma\left(Y, \mathrm{p}_{2 *} \mathcal{H} o m_{\mathrm{p}_{1}^{-1} \mathcal{A}}\left(\mathrm{p}_{1}^{-1} \mathcal{M}, \mathcal{K}\right)\right) \\
& \cong \Gamma\left(X \times Y, \mathcal{H} m_{\mathrm{p}_{1}^{-1} \mathcal{A}}\left(\mathrm{p}_{1}^{-1} \mathcal{M}, \mathcal{K}\right)\right) \\
& \cong \operatorname{Hom}_{\mathrm{p}_{1}^{-1} \mathcal{A}}\left(\mathrm{p}_{1}^{-1} \mathcal{M}, \mathcal{K}\right) .
\end{aligned}
$$

Now choose a bounded above resolution $P \rightarrow M$ by finitely generated free $A$ modules, and let $\mathcal{P}:=\mathcal{A} \otimes_{A} P$. Then $\mathrm{p}_{1}^{-1} \mathcal{P} \rightarrow \mathrm{p}_{1}^{-1} \mathcal{M}$ is a quasi-isomorphism of $\mathrm{p}_{1}^{-1} \mathcal{A}$-modules, and so

$$
\operatorname{Hom}_{\mathrm{p}_{1}^{-1} \mathcal{A}}\left(\mathrm{p}_{1}^{-1} \mathcal{M}, \mathcal{K}\right) \rightarrow \operatorname{Hom}_{\mathrm{p}_{1}^{-1} \mathcal{A}}\left(\mathrm{p}_{1}^{-1} \mathcal{P}, \mathcal{K}\right)
$$

is a quasi-isomorphism of $B^{\mathrm{op}}$-modules. Each $\mathcal{J}^{p}$ and $\mathcal{K}^{p}$ is acyclic for the functor $\Gamma(X \times Y,-)$, since $\mathcal{J}^{p}$ is quasi-coherent and $\mathcal{K}^{p}$ is injective. Therefore $\Gamma(X \times Y, \mathcal{J}) \rightarrow$ $\Gamma(X \times Y, \mathcal{K})$ is a quasi-isomorphism. Now $\mathrm{p}_{1}^{-1} \mathcal{P}$ is a bounded above complex of finitely generated free $\mathrm{p}_{1}^{-1} \mathcal{A}$-modules, and thus

$$
\operatorname{Hom}_{\mathrm{p}_{1}^{-1} \mathcal{A}}\left(\mathrm{p}_{1}^{-1} \mathcal{P}, \mathcal{J}\right) \rightarrow \operatorname{Hom}_{\mathrm{p}_{1}^{-1} \mathcal{A}}\left(\mathrm{p}_{1}^{-1} \mathcal{P}, \mathcal{K}\right)
$$

is a quasi-isomorphism of $B^{\text {op }}$-modules. But

$$
\operatorname{Hom}_{\mathrm{p}_{1}^{-1} \mathcal{A}}\left(\mathrm{p}_{1}^{-1} \mathcal{P}, \mathcal{J}\right) \cong \operatorname{Hom}_{A}(P, J)=\operatorname{RHom}_{A}(M, R)=\mathrm{D}_{\text {glob }} M .
$$

Next we wish to recall the definition of rigid dualizing complex over a $\mathbb{k}$-algebra, which is due to Van den Bergh VdB1.

Definition 3.5. Let $R$ be a dualizing complex over $A$. If there is an isomorphism

$$
\rho: R \rightarrow \operatorname{RHom}_{A^{\mathrm{e}}}(A, R \otimes R)
$$

in $\mathrm{D}\left(\operatorname{Mod} A^{\mathrm{e}}\right)$ then we call $(R, \rho)$, or just $R$, a rigid dualizing complex. The isomorphism $\rho$ is called a rigidifying isomorphism. 
For a detailed explanation of this definition see [YZ2, Section 3]. According to VdB1, Proposition 8.2] and YZ2, Corollary 3.4] a rigid dualizing complex $(R, \rho)$ is unique up to a unique isomorphism in $\mathrm{D}\left(\operatorname{Mod} A^{\mathrm{e}}\right)$. It is important to note that rigidity is a relative notion (relative to the base field $\mathbb{k}$ ).

A ring homomorphism $A \rightarrow A^{\prime}$ is called a localization (in the sense of Silver [Si]) if $A^{\prime}$ is a flat $A$-module on both sides and $A^{\prime} \otimes_{A} A^{\prime} \cong A^{\prime}$.

Definition 3.6. Let $A \rightarrow A^{\prime}$ be a localization homomorphism between two noetherian $\mathbb{k}$-algebras. Suppose the rigid dualizing complexes $(R, \rho)$ and $\left(R^{\prime}, \rho^{\prime}\right)$ of $A$ and $A^{\prime}$ respectively exist. A rigid localization morphism is a morphism

$$
\mathrm{q}_{A^{\prime} / A}: R \rightarrow R^{\prime}
$$

in $\mathrm{D}\left(\operatorname{Mod} A^{\mathrm{e}}\right)$ satisfying the conditions below.

(i) The morphisms $A^{\prime} \otimes_{A} R \rightarrow R^{\prime}$ and $R \otimes_{A} A^{\prime} \rightarrow R^{\prime}$ in $\mathrm{D}\left(\operatorname{Mod} A^{\mathrm{e}}\right)$ induced by $\mathrm{q}_{A^{\prime} / A}$ are isomorphisms.

(ii) The diagram

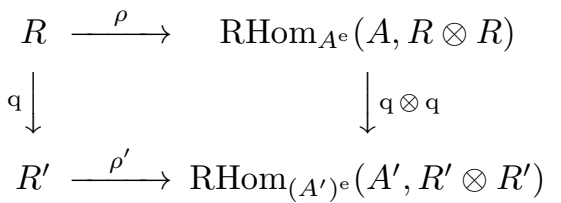

in $\mathrm{D}\left(\operatorname{Mod} A^{\mathrm{e}}\right)$, where $\mathrm{q}:=\mathrm{q}_{A^{\prime} / A}$, is commutative.

We shall sometimes express this by saying that $\mathrm{q}_{A^{\prime} / A}:(R, \rho) \rightarrow\left(R^{\prime}, \rho^{\prime}\right)$ is a rigid localization morphism.

According to [YZ4, Theorem 6.2] a rigid localization morphism $\mathrm{q}_{A^{\prime} / A}:(R, \rho) \rightarrow$ $\left(R^{\prime}, \rho^{\prime}\right)$ is unique (if it exists).

Let us denote by Aff $X$ the set of affine open subsets of $X$. Suppose $\mathcal{R}$ is a local dualizing complex over $\mathcal{A}$ supported on the diagonal $\Delta(X) \subset X^{2}$. By Proposition 3.3 and Theorem 3.4 for every $U \in \operatorname{Aff} X$ the complex $\operatorname{R} \Gamma\left(U^{2}, \mathcal{R}\right)$ is a dualizing complex over $A:=\Gamma(U, \mathcal{A})$. If $V \subset U$ is another affine open set and $A^{\prime}:=\Gamma(V, \mathcal{A})$ then both $A \rightarrow A^{\prime}$ and $A^{\mathrm{e}} \rightarrow A^{\prime \mathrm{e}}$ are localizations. Moreover the restriction $\mathrm{D}_{\mathrm{c}}^{\mathrm{b}}\left(\left.\operatorname{Mod} \mathcal{A}^{\mathrm{e}}\right|_{U^{2}}\right) \rightarrow \mathrm{D}_{\mathrm{c}}^{\mathrm{b}}\left(\left.\operatorname{Mod} \mathcal{A}^{\mathrm{e}}\right|_{V^{2}}\right)$ induces a morphism $\mathrm{q}_{A^{\prime} / A}: R \rightarrow R^{\prime}$ in $\mathrm{D}\left(\operatorname{Mod} A^{\mathrm{e}}\right)$; cf. Lemma 1.19

Definition 3.7. Let $(X, \mathcal{A})$ be a separated noetherian quasi-coherent ringed scheme over $\mathbb{k}$. Assume the product $\left(X^{2}, \mathcal{A}^{\mathrm{e}}\right)$ exists, and is also noetherian. A rigid dualizing complex over $\mathcal{A}$ is a pair $(\mathcal{R}, \boldsymbol{\rho})$, where:

(1) $\mathcal{R} \in \mathrm{D}_{\mathrm{c}}^{\mathrm{b}}\left(\operatorname{Mod} \mathcal{A}^{\mathrm{e}}\right)$ is a local dualizing complex over $\mathcal{A}$ supported on the diagonal $\Delta(X) \subset X^{2}$.

(2) $\boldsymbol{\rho}=\left\{\rho_{U}\right\}_{U \in \text { Aff } X}$ is a collection of rigidifying isomorphisms, namely for each $U \in$ Aff $X$, letting $A:=\Gamma(U, \mathcal{A})$ and $R:=\mathrm{R} \Gamma\left(U^{2}, \mathcal{R}\right)$, the pair $\left(R, \rho_{U}\right)$ is a rigid dualizing complex over $A$.

The following compatibility condition is required of the data $(\mathcal{R}, \rho)$ :

(*) Given $V \subset U$ in Aff $X$, write $A^{\prime}:=\Gamma(V, \mathcal{A})$ and $R^{\prime}:=\mathrm{R} \Gamma\left(V^{2}, \mathcal{R}\right)$. Let $\mathrm{q}_{A^{\prime} / A}: R \rightarrow R^{\prime}$ be the morphism in $\mathrm{D}\left(\operatorname{Mod} A^{\mathrm{e}}\right)$ coming from restriction. Then

$$
\mathrm{q}_{A^{\prime} / A}:\left(R, \rho_{U}\right) \rightarrow\left(R^{\prime}, \rho_{V}\right)
$$

is a rigid localization morphism. 
Example 3.8. Let $X$ be a separated finite type $\mathbb{k}$-scheme, smooth of dimension $n$. There is a canonical isomorphism

$$
\rho: \Delta_{*} \Omega_{X / \mathbb{k}}^{n} \stackrel{\simeq}{\rightarrow} \mathcal{E} x t_{\mathcal{O}_{X^{2}}}^{n}\left(\Delta_{*} \mathcal{O}_{X}, \Omega_{X^{2} / \mathbb{k}}^{2 n}\right)
$$

and $\mathcal{E}_{x} t_{\mathcal{O}_{X^{2}}}^{i}\left(\Delta_{*} \mathcal{O}_{X}, \Omega_{X^{2} / \mathbb{k}}^{2 n}\right)=0$ for $i \neq n$ (see $\underline{\mathrm{RD}}$ Proposition III.7.2]). Therefore on any affine open set $U=\operatorname{Spec} A \subset X$ we get an isomorphism

$$
\rho_{U}: \Omega_{A / \mathbb{k}}^{n}[n] \stackrel{\simeq}{\rightarrow} \operatorname{RHom}_{A^{\mathrm{e}}}\left(A, \Omega_{A / \mathbb{k}}^{n}[n] \otimes \Omega_{A / \mathbb{k}}^{n}[n]\right)
$$

in $\mathrm{D}\left(\operatorname{Mod} A^{\mathrm{e}}\right)$, and the collection $\boldsymbol{\rho}:=\left\{\rho_{U}\right\}$ is compatible with localization. We see that $\left(\Delta_{*} \Omega_{A / \mathbb{k}}^{n}[n], \boldsymbol{\rho}\right)$ is a rigid dualizing complex over $X$ in the sense of Definition 3.7

Definition 3.9. A morphism $f:(Y, \mathcal{B}) \rightarrow(X, \mathcal{A})$ between noetherian quasicoherent ringed schemes is called finite if $f: Y \rightarrow X$ is finite and $f_{*} \mathcal{B}$ is a coherent $\mathcal{A}$-module on both sides.

Given a morphism $f:(Y, \mathcal{B}) \rightarrow(X, \mathcal{A})$ of ringed schemes then $f^{\mathrm{op}}:\left(Y, \mathcal{B}^{\mathrm{op}}\right) \rightarrow$ $\left(X, \mathcal{A}^{\mathrm{op}}\right)$ is also a morphism. According to Proposition 1.6 if the products exist then there is a morphism

$$
f^{\mathrm{e}}:=f \times f^{\mathrm{op}}:\left(Y^{2}, \mathcal{B}^{\mathrm{e}}\right) \rightarrow\left(X^{2}, \mathcal{A}^{\mathrm{e}}\right) .
$$

Definition 3.10. Let $A \rightarrow B$ be a finite homomorphism of $\mathbb{k}$-algebras. Assume the rigid dualizing complexes $\left(R_{A}, \rho_{A}\right)$ and $\left(R_{B}, \rho_{B}\right)$ exist. Let $\operatorname{Tr}_{B / A}: R_{B} \rightarrow R_{A}$ be a morphism in $\mathrm{D}\left(\operatorname{Mod} A^{\mathrm{e}}\right)$. We say $\operatorname{Tr}_{B / A}$ is a rigid trace if it satisfies the following two conditions:

(i) $\operatorname{Tr}_{B / A}$ induces isomorphisms

$$
R_{B} \cong \operatorname{RHom}_{A}\left(B, R_{A}\right) \cong \operatorname{RHom}_{A^{\text {op }}}\left(B, R_{A}\right)
$$

in $\mathrm{D}\left(\operatorname{Mod} A^{\mathrm{e}}\right)$.

(ii) The diagram

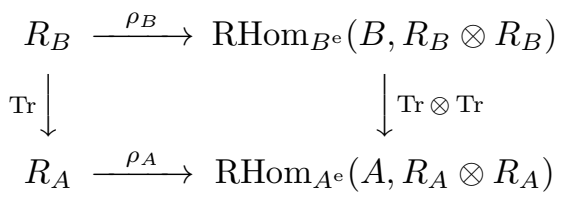

in $\mathrm{D}\left(\operatorname{Mod} A^{\mathrm{e}}\right)$, where $\operatorname{Tr}:=\operatorname{Tr}_{B / A}$, is commutative.

Often we shall say that $\operatorname{Tr}_{B / A}:\left(R_{B}, \rho_{B}\right) \rightarrow\left(R_{A}, \rho_{A}\right)$ is a rigid trace morphism.

By [YZ2 Theorem 3.2], a rigid trace $\operatorname{Tr}_{B / A}$ is unique (if it exists).

Definition 3.11. Let $f:(Y, \mathcal{B}) \rightarrow(X, \mathcal{A})$ be a finite morphism between noetherian separated quasi-coherent ringed $\mathbb{k}$-schemes. Assume both $(X, \mathcal{A})$ and $(Y, \mathcal{B})$ have rigid dualizing complexes $\left(\mathcal{R}_{\mathcal{A}}, \boldsymbol{\rho}_{\mathcal{A}}\right)$ and $\left(\mathcal{R}_{\mathcal{B}}, \boldsymbol{\rho}_{\mathcal{B}}\right)$ respectively. A rigid trace is a morphism

$$
\operatorname{Tr}_{f}: \mathrm{R} f_{*}^{\mathrm{e}} \mathcal{R}_{\mathcal{B}} \rightarrow \mathcal{R}_{\mathcal{A}}
$$

in $\mathrm{D}\left(\operatorname{Mod} \mathcal{A}^{\mathrm{e}}\right)$ satisfying the following condition.

(†) Let $U \subset X$ be any affine open set, $V:=f^{-1}(U), A:=\Gamma(U, \mathcal{A})$ and $B:=$ $\Gamma(V, \mathcal{B})$. Let $R_{A}:=\mathrm{R} \Gamma\left(U^{2}, \mathcal{R}_{\mathcal{A}}\right)$ and $R_{B}:=\mathrm{R} \Gamma\left(V^{2}, \mathcal{R}_{\mathcal{B}}\right)$ be the rigid dualizing complexes, with their respective rigidifying isomorphisms $\rho_{U}$ and $\rho_{V}$. Then

$$
\mathrm{R} \Gamma\left(U^{2}, \operatorname{Tr}_{f}\right):\left(R_{B}, \rho_{V}\right) \rightarrow\left(R_{A}, \rho_{U}\right)
$$


is a rigid trace morphism.

Example 3.12. Suppose $X$ and $Y$ are separated $\mathbb{k}$-schemes, smooth of dimensions $m$ and $n$ respectively. Let $f: X \rightarrow Y$ be a finite morphism. According to [RD Theorem III.10.5] there is a trace morphism $\operatorname{Tr}_{f}: f_{*} \Omega_{X / \mathrm{k}}^{m}[m] \rightarrow \Omega_{Y / \mathrm{k}}^{n}[n]$ in $\mathrm{D}\left(\operatorname{Mod} \mathcal{O}_{Y}\right)$. We know that $\Delta_{*} \Omega_{X / \mathrm{k}}^{m}[m]$ and $\Delta_{*} \Omega_{Y / \mathrm{k}}^{n}[n]$ are the rigid dualizing complexes of these schemes (see Example 3.8). Condition TRA2 of RD, Theorem III.10.2] implies that $\Delta_{*}\left(\operatorname{Tr}_{f}\right)$ it is a rigid trace in the sense of Definition 3.11

Remark 3.13. For $\mathbb{k}$-algebras one has the notion of Auslander dualizing complex, see YZ2. In YZ4 it was shown that the Auslander condition is closely related to the rigid perverse t-structure. It should be interesting to extend the Auslander condition to the geometric context, i.e. to dualizing complexes over quasi-coherent ringed schemes.

\section{Perverse Sheaves on Ringed Spaces}

This section deals with gluing t-structures, in a rather general context. Let us begin by recalling the following basic definition due to Beilinson, Bernstein and Deligne [BBD. We shall follow the exposition in [KS, Chapter X].

Definition 4.1. Suppose $D$ is a triangulated category and $D^{\leq 0}, D^{\geq 0}$ are two full subcategories. Let $\mathrm{D}^{\leq n}:=\mathrm{D}^{\leq 0}[-n]$ and $\mathrm{D}^{\geq n}:=\mathrm{D}^{\geq 0}[-n]$. We say $\left(\mathrm{D}^{\leq 0}, \mathrm{D}^{\geq 0}\right)$ is a $t$-structure on $\mathrm{D}$ if:

(i) $\mathrm{D}^{\leq-1} \subset \mathrm{D}^{\leq 0}$ and $\mathrm{D}^{\geq 1} \subset \mathrm{D}^{\geq 0}$.

(ii) $\operatorname{Hom}_{\mathrm{D}}(M, N)=0$ for $M \in \mathrm{D}^{\leq 0}$ and $N \in \mathrm{D}^{\geq 1}$.

(iii) For any $M \in \mathrm{D}$ there is a distinguished triangle

$$
M^{\prime} \rightarrow M \rightarrow M^{\prime \prime} \rightarrow M^{\prime}[1]
$$

in $\mathrm{D}$ with $M^{\prime} \in \mathrm{D}^{\leq 0}$ and $M^{\prime \prime} \in \mathrm{D}^{\geq 1}$.

When these conditions are satisfied we define the heart of $D$ to be the full subcategory $\mathrm{D}^{0}:=\mathrm{D}^{\leq 0} \cap \mathrm{D}^{\geq 0}$.

It is known that the heart $\mathrm{D}^{0}$ is an abelian category, in which short exact sequences are distinguished triangles in $\mathrm{D}$ with vertices in $\mathrm{D}^{0}$.

Let $(X, \mathcal{A})$ be a ringed space, i.e. a topological space $X$ endowed with a sheaf of (not necessarily commutative) rings $\mathcal{A}$. We denote by $\operatorname{Mod} \mathcal{A}$ the category of sheaves of left $\mathcal{A}$-modules, and by $\mathrm{D}(\operatorname{Mod} \mathcal{A})$ the derived category.

The triangulated category $\mathrm{D}(\operatorname{Mod} \mathcal{A})$ has the standard t-structure, in which

$$
\begin{aligned}
& \mathrm{D}(\operatorname{Mod} \mathcal{A})^{\leq 0}:=\left\{\mathcal{M} \in \mathrm{D}(\operatorname{Mod} \mathcal{A}) \mid \mathrm{H}^{i} \mathcal{M}=0 \text { for all } i>0\right\}, \\
& \mathrm{D}(\operatorname{Mod} \mathcal{A})^{\geq 0}:=\left\{\mathcal{M} \in \mathrm{D}(\operatorname{Mod} \mathcal{A}) \mid \mathrm{H}^{i} \mathcal{M}=0 \text { for all } i<0\right\} .
\end{aligned}
$$

The heart $\mathrm{D}(\operatorname{Mod} \mathcal{A})^{0}$ is equivalent to $\operatorname{Mod} \mathcal{A}$. Other t-structures on $\mathrm{D}(\operatorname{Mod} \mathcal{A})$, or on some triangulated full subcategory $\mathrm{D} \subset \mathrm{D}(\operatorname{Mod} \mathcal{A})$, will be called perverse $t$-structures, and the notation ${ }^{p} \mathrm{D}^{\star}$ shall be used.

A stack on $X$ is a "sheaf of categories." The general definition (cf. [LMB]) is quite forbidding; but we shall only need the following special instance (cf. KS Section $\mathrm{X} .10])$. Given two open sets $V \subset U$ in $X$, the restriction functor $\mathrm{D}\left(\left.\operatorname{Mod} \mathcal{A}\right|_{U}\right) \rightarrow$ $\mathrm{D}\left(\left.\operatorname{Mod} \mathcal{A}\right|_{V}\right)$ is denoted by $\left.\mathcal{M} \mapsto \mathcal{M}\right|_{V}$. 
Definition 4.2. Let $(X, \mathcal{A})$ be a ringed space. Suppose that for every open set $U \subset X$ we are given a full subcategory $\mathrm{C}(U) \subset \mathrm{D}\left(\left.\operatorname{Mod} \mathcal{A}\right|_{U}\right)$. The collection of categories $\mathrm{C}=\{\mathrm{C}(U)\}$ is called a stack of subcategories of $\mathrm{D}(\operatorname{Mod} \mathcal{A})$ if the following axioms hold.

(a) Let $V \subset U$ be open sets in $X$ and $\mathcal{M} \in \mathrm{C}(U)$. Then $\left.\mathcal{M}\right|_{V} \in \mathrm{C}(V)$.

(b) Descent for objects: given an open covering $U=\bigcup V_{i}$, objects $\mathcal{M}_{i} \in$ $\mathrm{C}\left(V_{i}\right)$ and isomorphisms $\phi_{i, j}:\left.\left.\mathcal{M}_{i}\right|_{V_{i} \cap V_{j}} \stackrel{\simeq}{\rightarrow} \mathcal{M}_{j}\right|_{V_{i} \cap V_{j}}$ satisfying the cocycle condition $\phi_{i, k}=\phi_{j, k} \circ \phi_{i, j}$ on triple intersections, there exists an object $\mathcal{M} \in \mathrm{C}(U)$ and isomorphisms $\phi_{i}:\left.\mathcal{M}\right|_{V_{i}} \stackrel{\simeq}{\rightarrow} \mathcal{M}_{i}$ such that $\phi_{i, j} \circ \phi_{i}=\phi_{j}$.

(c) Descent for morphisms: given two objects $\mathcal{M}, \mathcal{N} \in \mathrm{C}(U)$, an open covering $U=\bigcup V_{i}$ and morphisms $\psi_{i}:\left.\left.\mathcal{M}\right|_{V_{i}} \rightarrow \mathcal{N}\right|_{V_{i}}$ such that $\left.\psi_{i}\right|_{V_{i} \cap V_{j}}=\left.\psi_{j}\right|_{V_{i} \cap V_{j}}$, there is a unique morphism $\psi: \mathcal{M} \rightarrow \mathcal{N}$ such that $\left.\psi\right|_{V_{i}}=\psi_{i}$.

Unlike [LMB], our stacks do not consist of groupoids. On the contrary, we will work with stacks of abelian categories. Here is an example.

Example 4.3. Take $\mathrm{C}(U):=\mathrm{D}\left(\left.\operatorname{Mod} \mathcal{A}\right|_{U}\right)^{0}$, the heart for the standard t-structure. Since $\mathrm{C}(U)$ is canonically equivalent to $\left.\operatorname{Mod} \mathcal{A}\right|_{U}$ it follows that $\mathrm{C}=\{\mathrm{C}(U)\}$ is a stack of subcategories of $\mathrm{D}(\operatorname{Mod} \mathcal{A})$.

By abuse of notation we shall denote by $\operatorname{Mod} \mathcal{A}$ the stack $U \mapsto \mathrm{D}\left(\left.\operatorname{Mod} \mathcal{A}\right|_{U}\right)^{0}$.

Definition 4.4. Suppose $C$ is a stack of subcategories of $D(\operatorname{Mod} \mathcal{A})$ such that for any open set $U$ the subcategory $\mathrm{C}(U)$ is a thick abelian subcategory of $\mathrm{D}\left(\left.\operatorname{Mod} \mathcal{A}\right|_{U}\right)^{0}$. Then we call $\mathrm{C}$ a thick abelian substack of $\operatorname{Mod} \mathcal{A}$.

Example 4.5. Consider a noetherian scheme $\left(X, \mathcal{O}_{X}\right)$. Define $\mathrm{C}(U):=\operatorname{Coh} \mathcal{O}_{U}$, the category of coherent sheaves on $U$. This is a thick abelian substack of Mod $\mathcal{O}_{X}$, which we denote (by abuse of notation) $\operatorname{Coh} \mathcal{O}_{X}$.

From now until the end of this section we fix a ringed space $(X, \mathcal{A})$, a thick abelian substack $C \subset \operatorname{Mod} \mathcal{A}$ and a basis $\mathfrak{B}$ of the topology of $X$. For any open set $U \subset X$ the full subcategory $\mathrm{D}_{\mathrm{c}}^{\mathrm{b}}\left(\left.\operatorname{Mod} \mathcal{A}\right|_{U}\right)$, whose objects are the bounded complexes $\mathcal{M}$ such that $\mathrm{H}^{i} \mathcal{M} \in \mathrm{C}(U)$ for all $i$, is a triangulated subcategory of $\mathrm{D}^{\mathrm{b}}\left(\left.\operatorname{Mod} \mathcal{A}\right|_{U}\right)$.

Definition 4.6. Suppose that for every open set $U \in \mathfrak{B}$ we are given a t-structure

$$
\mathfrak{T}_{U}=\left({ }^{p} \mathrm{D}_{\mathrm{c}}^{\mathrm{b}}\left(\left.\operatorname{Mod} \mathcal{A}\right|_{U}\right) \leq 0,{ }^{p} \mathrm{D}_{\mathrm{c}}^{\mathrm{b}}\left(\left.\operatorname{Mod} \mathcal{A}\right|_{U}\right)^{\geq 0}\right)
$$

on $\mathrm{D}_{\mathrm{c}}^{\mathrm{b}}\left(\left.\operatorname{Mod} \mathcal{A}\right|_{U}\right)$. Furthermore suppose this collection of t-structures $\left\{\mathfrak{T}_{U}\right\}_{U \in \mathfrak{B}}$ satisfies the following condition.

$(\diamond)$ Let $U \in \mathfrak{B}$, let $U=\bigcup U_{i}$ be any covering with $U_{i} \in \mathfrak{B}$, and let " $\star$ " denote either " $\leq 0$ " or " $\geq 0$ ". Then for any $\mathcal{M} \in \mathrm{D}_{\mathrm{c}}^{\mathrm{b}}\left(\left.\operatorname{Mod} \mathcal{A}\right|_{U}\right)$ the following are equivalent:

(i) $\mathcal{M} \in{ }^{p} \mathrm{D}_{\mathrm{c}}^{\mathrm{b}}\left(\left.\operatorname{Mod} \mathcal{A}\right|_{U}\right)^{\star}$

(ii) $\left.\mathcal{M}\right|_{U_{i}} \in{ }^{p} D_{\mathrm{c}}^{\mathrm{b}}\left(\left.\operatorname{Mod} \mathcal{A}\right|_{U_{i}}\right)^{\star}$ for all $i$.

Then we call $\left\{\mathfrak{T}_{U}\right\}_{U \in \mathfrak{B}}$ a local collection of $t$-structures on $(X, \mathcal{A})$.

Definition 4.7. Let $\left\{\mathfrak{T}_{U}\right\}_{U \in \mathfrak{B}}$ be a local collection of t-structures on $(X, \mathcal{A})$. Define full subcategories

$$
\begin{aligned}
& { }^{p} \mathrm{D}_{\mathrm{c}}^{\mathrm{b}}(\operatorname{Mod} \mathcal{A})^{\star}:= \\
& \quad\left\{\mathcal{M} \in \mathrm{D}_{\mathrm{c}}^{\mathrm{b}}(\operatorname{Mod} \mathcal{A}) \quad|\mathcal{M}|_{U} \in{ }^{p} \mathrm{D}_{\mathrm{c}}^{\mathrm{b}}\left(\left.\operatorname{Mod} \mathcal{A}\right|_{U}\right)^{\star} \text { for every } U \in \mathfrak{B}\right\},
\end{aligned}
$$


where " $\star$ " is either " $\leq$ " or " $\geq "$.

The next lemmas are modifications of material in [KS Section 10.2]. We assume a local collection of t-structures $\left\{\mathfrak{T}_{U}\right\}_{U \in \mathfrak{B}}$ is given.

Lemma 4.8. Let $\mathcal{M} \in{ }^{p} \mathrm{D}_{\mathrm{c}}^{\mathrm{b}}(\operatorname{Mod} \mathcal{A}) \leq 0$ and $\mathcal{N} \in{ }^{p} \mathrm{D}_{\mathrm{c}}^{\mathrm{b}}(\operatorname{Mod} \mathcal{A})^{\geq 0}$.

(1) The sheaf $\mathrm{H}^{i} \mathrm{RHom} \operatorname{H}_{\mathcal{A}}(\mathcal{M}, \mathcal{N})$ vanishes for all $i \leq-1$.

(2) The assignment $U \mapsto \operatorname{Hom}_{\mathrm{D}\left(\left.\operatorname{Mod} \mathcal{A}\right|_{U}\right)}\left(\left.\mathcal{M}\right|_{U},\left.\mathcal{N}\right|_{U}\right)$, for open sets $U \subset X$, is a sheaf on $X$.

(3) $\operatorname{Hom}_{\mathrm{D}(\operatorname{Mod} \mathcal{A})}(\mathcal{M}, \mathcal{N}[i])=0$ for all $i \leq-1$.

Proof. (1) We note that

$$
\mathrm{H}^{i} \mathrm{RHom}_{\mathcal{A}}(\mathcal{M}, \mathcal{N}) \cong \mathrm{H}^{0} \mathrm{RHom}_{\mathcal{A}}(\mathcal{M}, \mathcal{N}[i]),
$$

and $\mathcal{N}[i] \in{ }^{p} \mathrm{D}_{\mathrm{c}}^{\mathrm{b}}(\operatorname{Mod} \mathcal{A})^{\geq 1}$, because $i \leq-1$.

Now $\mathrm{H}^{0} \mathrm{RHom} \operatorname{Hom}_{\mathcal{A}}(\mathcal{M}, \mathcal{N}[i])$ is isomorphic to the sheaf associated to the presheaf

$$
U \mapsto \mathrm{H}^{0} \operatorname{RHom}_{\left.\mathcal{A}\right|_{U}}\left(\left.\mathcal{M}\right|_{U},\left.\mathcal{N}[i]\right|_{U}\right) \cong \operatorname{Hom}_{\mathrm{D}\left(\left.\operatorname{Mod} \mathcal{A}\right|_{U}\right)}\left(\left.\mathcal{M}\right|_{U},\left.\mathcal{N}[i]\right|_{U}\right) .
$$

Thus it suffices to prove that

$$
\operatorname{Hom}_{\mathrm{D}\left(\left.\operatorname{Mod} \mathcal{A}\right|_{U}\right)}\left(\left.\mathcal{M}\right|_{U},\left.\mathcal{N}[i]\right|_{U}\right)=0
$$

for all open sets $U \in \mathfrak{B}$. Since by Definition 4.7 we have $\left.\mathcal{M}\right|_{U} \in{ }^{p} \mathrm{D}_{\mathrm{c}}^{\mathrm{b}}\left(\left.\operatorname{Mod} \mathcal{A}\right|_{U} \leq 0\right.$ and $\left.\mathcal{N}[i]\right|_{U} \in{ }^{p} \mathrm{D}_{\mathrm{c}}^{\mathrm{b}}\left(\left.\operatorname{Mod} \mathcal{A}\right|_{U}\right)^{\geq 1}$, the assertion follows from condition (ii) in Definition 4.1

(2) Let us write $\mathcal{L}:=\operatorname{RHom}_{\mathcal{A}}(\mathcal{M}, \mathcal{N}) \in \mathrm{D}\left(\operatorname{Mod} \mathbb{Z}_{X}\right)$. By part (1) we know that $\mathrm{H}^{i} \mathcal{L}=0$ for all $i<0$. So after truncation we can assume $\mathcal{L}^{i}=0$ for all $i<0$. Hence $\mathrm{H}^{0} \mathrm{R} \Gamma(U, \mathcal{L}) \cong \Gamma\left(U, \mathrm{H}^{0} \mathcal{L}\right)$. But

$$
\mathrm{H}^{0} \mathrm{R} \Gamma(U, \mathcal{L}) \cong \operatorname{Hom}_{\mathrm{D}\left(\left.\operatorname{Mod} \mathcal{A}\right|_{U}\right)}\left(\left.\mathcal{M}\right|_{U},\left.\mathcal{N}\right|_{U}\right)
$$

We see that the presheaf $U \mapsto \operatorname{Hom}_{\mathrm{D}\left(\left.\operatorname{Mod} \mathcal{A}\right|_{U}\right)}\left(\left.\mathcal{M}\right|_{U},\left.\mathcal{N}\right|_{U}\right)$ is actually a sheaf, namely the sheaf $\mathrm{H}^{0} \mathcal{L}$.

(3) Here $\mathcal{N}[i] \in{ }^{p} \mathrm{D}_{\mathrm{c}}^{\mathrm{b}}(\operatorname{Mod} \mathcal{A})^{\geq 1} \subset{ }^{p} \mathrm{D}_{\mathrm{c}}^{\mathrm{b}}(\operatorname{Mod} \mathcal{A})^{\geq 0}$, so equation (4.9) holds for all open sets in $\mathfrak{B}$. Applying part (2) of the lemma to $\mathcal{M}$ and $\mathcal{N}[i]$ we get $\operatorname{Hom}_{\mathrm{D}(\operatorname{Mod} \mathcal{A})}(\mathcal{M}, \mathcal{N}[i])=0$.

Lemma 4.10. Suppose the two rows in the diagram below are distinguished triangles with $\mathcal{M}_{1}^{\prime} \in{ }^{p} \mathrm{D}_{\mathrm{c}}^{\mathrm{b}}(\operatorname{Mod} \mathcal{A})^{\leq 0}$ and $\mathcal{M}_{2}^{\prime \prime} \in{ }^{p} \mathrm{D}_{\mathrm{c}}^{\mathrm{b}}(\operatorname{Mod} \mathcal{A})^{\geq 1}$. Given any morphism $g: \mathcal{M}_{1} \rightarrow \mathcal{M}_{2}$ there exist unique morphisms $f: \mathcal{M}_{1}^{\prime} \rightarrow \mathcal{M}_{2}^{\prime}$ and $h: \mathcal{M}_{1}^{\prime \prime} \rightarrow \mathcal{M}_{2}^{\prime \prime}$ making the diagram commutative. If $g$ is an isomorphism then so are $f$ and $h$.

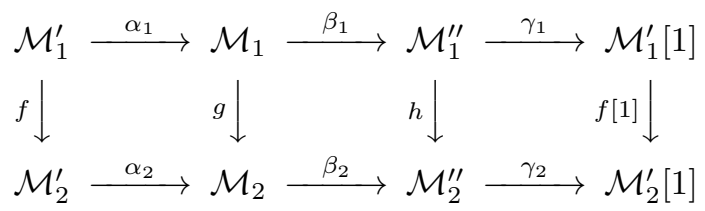

Proof. By assumption we have $\mathcal{M}_{1}^{\prime} \in{ }^{p} \mathrm{D}_{\mathrm{c}}^{\mathrm{b}}(\operatorname{Mod} \mathcal{A})^{\leq 0}$ and $\mathcal{M}_{2}^{\prime \prime}[i] \in{ }^{p} \mathrm{D}_{\mathrm{c}}^{\mathrm{b}}(\operatorname{Mod} \mathcal{A})^{\geq 1}$ for all $i \leq 0$. So by to Lemma $4.8(3)$ we get $\operatorname{Hom}_{\mathrm{D}(\operatorname{Mod} \mathcal{A})}\left(\mathcal{M}_{1}^{\prime}, \mathcal{M}_{2}^{\prime \prime}[i]\right)=0$ for $i \leq 0$. According to [BBD, Proposition 1.1.9] there exist unique morphisms $f$ and $h$ making the diagram commutative. The assertion about isomorphisms is also in BBD Proposition 1.1.9]. 
If $V \subset X$ is any open subset define $\left.\mathfrak{B}\right|_{V}:=\{U \in \mathfrak{B} \mid U \subset V\}$. Then $\left\{\mathfrak{T}_{U}\right\}_{\left.U \in \mathfrak{B}\right|_{V}}$ is a local collection of t-structures on the ringed space $\left(V,\left.\mathcal{A}\right|_{V}\right)$; hence using Definition 4.7 we get subcategories ${ }^{p} \mathrm{D}_{\mathrm{c}}^{\mathrm{b}}\left(\left.\operatorname{Mod} \mathcal{A}\right|_{V}\right)^{\star}$ of $\mathrm{D}_{\mathrm{c}}^{\mathrm{b}}\left(\left.\operatorname{Mod} \mathcal{A}\right|_{V}\right)$.

Lemma 4.11. Let $V \subset X$ be an open set, and let $V=\bigcup_{j} V_{j}$ be some open covering (where $V$ and $V_{j}$ are not necessarily in $\left.\mathfrak{B}\right)$. Write " $\star$ " for either " $\leq 0$ " or " $\geq 0$ ". Then the following are equivalent for $\mathcal{M} \in \mathrm{D}_{\mathrm{c}}^{\mathrm{b}}\left(\left.\operatorname{Mod} \mathcal{A}\right|_{V}\right)$.

(i) $\mathcal{M} \in{ }^{p} \mathrm{D}_{\mathrm{c}}^{\mathrm{b}}\left(\left.\operatorname{Mod} \mathcal{A}\right|_{V}\right)^{\star}$.

(ii) $\left.\mathcal{M}\right|_{V_{j}} \in{ }^{p} \mathrm{D}_{\mathrm{f}}^{\mathrm{b}}\left(\left.\operatorname{Mod} \mathcal{A}\right|_{V_{j}}\right)^{\star}$ for all $j$.

Proof. The implication (i) $\Rightarrow$ (ii) is immediate from Definition 4.7 For the reverse implication use condition $(\diamond)$ of Definition 4.6 twice with suitable $\mathfrak{B}$-coverings of $V$ and of $V_{j}$.

Lemma 4.12. Let $U \subset X$ be an open set, and let $U=\bigcup_{i=1}^{n} U_{i}$ be some covering by open sets $\left(U\right.$ and $U_{i}$ not necessarily in $\left.\mathfrak{B}\right)$. Let $\mathcal{M} \in \mathrm{D}_{\mathrm{c}}^{\mathrm{b}}\left(\left.\operatorname{Mod} \mathcal{A}\right|_{U}\right)$, and suppose there are distinguished triangles

$$
T_{i}=\left(\left.\mathcal{M}_{i}^{\prime} \stackrel{\alpha_{i}}{\longrightarrow} \mathcal{M}\right|_{U_{i}} \stackrel{\beta_{i}}{\longrightarrow} \mathcal{M}_{i}^{\prime \prime} \stackrel{\gamma_{i}}{\longrightarrow} \mathcal{M}_{i}^{\prime}[1]\right)
$$

in $\mathrm{D}_{\mathrm{c}}^{\mathrm{b}}\left(\left.\operatorname{Mod} \mathcal{A}\right|_{U_{i}}\right)$ with $\mathcal{M}_{i}^{\prime} \in{ }^{p} \mathrm{D}_{\mathrm{c}}^{\mathrm{b}}\left(\left.\operatorname{Mod} \mathcal{A}\right|_{U_{i}}\right) \leq 0$ and $\mathcal{M}_{i}^{\prime \prime} \in{ }^{p} \mathrm{D}_{\mathrm{c}}^{\mathrm{b}}\left(\left.\operatorname{Mod} \mathcal{A}\right|_{U_{i}}\right) \geq 1$. Then there exists a distinguished triangle

$$
\mathcal{M}^{\prime} \stackrel{\alpha}{\rightarrow} \mathcal{M} \stackrel{\beta}{\rightarrow} \mathcal{M}^{\prime \prime} \stackrel{\gamma}{\rightarrow} \mathcal{M}^{\prime}[1]
$$

in $\mathrm{D}_{\mathrm{c}}^{\mathrm{b}}\left(\left.\operatorname{Mod} \mathcal{A}\right|_{U}\right)$ whose restriction to $U_{i}$ is isomorphic to $T_{i}$.

Proof. First assume $n=2$. Denote by $g_{i}: U_{i} \rightarrow U$ the inclusions, for $i=1,2$. Also write $U_{(1,2)}:=U_{1} \cap U_{2}$ and $g_{(1,2)}: U_{(1,2)} \rightarrow U$. For any open immersion $g$ let $g$ ! be extension by zero, which is an exact functor.

The restriction of the triangles $T_{1}$ and $T_{2}$ to $U_{(1,2)}$ and Lemma4.10 give rise to an isomorphism $f:\left.\left.\mathcal{M}_{1}^{\prime}\right|_{U_{(1,2)}} \stackrel{\simeq}{\rightarrow} \mathcal{M}_{2}^{\prime}\right|_{U_{(1,2)}}$ in $\mathrm{D}_{\mathrm{c}}^{\mathrm{b}}\left(\left.\operatorname{Mod} \mathcal{A}\right|_{U_{(1,2)}}\right)$ satisfying $\alpha_{2} \circ f=\alpha_{1}$. Therefore we get a morphism

$$
\delta: g_{(1,2) !}\left(\left.\mathcal{M}_{1}^{\prime}\right|_{U_{(1,2)}}\right) \rightarrow g_{1 !} \mathcal{M}_{1}^{\prime} \oplus g_{2 !} \mathcal{M}_{2}^{\prime}
$$

in $\mathrm{D}^{\mathrm{b}}\left(\left.\operatorname{Mod} \mathcal{A}\right|_{U}\right)$, whose components are extensions by zero of the identity and $f$ respectively. (Note that the complexes $g_{1 !} \mathcal{M}_{1}^{\prime}$ etc. might not have cohomologies in C.)

Define $\mathcal{M}^{\prime}$ to be the cone of $\delta$. So there is a distinguished triangle

$$
g_{(1,2) !}\left(\left.\mathcal{M}_{1}^{\prime}\right|_{U_{(1,2)}}\right) \stackrel{\delta}{\rightarrow} g_{1 !} \mathcal{M}_{1}^{\prime} \oplus g_{2 !} \mathcal{M}_{2}^{\prime} \rightarrow \mathcal{M}^{\prime} \rightarrow g_{(1,2) !}\left(\left.\mathcal{M}_{1}^{\prime}\right|_{U_{(1,2)}}\right)[1]
$$

in $\mathrm{D}\left(\left.\operatorname{Mod} \mathcal{A}\right|_{U}\right)$. Applying the cohomological functor $\operatorname{Hom}_{\mathrm{D}\left(\left.\operatorname{Mod} \mathcal{A}\right|_{U}\right)}\left(-,\left.\mathcal{M}\right|_{U}\right)$ to this triangle we get an exact sequence

$$
\begin{aligned}
\operatorname{Hom}_{\mathrm{D}\left(\left.\operatorname{Mod} \mathcal{A}\right|_{U}\right)}\left(\mathcal{M}^{\prime},\left.\mathcal{M}\right|_{U}\right) & \rightarrow \operatorname{Hom}_{\mathrm{D}\left(\left.\operatorname{Mod} \mathcal{A}\right|_{U}\right)}\left(g_{1 !} \mathcal{M}_{1}^{\prime} \oplus g_{2 !} \mathcal{M}_{2}^{\prime},\left.\mathcal{M}\right|_{U}\right) \\
& \rightarrow \operatorname{Hom}_{\mathrm{D}\left(\left.\operatorname{Mod} \mathcal{A}\right|_{U}\right)}\left(g_{(1,2) !}\left(\left.\mathcal{M}_{1}^{\prime}\right|_{U_{(1,2)}}\right),\left.\mathcal{M}\right|_{U}\right) .
\end{aligned}
$$

The pair $\left(\alpha_{1},-\alpha_{2}\right)$ in the middle term goes to zero, and hence it comes from some morphism $\alpha:\left.\mathcal{M}^{\prime} \rightarrow \mathcal{M}\right|_{U}$. By construction the restriction $\left.\mathcal{M}^{\prime}\right|_{U_{i}} \cong \mathcal{M}_{i}^{\prime}$, so with the help of Lemma 4.11 we deduce that $\mathcal{M}^{\prime} \in{ }^{p} \mathrm{D}_{\mathrm{c}}^{\mathrm{b}}\left(\left.\operatorname{Mod} \mathcal{A}\right|_{U}\right) \leq 0$. Also the restriction of $\left.\mathcal{M}^{\prime} \stackrel{\alpha}{\rightarrow} \mathcal{M}\right|_{U}$ to $U_{i}$ is $\left.\mathcal{M}_{i}^{\prime} \stackrel{\alpha_{i}}{\longrightarrow} \mathcal{M}\right|_{U_{i}}$. 
Define $\mathcal{M}^{\prime \prime}$ to be the cone of $\alpha$. So we have a distinguished triangle

$$
T=\left(\left.\mathcal{M}^{\prime} \stackrel{\alpha}{\rightarrow} \mathcal{M}\right|_{U} \stackrel{\beta}{\rightarrow} \mathcal{M}^{\prime \prime} \stackrel{\gamma}{\rightarrow} \mathcal{M}^{\prime}[1]\right)
$$

in $\mathrm{D}^{\mathrm{b}}\left(\left.\operatorname{Mod} \mathcal{A}\right|_{U}\right)$. By Lemma 4.10 there is a (unique) isomorphism $\left.\mathcal{M}^{\prime \prime}\right|_{U_{i}} \stackrel{\simeq}{\longrightarrow}$ $\mathcal{M}_{i}^{\prime \prime}$ such that $\left.T\right|_{U_{i}} \stackrel{\simeq}{\rightarrow} T_{i}$ is an isomorphism of triangles. Therefore $\left.\mathcal{M}^{\prime \prime}\right|_{U_{i}} \in$ ${ }^{p} \mathrm{D}_{\mathrm{c}}^{\mathrm{b}}\left(\left.\operatorname{Mod} \mathcal{A}\right|_{U_{i}}\right) \geq 1$. Using Lemma 4.11 we see that $\mathcal{M}^{\prime \prime} \in{ }^{p} \mathrm{D}_{\mathrm{c}}^{\mathrm{b}}\left(\left.\operatorname{Mod} \mathcal{A}\right|_{U}\right)^{\geq 1}$.

When $n>2$ the statement follows from induction and the case $n=2$.

Theorem 4.14. Let $(X, \mathcal{A})$ be a ringed space with a local collection of $t$-structures $\left\{\mathfrak{T}_{U}\right\}_{U \in \mathfrak{B}}$. Assume $X$ is a noetherian topological space. Then the pair

$$
\left({ }^{p} \mathrm{D}_{\mathrm{c}}^{\mathrm{b}}(\operatorname{Mod} \mathcal{A}) \leq 0,{ }^{p} \mathrm{D}_{\mathrm{c}}^{\mathrm{b}}(\operatorname{Mod} \mathcal{A})^{\geq 0}\right)
$$

from Definition 4.7 is a $t$-structure on $\mathrm{D}_{\mathrm{c}}^{\mathrm{b}}(\operatorname{Mod} \mathcal{A})$.

Proof. Condition (i) of Definition 4.1 is trivially verified.

Let $\mathcal{M} \in{ }^{p} \mathrm{D}_{\mathrm{c}}^{\mathrm{b}}(\operatorname{Mod} \mathcal{A}) \leq 0$ and $\mathcal{N} \in{ }^{p} \mathrm{D}_{\mathrm{c}}^{\mathrm{b}}(\operatorname{Mod} \mathcal{A})^{\geq 1}$. So $\mathcal{N}[1] \in{ }^{p} \mathrm{D}_{\mathrm{c}}^{\mathrm{b}}(\operatorname{Mod} \mathcal{A})^{\geq 0}$, and by Lemma $4.8(3)$ with $i=-1$ we have $\operatorname{Hom}_{\mathrm{D}(\operatorname{Mod} \mathcal{A})}(\mathcal{M}, \mathcal{N})=0$. This verifies condition (ii).

It remains to prove condition (iii). Let a complex $\mathcal{M} \in \mathrm{D}_{\mathrm{c}}^{\mathrm{b}}(\operatorname{Mod} \mathcal{A})$ be given. Choose a covering $X=\bigcup_{i=1}^{n} U_{i}$ by open sets in $\mathfrak{B}$. For every $i$ the t-structure $\mathfrak{T}_{U_{i}}$ gives rise to a distinguished triangle

$$
S_{i}=\left(\left.\mathcal{M}_{i}^{\prime} \stackrel{\alpha_{i}}{\longrightarrow} \mathcal{M}\right|_{U_{i}} \stackrel{\beta_{i}}{\longrightarrow} \mathcal{M}_{i}^{\prime \prime} \stackrel{\gamma_{i}}{\longrightarrow} \mathcal{M}_{i}^{\prime}[1]\right)
$$

in $\mathrm{D}_{\mathrm{c}}^{\mathrm{b}}\left(\left.\operatorname{Mod} \mathcal{A}\right|_{U_{i}}\right)$ with $\mathcal{M}_{i}^{\prime} \in{ }^{p} \mathrm{D}_{\mathrm{c}}^{\mathrm{b}}\left(\left.\operatorname{Mod} \mathcal{A}\right|_{U_{i}} \leq 0\right.$ and $\mathcal{M}_{i}^{\prime \prime} \in{ }^{p} \mathrm{D}_{\mathrm{c}}^{\mathrm{b}}\left(\left.\operatorname{Mod} \mathcal{A}\right|_{U_{i}}\right)^{\geq 1}$. By Lemma 4.12 there is a triangle

$$
\mathcal{M}^{\prime} \stackrel{\alpha}{\rightarrow} \mathcal{M} \stackrel{\beta}{\rightarrow} \mathcal{M}^{\prime \prime} \stackrel{\gamma}{\rightarrow} \mathcal{M}[1]
$$

in $\mathrm{D}_{\mathrm{c}}^{\mathrm{b}}(\operatorname{Mod} \mathcal{A})$ whose restriction to each $U_{i}$ is isomorphic to $S_{i}$. Therefore by Lemma 4.11 one has $\mathcal{M}^{\prime} \in{ }^{p} \mathrm{D}_{\mathrm{c}}^{\mathrm{b}}(\operatorname{Mod} \mathcal{A}) \leq 0$ and $\mathcal{M}^{\prime \prime} \in{ }^{p} \mathrm{D}_{\mathrm{c}}^{\mathrm{b}}(\operatorname{Mod} \mathcal{A})^{\geq 1}$.

Theorem 4.15. Let $(X, \mathcal{A})$ be a ringed space with a local collection of $t$-structures $\left\{\mathfrak{T}_{U}\right\}_{U \in \mathfrak{B}}$. Assume $X$ is a noetherian topological space. For any open set $V \subset X$ let ${ }^{p} \mathrm{D}_{\mathrm{c}}^{\mathrm{b}}\left(\left.\operatorname{Mod} \mathcal{A}\right|_{V}\right)^{0}$ be the heart of the t-structure from Theorem 4.14. Then $V \mapsto$ ${ }^{p} \mathrm{D}_{\mathrm{c}}^{\mathrm{b}}\left(\left.\operatorname{Mod} \mathcal{A}\right|_{V}\right)^{0}$ is a stack of subcategories of $\mathrm{D}_{\mathrm{c}}^{\mathrm{b}}(\operatorname{Mod} \mathcal{A})$.

Proof. Axiom (a) follows from Lemma 4.11 Axiom (c) is Lemma 4.8 (2). Let us prove axiom (b). Suppose we are given open sets $V=\bigcup_{i \in I} V_{i} \subset X$, complexes $\mathcal{M}_{i} \in{ }^{p} \mathrm{D}_{\mathrm{c}}^{\mathrm{b}}\left(\left.\operatorname{Mod} \mathcal{A}\right|_{V_{i}}\right)^{0}$ and isomorphisms $\phi_{i, j}:\left.\left.\mathcal{M}_{i}\right|_{V_{i} \cap V_{j}} \rightarrow \mathcal{M}_{j}\right|_{V_{i} \cap V_{j}}$ satisfying the cocycle condition. Since $X$ is noetherian, and in view of axiom (c), we may assume $I=\{1, \ldots, n\}$. Let us define $W_{i}:=\bigcup_{j=1}^{i} V_{j}$. By induction on $i$ we will construct an object $\mathcal{N}_{i} \in{ }^{p} \mathrm{D}_{\mathrm{c}}^{\mathrm{b}}\left(\left.\operatorname{Mod} \mathcal{A}\right|_{W_{i}}\right)^{0}$ with isomorphisms $\psi_{i, j}:\left.\mathcal{N}_{i}\right|_{V_{j}} \stackrel{\simeq}{\rightarrow} \mathcal{M}_{j}$ for all $j \leq i$ that are compatible with the $\phi_{j, k}$. Then $\mathcal{M}:=\mathcal{N}_{n}$ will be the desired global object on $V=W_{n}$.

So assume $i<n$ and $\mathcal{N}_{i}$ has already been defined. For any $j \leq i$ we have an isomorphism

$$
\phi_{j, i+1} \circ \psi_{i, j}:\left.\left.\left.\mathcal{N}_{i}\right|_{V_{j} \cap V_{i+1}} \stackrel{\simeq}{\rightarrow} \mathcal{M}_{j}\right|_{V_{j} \cap V_{i+1}} \stackrel{\simeq}{\rightarrow} \mathcal{M}_{i+1}\right|_{V_{j} \cap V_{i+1}},
$$

and these satisfy the cocycle condition. According to Lemma 4.8 2 ) there is an isomorphism

$$
\psi_{i, i+1}:\left.\left.\mathcal{N}_{i}\right|_{W_{i} \cap V_{i+1}} \stackrel{\simeq}{\longrightarrow} \mathcal{M}_{i+1}\right|_{W_{i} \cap V_{i+1}}
$$


in ${ }^{p} \mathrm{D}_{\mathrm{c}}^{\mathrm{b}}\left(\left.\operatorname{Mod} \mathcal{A}\right|_{W_{i} \cap V_{i+1}}\right)^{0}$. Denote by $f_{i+1}: W_{i} \rightarrow W_{i+1}, g_{i+1}: V_{i+1} \rightarrow W_{i+1}$ and $h_{i+1}: W_{i} \cap V_{i+1} \rightarrow W_{i+1}$ the inclusions. Define $\mathcal{N}_{i+1} \in \mathrm{D}\left(\left.\operatorname{Mod} \mathcal{A}\right|_{W_{i+1}}\right)$ to be the cone of the morphism

$$
h_{(i+1) !}\left(\left.\mathcal{N}_{i}\right|_{W_{i} \cap V_{i+1}}\right) \stackrel{\left(\gamma, \psi_{i, i+1}\right)}{\longrightarrow} f_{(i+1) !} \mathcal{N}_{i} \oplus g_{(i+1) !} \mathcal{M}_{i+1}
$$

where $\gamma$ is the canonical morphism. We obtain a distinguished triangle

$$
h_{(i+1) !}\left(\left.\mathcal{N}_{i}\right|_{W_{i} \cap V_{i+1}}\right) \rightarrow f_{(i+1) !} \mathcal{N}_{i} \oplus g_{(i+1) !} \mathcal{M}_{i+1} \rightarrow \mathcal{N}_{i+1} \rightarrow h_{(i+1) !}\left(\left.\mathcal{N}_{i}\right|_{W_{i} \cap V_{i+1}}\right)[1]
$$

in $\mathrm{D}\left(\left.\operatorname{Mod} \mathcal{A}\right|_{W_{i+1}}\right)$. Upon restriction to $W_{i}$ we get an isomorphism $\left.\mathcal{N}_{i} \cong \mathcal{N}_{i+1}\right|_{W_{i}}$; and upon restriction to $V_{i+1}$ we get an isomorphism $\left.\mathcal{N}_{i+1}\right|_{V_{i+1}} \stackrel{\simeq}{\rightarrow} \mathcal{M}_{i+1}$ which we call $\psi_{i+1, i+1}$. Finally from Lemma 4.11 we see that $\mathcal{N}_{i+1} \in{ }^{p} \mathrm{D}_{\mathrm{c}}^{\mathrm{b}}\left(\left.\operatorname{Mod} \mathcal{A}\right|_{W_{i+1}}\right)^{0}$.

Remark 4.16. In $\mathrm{BBD}$ the authors considered t-structures on certain subcategories of $\mathrm{D}\left(\operatorname{Mod} \mathbb{k}_{X}\right)$, for a topological space $X$ and a constant sheaf of rings $\mathbb{k}_{X}$. Perverse t-structures on $\mathrm{D}_{\mathrm{c}}^{\mathrm{b}}(\operatorname{Mod} \mathcal{A})$ as above where $\mathcal{A}$ is "quasi-coherent" have only been considered recently; see $[\mathrm{Bz},[\mathrm{Br},[\mathrm{Ka}]$ and $[\mathrm{YZ} 4$.

\section{Differential Quasi-Coherent Ringed Schemes of Finite Type}

In this section all the pieces of our puzzle come together, and we prove the main result Theorem 0.3 - repeated here as Theorem [5.11 As before $\mathbb{k}$ denotes the base field. All schemes are over $\mathbb{k}$, all rings are $\mathbb{k}$-algebras, all bimodules are central over $\mathbb{k}$, and all homomorphisms are over $\mathbb{k}$.

Definition 5.1. Let $X$ be a finite type $\mathbb{k}$-scheme and let $\mathcal{A}$ be a quasi-coherent $\mathcal{O}_{X^{-}}$ ring. A differential quasi-coherent $\mathcal{O}_{X}$-filtration of finite type on $\mathcal{A}$ is an ascending filtration $F=\left\{F_{i} \mathcal{A}\right\}_{i \in \mathbb{Z}}$ by subsheaves with the following properties:

(i) Each $F_{i} \mathcal{A}$ is an $\mathcal{O}_{X}$-sub-bimodule of $\mathcal{A}$, quasi-coherent on both sides.

(ii) $F_{-1} \mathcal{A}=0$ and $\mathcal{A}=\bigcup F_{i} \mathcal{A}$.

(iii) $1 \in F_{0} \mathcal{A}$ and $\left(F_{i} \mathcal{A}\right) \cdot\left(F_{j} \mathcal{A}\right) \subset F_{i+j} \mathcal{A}$.

(iv) The graded sheaf of rings $\operatorname{gr}^{F} \mathcal{A}$ is an $\mathcal{O}_{X}$-algebra.

(v) The center $\mathrm{Z}\left(\mathrm{gr}^{F} \mathcal{A}\right)$ is a finite type quasi-coherent $\mathcal{O}_{X}$-algebra.

(vi) $\operatorname{gr}^{F} \mathcal{A}$ is a coherent $\mathrm{Z}\left(\operatorname{gr}^{F} \mathcal{A}\right)$-module.

By properties (i) and (iii) we get a ring homomorphism $\mathcal{O}_{X} \rightarrow \operatorname{gr}^{F} \mathcal{A}$. Property (iv) tells us that the image of $\mathcal{O}_{X}$ is inside $\mathrm{Z}\left(\operatorname{gr}^{F} \mathcal{A}\right)$. By (v-vi) we see that $\Gamma\left(U, \operatorname{gr}^{F} \mathcal{A}\right)$ is a noetherian ring for any affine open set $U$, so $(X, \mathcal{A})$ is a noetherian quasi-coherent ringed scheme.

Observe that the definition is left-right symmetric: if $\mathcal{A}$ is a differential quasicoherent $\mathcal{O}_{X}$-ring of finite type then so is $\mathcal{A}^{\text {op }}$. The name "differential filtration" signifies the resemblance to Grothendieck's definition of differential operators in EGA IV.

Definition 5.2. Let $X$ be a finite type $\mathbb{k}$-scheme. A differential quasi-coherent $\mathcal{O}_{X}$-ring of finite type is an $\mathcal{O}_{X}$-ring $\mathcal{A}$ that admits some differential quasi-coherent $\mathcal{O}_{X}$-filtration of finite type. The pair $(X, \mathcal{A})$ is then called a differential quasicoherent ringed $\mathbb{k}$-scheme finite type.

Let us recall a couple of definition from YY4.

Definition 5.3 (YZ4 Definition 2.1]). Suppose $C$ is a commutative $\mathbb{k}$-algebra and $A$ is a $C$-ring. A differential $C$-filtration on $A$ is a filtration $F=\left\{F_{i} A\right\}_{i \in \mathbb{Z}}$ with the following properties: 
(i) Each $F_{i} A$ is a $C$-sub-bimodule.

(ii) $F_{-1} A=0$ and $A=\bigcup F_{i} A$.

(iii) $1 \in F_{0} A$ and $F_{i} A \cdot F_{j} A \subset F_{i+j} A$.

(iv) The graded $\operatorname{ring} \operatorname{gr}^{F} A$ is a $C$-algebra.

A is called a differential $C$-ring if it admits some differential $C$-filtration.

Definition 5.4 (YZ4 Definition 2.2]). Let $C$ be a commutative noetherian $\mathbb{k}$ algebra, and let $A$ be a $C$-ring.

(1) A differential $C$-filtration of finite type on $A$ is a differential $C$-filtration $F=\left\{F_{i} A\right\}$ such that the graded $C$-algebra $\operatorname{gr}^{F} A$ is a finitely generated module over its center $\mathrm{Z}\left(\operatorname{gr}^{F} A\right)$, and $\mathrm{Z}\left(\operatorname{gr}^{F} A\right)$ is a finitely generated $C$ algebra.

(2) We say $A$ is a differential $C$-ring of finite type if it admits some differential $C$-filtration of finite type.

(3) If $A$ is a differential $\mathbb{k}$-ring of finite type then we also call $A$ a differential $\mathbb{k}$-algebra of finite type.

The next proposition says that a differential $C$-ring of finite type is just the ring theoretic counterpart of a differential quasi-coherent ringed $\mathbb{k}$-scheme finite type.

Proposition 5.5. Let $C$ be a noetherian $\mathbb{k}$-algebra and $U:=\operatorname{Spec} C$.

(1) Given a differential quasi-coherent $\mathcal{O}_{U}$-ring of finite type $\mathcal{A}$ the ring $A:=$ $\Gamma(U, \mathcal{A})$ is a differential $C$-ring of finite type.

(2) Given a differential $C$-ring of finite type $A$ there is a differential quasicoherent $\mathcal{O}_{U}$-ring of finite type $\mathcal{A}$, unique up to isomorphism, such that $\Gamma(U, \mathcal{A}) \cong A$ as $C$-rings.

Proof. The proof of (1) is straightforward. For (2) use [YZ4 Proposition 5.17 and Corollary 5.13]. As filtration on $\mathcal{A}$ we may take $F_{i} \mathcal{A}:=\mathcal{O}_{U} \otimes_{C} F_{i} A$ where $\left\{F_{i} A\right\}$ is any differential $C$-filtration of finite type on $A$.

Proposition 5.6. Suppose $(X, \mathcal{A})$ and $(Y, \mathcal{B})$ are two differential quasi-coherent ringed schemes of finite type over $\mathbb{k}$. Then the product $(X \times Y, \mathcal{A} \otimes \mathcal{B})$ exists (cf. Definition 4.11), and it too is a differential quasi-coherent ringed scheme of finite type over $\mathbb{k}$.

Proof. Let $A, B, C$ be as in condition (ii) of Theorem 1.10 Then [YZ4, Proposition 2.9 ] tells us that $A \otimes B$ is a differential $C$-ring of finite type. Using [YZ4 Proposition 5.14 and Corollary 5.13] we see that condition (ii) of Theorem [1.10 is satisfied. Therefore the product $(X \times Y, \mathcal{A} \otimes \mathcal{B})$ exists.

Given differential filtrations of $\mathcal{A}$ and $\mathcal{B}$ one can construct a filtration on $\mathcal{A} \otimes \mathcal{B}$ by gluing together the affine filtrations described in the proof of YZ4 Proposition 2.9].

Definition 5.7. Let $A$ be a noetherian $\mathbb{k}$-algebra with rigid dualizing complex $R_{A}$, and let

$$
\mathrm{D}:=\operatorname{RHom}_{A}\left(-, R_{A}\right): \mathrm{D}_{\mathrm{f}}^{\mathrm{b}}(\operatorname{Mod} A) \rightarrow \mathrm{D}_{\mathrm{f}}^{\mathrm{b}}\left(\operatorname{Mod} A^{\mathrm{op}}\right)
$$

be the induced duality. The rigid perverse t-structure on $\mathrm{D}_{\mathrm{f}}^{\mathrm{b}}(\operatorname{Mod} A)$ is defined by

$$
\begin{aligned}
& { }^{p} \mathrm{D}_{\mathrm{f}}^{\mathrm{b}}(\operatorname{Mod} A)^{\leq 0}:=\left\{M \in \mathrm{D}_{\mathrm{f}}^{\mathrm{b}}(\operatorname{Mod} A) \mid \mathrm{H}^{i} \mathrm{D} M=0 \text { for all } i<0\right\}, \\
& { }^{p} \mathrm{D}_{\mathrm{f}}^{\mathrm{b}}(\operatorname{Mod} A)^{\geq 0}:=\left\{M \in \mathrm{D}_{\mathrm{f}}^{\mathrm{b}}(\operatorname{Mod} A) \mid \mathrm{H}^{i} \mathrm{D} M=0 \text { for all } i>0\right\} .
\end{aligned}
$$

An object $M \in{ }^{p} \mathrm{D}_{\mathrm{f}}^{\mathrm{b}}(\operatorname{Mod} A)^{0}$ is called a perverse $A$-module. 
The rigid perverse t-structure on $\mathrm{D}_{\mathrm{f}}^{\mathrm{b}}(\operatorname{Mod} A)$ is of course dual to the standard t-structure on $\mathrm{D}_{\mathrm{f}}^{\mathrm{b}}\left(\operatorname{Mod} A^{\mathrm{op}}\right)$, via the duality $\mathrm{D}$.

Suppose $(X, \mathcal{A})$ is a differential quasi-coherent ringed scheme of finite type over $\mathbb{k}$. By Proposition $5.5(1)$, for every affine open set $U \subset X$ the $\mathbb{k}$-algebra $\Gamma(U, \mathcal{A})$ is a differential $\mathbb{k}$-algebra of finite type. Hence according to $[$ YZ4 Theorem 8.1] the algebra $\Gamma(U, \mathcal{A})$ has a rigid dualizing complex, and the rigid perverse t-structure on $\mathrm{D}_{\mathrm{f}}^{\mathrm{b}}(\operatorname{Mod} \mathcal{A})$ exists.

Definition 5.8. Let $(X, \mathcal{A})$ be a differential quasi-coherent ringed scheme of finite type over $\mathbb{k}$. Taking " $\star$ " to be either " $\leq 0$ ", " $\geq 0$ " or " 0 ", we define classes of objects

$$
{ }^{p} \mathrm{D}_{\mathrm{c}}^{\mathrm{b}}(\operatorname{Mod} \mathcal{A})^{\star}:=\left\{\mathcal{M} \in \mathrm{D}_{\mathrm{c}}^{\mathrm{b}}(\operatorname{Mod} \mathcal{A}) \mid \operatorname{R\Gamma }(U, \mathcal{M}) \in{ }^{p} \mathrm{D}_{\mathrm{f}}^{\mathrm{b}}(\operatorname{Mod} \Gamma(U, \mathcal{A}))^{\star}\right.
$$

for all affine open sets $U\}$.

Theorem 5.9. Let $(X, \mathcal{A})$ be a differential quasi-coherent ringed scheme of finite type over $\mathbb{k}$. Then:

(1) The pair

$$
\left({ }^{p} \mathrm{D}_{\mathrm{c}}^{\mathrm{b}}(\operatorname{Mod} \mathcal{A})^{\leq 0},{ }^{p} \mathrm{D}_{\mathrm{c}}^{\mathrm{b}}(\operatorname{Mod} \mathcal{A})^{\geq 0}\right)
$$

is a t-structure on $\mathrm{D}_{\mathrm{c}}^{\mathrm{b}}(\operatorname{Mod} \mathcal{A})$.

(2) The assignment $V \mapsto{ }^{p} \mathrm{D}_{\mathrm{c}}^{\mathrm{b}}\left(\left.\operatorname{Mod} \mathcal{A}\right|_{V}\right)^{0}$, for $V \subset X$ open, is a stack of abelian categories on $X$.

Proof. For an affine open set $U \subset X$ let

$$
\mathfrak{T}_{U}:=\left({ }^{p} \mathrm{D}_{\mathrm{c}}^{\mathrm{b}}\left(\left.\operatorname{Mod} \mathcal{A}\right|_{U}\right) \leq 0,{ }^{p} \mathrm{D}_{\mathrm{c}}^{\mathrm{b}}\left(\left.\operatorname{Mod} \mathcal{A}\right|_{U}\right) \geq 0\right) .
$$

By Theorems 4.14 and 4.15 it suffices to prove that $\left\{\mathfrak{T}_{U}\right\}$ is a local collection of t-structures on $(X, \mathcal{A})$. Namely, given an affine open set $U$, a complex $\mathcal{M} \in$ $\mathrm{D}_{\mathrm{c}}^{\mathrm{b}}\left(\left.\operatorname{Mod} \mathcal{A}\right|_{U}\right)$, and an affine open covering $U=\bigcup U_{k}$, one has $\mathcal{M} \in{ }^{p} \mathrm{D}_{\mathrm{c}}^{\mathrm{b}}\left(\left.\operatorname{Mod} \mathcal{A}\right|_{U}\right)^{\star}$ if and only if $\left.\mathcal{M}\right|_{U_{k}} \in{ }^{p} \mathrm{D}_{\mathrm{c}}^{\mathrm{b}}\left(\left.\operatorname{Mod} \mathcal{A}\right|_{U_{k}}\right)^{\star}$ for all $k$.

Write $A:=\Gamma(U, \mathcal{A}), M:=\operatorname{R} \Gamma(U, \mathcal{M}), A_{k}:=\Gamma\left(U_{k}, \mathcal{A}\right)$ and $M_{k}:=\operatorname{R} \Gamma\left(U_{k}, \mathcal{M}\right)$. We must show that $M \in{ }^{p} \mathrm{D}_{\mathrm{f}}^{\mathrm{b}}(\operatorname{Mod} A)^{\star}$ if and only if $M_{k} \in{ }^{p} \mathrm{D}_{\mathrm{f}}^{\mathrm{b}}\left(\operatorname{Mod} A_{k}\right)^{\star}$ for all $k$.

Denote by $R_{A}$ and $R_{A_{k}}$ the rigid dualizing complexes of the rings $A$ and $A_{k}$ respectively. By YZ4 Corollary 5.20] $A_{k}$ is a localization of $A$. According to $[\mathrm{YZ4}$, Theorem 8.14 and Propositions 5.21 and 5.17] each $A$-bimodule $\mathrm{H}^{i} R_{A}$ is evenly localizable to $A_{k}$. Hence YZ4 Theorem 6.2] tells us that $R_{A_{k}} \cong R_{A} \otimes_{A} A_{k}$ in $\mathrm{D}\left(\operatorname{Mod} A \otimes A_{k}^{\mathrm{op}}\right)$, and likewise $R_{A_{k}} \cong A_{k} \otimes_{A} R_{A}$ in $\mathrm{D}\left(\operatorname{Mod} A_{k} \otimes A^{\mathrm{op}}\right)$.

Define complexes $N:=\operatorname{RHom}_{A}\left(M, R_{A}\right)$ and $N_{k}:=\operatorname{RHom}_{A_{k}}\left(M_{k}, R_{A_{k}}\right)$. According to Lemma 1.19 we have $M_{k} \cong A_{k} \otimes_{A} M$. By [YZ3, Lemma 3.7]

$$
\begin{aligned}
N \otimes_{A} A_{k} & \cong \operatorname{RHom}_{A}\left(M, R_{A} \otimes_{A} A_{k}\right) \cong \operatorname{RHom}_{A}\left(M, R_{A_{k}}\right) \\
& \cong \operatorname{RHom}_{A_{k}}\left(A_{k} \otimes_{A} M, R_{A_{k}}\right) \cong \operatorname{RHom}_{A_{k}}\left(M_{k}, R_{A_{k}}\right)=N_{k} .
\end{aligned}
$$

Let us consider the case where " $\star$ " is " $\geq$ ". Suppose $M \in{ }^{p} \mathrm{D}_{\mathrm{f}}^{\mathrm{b}}(\operatorname{Mod} A) \geq 0$. By definition of the perverse rigid t-structure we have $N \in \mathrm{D}_{\mathrm{f}}^{\mathrm{b}}\left(\operatorname{Mod} A^{\mathrm{op}}\right) \leq 0$, i.e. $\mathrm{H}^{j} N=0$ for all $j>0$. Hence $\mathrm{H}^{j} N_{k} \cong\left(\mathrm{H}^{j} N\right) \otimes_{A} A_{k}=0$, implying that $M_{k} \in \mathrm{D}_{\mathrm{f}}^{\mathrm{b}}\left(\operatorname{Mod} A_{k}\right) \geq 0$.

Conversely suppose $M_{k} \in{ }^{p} \mathrm{D}_{\mathrm{f}}^{\mathrm{b}}\left(\operatorname{Mod} A_{k}\right) \geq 0$ for all $k$. We may assume that $U=$ $\bigcup_{k=1}^{n} U_{k}$. Let $C:=\Gamma\left(U, \mathcal{O}_{X}\right)$ and $C_{k}:=\Gamma\left(U_{k}, \mathcal{O}_{X}\right)$. The ring homomorphism $C \rightarrow \prod_{k=1}^{n} C_{k}$ is faithfully flat (cf. YZ4, Proposition 5.6]). Applying $A \otimes_{C}-$ it follows that $A \rightarrow \prod_{k=1}^{n} A_{k}$ is faithfully flat (on both sides), so we get an injection

$$
\mathrm{H}^{j} N \hookrightarrow \bigoplus_{k}\left(\mathrm{H}^{j} N\right) \otimes_{A} A_{k}
$$


As above we conclude that $N \in \mathrm{D}_{\mathrm{f}}^{\mathrm{b}}\left(\operatorname{Mod} A^{\mathrm{op}}\right) \leq 0$, and hence $M \in{ }^{p} \mathrm{D}_{\mathrm{f}}^{\mathrm{b}}(\operatorname{Mod} A) \geq 0$.

The case where " $\star$ " is " $\leq 0$ " is handled similarly. The only difference is that we have to verify the vanishing of $\mathrm{H}^{j} N$ and $\mathrm{H}^{j} N_{k}$ for $j<0$.

Definition 5.10. The t-structure on $\mathrm{D}_{\mathrm{c}}^{\mathrm{b}}(\operatorname{Mod} \mathcal{A})$ in Theorem[5.9 is called the rigid perverse $t$-structure. An object $\mathcal{M} \in{ }^{p} \mathrm{D}_{\mathrm{c}}^{\mathrm{b}}(\operatorname{Mod} \mathcal{A})^{0}$ is called a perverse coherent $\mathcal{A}$-module.

Theorem 5.11. Let $(X, \mathcal{A})$ be a separated differential quasi-coherent ringed scheme of finite type over $\mathbb{k}$. Then $\mathcal{A}$ has a rigid dualizing complex $\left(\mathcal{R}_{\mathcal{A}}, \boldsymbol{\rho}\right)$. It is unique up to a unique isomorphism in $\mathrm{D}_{\mathrm{c}}^{\mathrm{b}}\left(\operatorname{Mod} \mathcal{A}^{\mathrm{e}}\right)$.

Proof. By Proposition [5.6 the product $\left(X^{2}, \mathcal{A}^{\mathrm{e}}\right)$ is also a separated differential quasi-coherent ringed scheme of finite type over $\mathbb{k}$. Hence by Theorem 5.9 we obtain the rigid perverse t-structure on $\mathrm{D}_{\mathrm{c}}^{\mathrm{b}}\left(\operatorname{Mod} \mathcal{A}^{\mathrm{e}}\right)$, and there is a stack of abelian categories $W \mapsto{ }^{p} \mathrm{D}_{\mathrm{c}}^{\mathrm{b}}\left(\left.\operatorname{Mod} \mathcal{A}^{\mathrm{e}}\right|_{W}\right)^{0}$ on $X^{2}$.

Let us choose, for ease of notation, an indexing $\left\{U_{i}\right\}$ of the set Aff $X$ of affine open sets of $X$. For any index $i$ let $A_{i}:=\Gamma\left(U_{i}, \mathcal{A}\right)$, which is a differential $\mathbb{k}$-algebra of finite type. Then $A_{i}$ has a rigid dualizing complex $\left(R_{i}, \rho_{i}\right)$. By YZ4, Theorem 8.9] the complex $R_{i}$ is a perverse bimodule, i.e. $R_{i} \in{ }^{p} D_{\mathrm{f}}^{\mathrm{b}}\left(\operatorname{Mod} A_{i}^{\mathrm{e}}\right)^{0}$.

For a pair of indices $i, j$ let $A_{(i, j)}:=\Gamma\left(U_{i} \cap U_{j}, \mathcal{A}\right)$. Define

$$
R_{i \rightarrow(i, j)}:=A_{(i, j)} \otimes_{A_{i}} R_{i} \otimes_{A_{i}} A_{(i, j)} \in \mathrm{D}\left(\operatorname{Mod} A_{(i, j)}^{\mathrm{e}}\right),
$$

and let $\mathrm{q}_{A_{(i, j)} / A_{i}}: R_{A_{i}} \rightarrow R_{i \rightarrow(i, j)}$ be the morphism with formula $r \mapsto 1 \otimes r \otimes 1$. According to [YZ4, Theorem 6.2] the complex $R_{i \rightarrow(i, j)}$ is dualizing over the ring $A_{(i, j)}$, and it has a unique rigidifying isomorphism $\rho_{i \rightarrow(i, j)}$ such that $\mathrm{q}_{A_{(i, j)} / A_{i}}$ is a rigid localization morphism. Likewise we obtain a rigid dualizing complex $\left(R_{j \rightarrow(i, j)}, \rho_{j \rightarrow(i, j)}\right)$. By [YZ2, Corollary 4.3] there is a unique isomorphism

$$
\phi_{(i, j)}: R_{i \rightarrow(i, j)} \stackrel{\simeq}{\rightarrow} R_{j \rightarrow(i, j)}
$$

in $\mathrm{D}\left(\operatorname{Mod} A_{(i, j)}^{\mathrm{e}}\right)$ that's a rigid trace. The isomorphisms $\phi_{(i, j)}$ will then satisfy the cocycle condition in $\mathrm{D}\left(\operatorname{Mod} A_{(i, j, k)}^{\mathrm{e}}\right)$, where $A_{(i, j, k)}:=\Gamma\left(U_{i} \cap U_{j} \cap U_{k}, \mathcal{A}\right)$ for a triple intersection.

Now consider the affine ringed scheme $\left(U_{i}^{2},\left.\mathcal{A}^{\mathrm{e}}\right|_{U_{i}^{2}}\right)$. Let us denote by $\mathcal{R}_{i} \in$ $\mathrm{D}_{\mathrm{c}}^{\mathrm{b}}\left(\left.\operatorname{Mod} \mathcal{A}^{\mathrm{e}}\right|_{U_{i}^{2}}\right)$ the sheafification of $R_{i}$. By definition of the rigid perverse $\mathrm{t}$ structure on $\mathrm{D}_{\mathrm{c}}^{\mathrm{b}}\left(\left.\operatorname{Mod} \mathcal{A}^{\mathrm{e}}\right|_{U_{i}^{2}}\right)$ we have $\mathcal{R}_{i} \in{ }^{p} \mathrm{D}_{\mathrm{c}}^{\mathrm{b}}\left(\left.\operatorname{Mod} \mathcal{A}^{\mathrm{e}}\right|_{U_{i}^{2}}\right)^{0}$. By Lemma 1.19 we obtain induced isomorphisms

$$
\phi_{(i, j)}:\left.\left.\mathcal{R}_{i}\right|_{U_{i}^{2} \cap U_{j}^{2}} \stackrel{\simeq}{\rightarrow} \mathcal{R}_{j}\right|_{U_{i}^{2} \cap U_{j}^{2}}
$$

in ${ }^{p} \mathrm{D}_{\mathrm{c}}^{\mathrm{b}}\left(\left.\operatorname{Mod} \mathcal{A}^{\mathrm{e}}\right|_{U_{i}^{2} \cap U_{j}^{2}}\right)^{0}$, and these satisfy the cocycle condition on triple intersections. Let $V:=X^{2}-\Delta(X)$. Since $\left.\mathcal{R}_{i}\right|_{U_{i}^{2} \cap V}=0$ we have sufficient gluing data corresponding to the open covering $X^{2}=\left(\bigcup_{i} U_{i}^{2}\right) \cup V$, and by Theorem [5.9 we deduce the existence and uniqueness of a global complex $\mathcal{R}_{\mathcal{A}} \in{ }^{p} \mathrm{D}_{\mathrm{c}}^{\mathrm{b}}\left(\operatorname{Mod} \mathcal{A}^{\mathrm{e}}\right)^{0}$ together with isomorphisms $\phi_{i}:\left.\mathcal{R}_{\mathcal{A}}\right|_{U_{i}} \stackrel{\simeq}{\rightarrow} \mathcal{R}_{i}$. By Theorem 3.4 and Proposition 3.3 $\mathcal{R}_{\mathcal{A}}$ is a local dualizing complex over $\mathcal{A}$. And by construction $\mathcal{R}_{\mathcal{A}}$ comes equipped with a collection $\boldsymbol{\rho}=\left\{\rho_{i}\right\}$ of rigidifying isomorphisms that is compatible with the sheaf structure. 
Finite morphisms between ringed schemes were defined in Definition 3.9] A ring homomorphism $A \rightarrow B$ is called centralizing if $B=\sum A b_{i}$ for elements $b_{i} \in B$ that commute with $A$.

Definition 5.12. Let $f:(Y, \mathcal{B}) \rightarrow(X, \mathcal{A})$ be a finite morphism between two noetherian quasi-coherent ringed schemes over $\mathbb{k}$. We say $f$ is finite centralizing if for any affine open set $U \subset X$ the finite ring homomorphism $f^{*}: \Gamma(U, \mathcal{A}) \rightarrow \Gamma\left(U, f_{*} \mathcal{B}\right)$ is centralizing.

Example 5.13. Suppose $\mathcal{I} \subset \mathcal{A}$ is a coherent sheaf of (two-sided) ideals, and define $\mathcal{B}:=\mathcal{A} / \mathcal{I}$. Then $(X, \mathcal{B})$ is a differential quasi-coherent ringed scheme of finite type over $\mathbb{k}$, and $(X, \mathcal{B}) \rightarrow(X, \mathcal{A})$ is a finite centralizing morphism.

Proposition 5.14. Let $f:(Y, \mathcal{B}) \rightarrow(X, \mathcal{A})$ be a finite centralizing morphism between two separated differential quasi-coherent ringed schemes of finite type over $\mathbb{k}$. Consider the the rigid perverse $t$-structures on these ringed schemes.

(1) Let $\mathcal{M} \in \mathrm{D}_{\mathrm{c}}^{\mathrm{b}}(\operatorname{Mod} \mathcal{B})$. Then $\mathcal{M} \in{ }^{p} \mathrm{D}_{\mathrm{c}}^{\mathrm{b}}(\operatorname{Mod} \mathcal{B})^{0}$ if and only if $\mathrm{R} f_{*} \mathcal{M} \in$ ${ }^{p} \mathrm{D}_{\mathrm{c}}^{\mathrm{b}}(\operatorname{Mod} \mathcal{A})^{0}$.

(2) Assume $f^{*}: \mathcal{A} \rightarrow f_{*} \mathcal{B}$ is surjective. Then the functor

$$
\mathrm{R} f_{*}:{ }^{p} \mathrm{D}_{\mathrm{c}}^{\mathrm{b}}(\operatorname{Mod} \mathcal{B})^{0} \rightarrow{ }^{p} \mathrm{D}_{\mathrm{c}}^{\mathrm{b}}(\operatorname{Mod} \mathcal{A})^{0}
$$

is fully faithful.

Proof. (1) Let $U \subset X$ be an affine open set and $V:=f^{-1}(U)$. Define $A:=\Gamma(U, \mathcal{A})$, $B:=\Gamma(V, \mathcal{B})$ and $M:=\mathrm{R} \Gamma(V, \mathcal{M})$. By Lemma YZ5, Lemma 6.12] it is enough to show that $M \in{ }^{p} \mathrm{D}_{\mathrm{c}}^{\mathrm{b}}(\operatorname{Mod} B)^{0}$ if and only if $\operatorname{rest}_{B / A} M \in{ }^{p} \mathrm{D}_{\mathrm{c}}^{\mathrm{b}}(\operatorname{Mod} A)^{0}$. This is done in YYZ4 Proposition 8.3(1)].

(2) This follows from Theorem [5.9 and [YZ4, Proposition 5.3(2)].

Rigid trace morphisms between rigid dualizing complexes were defined in Definition 3.11

Theorem 5.15. Let $(X, \mathcal{A})$ and $(Y, \mathcal{B})$ be two separated differential quasi-coherent ringed schemes of finite type over $\mathbb{k}$, and let $f:(Y, \mathcal{B}) \rightarrow(X, \mathcal{A})$ be a finite centralizing morphism. Then there exists a unique rigid trace

$$
\operatorname{Tr}_{f}: \mathrm{R} f_{*}^{\mathrm{e}} \mathcal{R}_{\mathcal{B}} \rightarrow \mathcal{R}_{\mathcal{A}} .
$$

Proof. The morphism $f^{\mathrm{e}}:\left(Y^{2}, \mathcal{B}^{\mathrm{e}}\right) \rightarrow\left(X^{2}, \mathcal{A}^{\mathrm{e}}\right)$ is also finite centralizing, so by Proposition [5.14 we get $\mathrm{R} f_{*}^{\mathrm{e}} \mathcal{R}_{\mathcal{B}} \in{ }^{p} \mathrm{D}_{\mathrm{c}}^{\mathrm{b}}\left(\operatorname{Mod} \mathcal{A}^{\mathrm{e}}\right)^{0}$. Hence by Theorem [5.9] any morphism $\mathrm{R} f_{*}^{\mathrm{e}} \mathcal{R}_{\mathcal{B}} \rightarrow \mathcal{R}_{\mathcal{A}}$ is determined locally.

Choose an affine open covering $X=\bigcup_{i=1}^{n} U_{i}$ and let $A_{i}:=\Gamma\left(U_{i}, \mathcal{A}\right)$ and $B_{i}:=$ $\Gamma\left(f^{-1}\left(U_{i}\right), \mathcal{B}\right) \cong \Gamma\left(U_{i}, f_{*} \mathcal{B}\right)$. Since $A_{i} \rightarrow B_{i}$ is a finite centralizing homomorphism, YZ4 Proposition 8.2] asserts the existence of the trace $\operatorname{Tr}_{A_{i} / B_{i}}: R_{B_{i}} \rightarrow R_{A_{i}}$ in $\mathrm{D}_{\mathrm{f}}^{\mathrm{b}}\left(\operatorname{Mod} A^{\mathrm{e}}\right)$. The uniqueness of $\operatorname{Tr}_{A_{i} / B_{i}}$ is always true. By Corollary 1.18 we get a morphism

in ${ }^{p} \mathrm{D}_{\mathrm{c}}^{\mathrm{b}}\left(\left.\operatorname{Mod}\left(\mathcal{A}^{\mathrm{e}}\right)\right|_{U_{i}^{2}}\right)^{0}$.

$$
\operatorname{Tr}_{i}:\left.\left.\left(\mathrm{R} f_{*}^{\mathrm{e}} \mathcal{R}_{\mathcal{B}}\right)\right|_{U_{i}^{2}} \rightarrow \mathcal{R}_{\mathcal{A}}\right|_{U_{i}^{2}}
$$

By [YZ4, Proposition 6.3] the rigid trace localizes. Therefore the morphisms $\operatorname{Tr}_{i}$ coincide on intersections $U_{i}^{2} \cap U_{j}^{2}$. Both $\mathrm{R} f_{*}^{\mathrm{e}} \mathcal{R}_{\mathcal{B}}$ and $\mathcal{R}_{\mathcal{A}}$ are supported on the diagonal $\Delta(X)$. Therefore we have gluing data for a global morphism

$$
\operatorname{Tr}_{f}: \mathrm{R} f_{*}^{\mathrm{e}} \mathcal{R}_{\mathcal{B}} \rightarrow \mathcal{R}_{\mathcal{A}}
$$


in ${ }^{p} \mathrm{D}_{\mathrm{c}}^{\mathrm{b}}\left(\operatorname{Mod} \mathcal{A}^{\mathrm{e}}\right)^{0}$ as required.

The uniqueness of $\operatorname{Tr}_{f}$ is a consequence of the fact that ${ }^{p} D_{\mathrm{c}}^{\mathrm{b}}\left(\operatorname{Mod} \mathcal{A}^{\mathrm{e}}\right)^{0}$ is a stack and the uniqueness of the traces $\operatorname{Tr}_{i}$.

Example 5.16. If $X$ is a finite type $\mathbb{k}$-scheme and $\mathcal{A}=\mathcal{O}_{X}$ then conditions (i)(iii) in Theorem 5.9 hold. Hence $\mathrm{D}_{\mathrm{c}}^{\mathrm{b}}\left(\operatorname{Mod} \mathcal{O}_{X}\right)$ has the rigid perverse t-structure. In Section $\left[6\right.$ we show that the perverse coherent $\mathcal{O}_{X}$-modules are nothing but the Cohen-Macaulay complexes in $\mathrm{D}_{\mathrm{c}}^{\mathrm{b}}(\operatorname{Mod} \mathcal{A})$.

Remark 5.17. Suppose $(X, \mathcal{A})$ is a noetherian quasi-coherent ringed scheme. In his recent paper [Ka] Kashiwara proves that any bounded filtration of $X$ by families of supports induces a perverse t-structure on $\mathrm{D}_{\mathrm{qc}}^{\mathrm{b}}(\operatorname{Mod} \mathcal{A})$. In particular this is true for the filtration by codimension (coniveau) $\mathfrak{S}=\left\{\mathfrak{S}^{d}\right\}$.

When $\mathbb{k}:=\mathbb{C}, X$ is smooth and $\mathcal{A}:=\mathcal{D}_{X}$, Kashiwara proves that the t-structure induced by $\mathfrak{S}$ on the category $\mathrm{D}_{\mathrm{rh}}^{\mathrm{b}}\left(\operatorname{Mod} \mathcal{D}_{X}\right)$ of regular holonomic complexes is dual to the standard t-structure on $\mathrm{D}_{\mathrm{c}}^{\mathrm{b}}\left(\operatorname{Mod} \mathbb{C}_{X_{\mathrm{an}}}\right)$, via the Riemann-Hilbert correspondence $\mathrm{R} \mathcal{H} \operatorname{om}_{\mathcal{D}_{X}}\left(-, \mathcal{O}_{X_{\text {an }}}\right)$.

On the other hand if $\mathcal{A}$ is a coherent $\mathcal{O}_{X}$-algebra the t-structure induced by $\mathfrak{S}$ on $\mathrm{D}_{\mathrm{c}}^{\mathrm{b}}(\operatorname{Mod} \mathcal{A})$ is the rigid perverse t-structure, as can be seen from [YZ4, Theorem $0.6]$.

\section{Examples and Complements}

In this section we present several examples of differential quasi-coherent ringed schemes of finite type over $\mathbb{k}$ and their rigid dualizing complexes, including the the commutative case $\mathcal{A}=\mathcal{O}_{X}$. Finally we discuss in detail the ringed scheme $\left(\mathbf{A}^{1}, \mathcal{D}_{\mathbf{A}^{1}}\right)$ and some of its partial compactifications.

Let $X$ be a separated $\mathbb{k}$-scheme, and consider the diagonal embedding $\Delta$ : $X \rightarrow X^{2}$. A central rigid dualizing complex over $X$ is a pair $\left(\mathcal{R}_{X}, \boldsymbol{\rho}_{X}\right)$, where $\mathcal{R}_{X} \in \mathrm{D}_{\mathrm{c}}^{\mathrm{b}}\left(\operatorname{Mod} \mathcal{O}_{X}\right)$ and $\left(\mathrm{R} \Delta_{*} \mathcal{R}_{X}, \boldsymbol{\rho}_{X}\right)$ is a rigid dualizing complex in the sense of Definition 3.7

Proposition 6.1. The scheme $X$ admits a central rigid dualizing complex, which is unique up to a unique isomorphism in $\mathrm{D}\left(\operatorname{Mod} \mathcal{O}_{X}\right)$.

Proof. See YZ5, Theorem 0.1]. This can also be proved with the methods of Section [5]

In case the structural morphism $\pi: X \rightarrow$ Spec $\mathbb{k}$ is embeddable then there is an isomorphism $\mathcal{R}_{X} \cong \pi^{!} \mathbb{k}$, where $\pi^{!}$is the twisted inverse image from [RD]; see [YZ5] Proposition 6.18].

Recall that a complex $\mathcal{M} \in \mathrm{D}^{\mathrm{b}}\left(\operatorname{Mod} \mathcal{O}_{X}\right)$ is called Cohen-Macaulay (with respect to the filtration by dimension) if for every point $x$ the local cohomologies $\mathrm{H}_{x}^{i} \mathcal{M}$ all vanish except for $i=-\operatorname{dim} \overline{\{x\}}$. Equivalently, $\mathcal{M}$ is Cohen-Macaulay if there is an isomorphism $\mathcal{M} \cong \mathrm{E} \mathcal{M}$ in $\mathrm{D}\left(\operatorname{Mod} \mathcal{O}_{X}\right)$, where $\mathrm{E} \mathcal{M}$ is the Cousin complex. See [RD, Section IV.3] or [YZ3, Theorem 2.11].

Theorem 6.2. Let $X$ be a separated finite type scheme over $\mathbb{k}$, let $\mathcal{R}_{X}$ be the central rigid dualizing complex of $X$, and let $\mathrm{D}_{X}$ be the auto-duality functor $\mathrm{R} \mathcal{H} m_{\mathcal{O}_{X}}\left(-, \mathcal{R}_{X}\right)$. Then the following conditions are equivalent for $\mathcal{M} \in \mathrm{D}_{\mathrm{c}}^{\mathrm{b}}\left(\operatorname{Mod} \mathcal{O}_{X}\right)$.

(i) $\mathcal{M}$ is a perverse coherent sheaf (for the rigid perverse $t$-structure).

(ii) $\mathrm{D} \mathcal{M}$ is a coherent sheaf, i.e. $\mathrm{H}^{i} \mathrm{D} \mathcal{M}=0$ for all $i \neq 0$. 
(iii) $\mathcal{M}$ is a Cohen-Macaulay complex.

Proof. All three conditions can be checked locally, so we may assume $X$ is affine. Then (i) and (ii) are equivalent by definition.

Since the dualizing complex $\mathcal{R}_{X}$ is adjusted to Krull dimension it follows that the Cousin complex $\mathcal{K}_{X}:=\mathrm{E} \mathcal{R}_{X}$ is a residual complex. Thus for every $i$ we have $\mathcal{K}_{X}^{-i}=\bigoplus \mathcal{K}_{X}(x)$, where $x$ runs over the points with $\operatorname{dim} \overline{\{x\}}=i$. Each $\mathcal{K}_{X}(x)$ is a quasi-coherent $\mathcal{O}_{X}$-module with support the closed set $\overline{\{x\}}$, and as module it is an injective hull of the residue field $\boldsymbol{k}(x)$ as $\mathcal{O}_{X, x}$-module. Since each $\mathcal{K}_{X}^{-i}$ is an injective $\mathcal{O}_{X}$-module it follows that $\mathrm{D} \mathcal{M} \cong \mathcal{H o m}_{\mathcal{O}_{X}}\left(\mathcal{M}, \mathcal{K}_{X}\right)$.

Let us prove that (ii) implies (iii). Let $\mathcal{N}$ be the coherent sheaf $\mathrm{H}^{0} \mathrm{D} \mathcal{M}$; so $\mathcal{M} \cong \mathrm{D} \mathcal{N}$ in $\mathrm{D}\left(\operatorname{Mod} \mathcal{O}_{X}\right)$. For every $i$ the sheaf

$$
\mathcal{H o m}_{\mathcal{O}_{X}}\left(\mathcal{N}, \mathcal{K}_{X}^{-i}\right) \cong \underset{\operatorname{dim} \frac{\bigoplus}{\{x\}}=i}{ } \mathcal{H o m}_{\mathcal{O}_{X}}\left(\mathcal{N}, \mathcal{K}_{X}(x)\right)
$$

is flasque and pure of dimension $i$ (or it is 0 ). Therefore

$$
\operatorname{EHom}_{\mathcal{O}_{X}}\left(\mathcal{N}, \mathcal{K}_{X}\right)=\mathcal{H o m}_{\mathcal{O}_{X}}\left(\mathcal{N}, \mathcal{K}_{X}\right) .
$$

We conclude that $\mathrm{E} \mathcal{M} \cong \mathrm{ED} \mathcal{N} \cong \mathrm{D} \mathcal{N} \cong \mathcal{M}$ in $\mathrm{D}\left(\operatorname{Mod} \mathcal{O}_{X}\right)$.

Now let us prove that (iii) implies (i). We are assuming that $\mathrm{E} \mathcal{M} \cong \mathcal{M}$ in $\mathrm{D}\left(\operatorname{Mod} \mathcal{O}_{X}\right)$. It suffices to prove that $M \in{ }^{p} \mathrm{D}_{\mathrm{f}}^{\mathrm{b}}(\operatorname{Mod} C)^{0}$, where $C:=\Gamma\left(X, \mathcal{O}_{X}\right)$ and $M:=\mathrm{R} \Gamma(X, \mathcal{M})$. By [YZ4 Lemma 7.8] it is enough to show that $\operatorname{dim} \mathrm{H}^{-i} M \leq i$ and $\mathrm{H}_{\mathrm{M}_{i}}^{j} M=0$ for all $i$ and all $j<-i$. Because $\mathrm{R} \Gamma(X,-)$ can be computed using flasque resolutions we get $M \cong \Gamma(X, \mathrm{E} \mathcal{M})$. But $\operatorname{dim} \Gamma(X, \mathrm{E} \mathcal{M})^{-i} \leq i$, and hence $\operatorname{dim} \mathrm{H}^{-i} M \leq i$. For the vanishing we use the fact that $\mathrm{R} \Gamma_{\mathrm{M}_{i}}$ can be computed by flasque resolutions (cf. [YZ3, Definition 1.13]), and that each $C$-module $\Gamma(X, \mathrm{E} \mathcal{M})^{-i}$ is a flasque $C$-module and is pure of $\operatorname{dim}=i$ (unless it is zero). This gives

$$
\mathrm{R} \Gamma_{\mathrm{M}_{i}} M \cong \Gamma_{\mathrm{M}_{i}} \Gamma(X, \mathrm{E} \mathcal{M})=\Gamma(X, \mathrm{E} \mathcal{M})^{\geq-i} .
$$

Thus $\mathrm{H}_{\mathrm{M}_{i}}^{j} M=0$ for all $j<-i$.

Remark 6.3. Theorem 6.2 implies that the category of Cohen-Macaulay complexes is an abelian subcategory of $D_{c}^{b}\left(\operatorname{Mod} \mathcal{O}_{X}\right)$. This fact that seems to have eluded Grothendieck. Sastry has communicated to us another proof of the equivalence (iii) $\Leftrightarrow$ (ii) in Theorem 6.2 using local duality at closed points of $X$.

We now go to some noncommutative examples.

Example 6.4. Let $X$ be any separated $\mathbb{k}$-scheme of finite type and $\mathcal{A}$ a coherent $\mathcal{O}_{X}$-algebra. Then $\mathcal{A}$ is a differential quasi-coherent $\mathcal{O}_{X}$-ring of finite type; as filtration $F$ we may take the trivial filtration where $F_{-1} \mathcal{A}:=0$ and $F_{0} \mathcal{A}:=\mathcal{A}$. By Theorem 5.11 the rigid dualizing complex $\mathcal{R}_{\mathcal{A}}$ exists. In this case $\mathcal{R}_{\mathcal{A}}$ can be chosen to be $\mathrm{R} \mathcal{H o m}_{\mathcal{O}_{X}}\left(\mathcal{A}, \mathcal{R}_{X}\right)$ where $\mathcal{R}_{X} \in \mathrm{D}_{\mathrm{c}}^{\mathrm{b}}\left(\operatorname{Mod} \mathcal{O}_{X}\right)$ is the central rigid dualizing complex of $X$. If $\mathcal{A}$ happens to be an Azumaya $\mathcal{O}_{X}$-algebra then $\mathcal{R}_{\mathcal{A}} \cong \mathcal{A} \otimes \mathcal{O}_{X} \mathcal{R}_{X}$ (see YZ3, Theorem 6.2]).

Example 6.5. Suppose char $\mathbb{k}=0$ and $X$ is a smooth separated scheme. Then the ring $\mathcal{D}_{X}$ of differential operators on $X$ is a differential quasi-coherent $\mathcal{O}_{X}$-ring of finite type. As filtration $F$ we may take the order filtration, in which $F_{0} \mathcal{D}_{X}:=\mathcal{O}_{X}$, 
$F_{1} \mathcal{D}_{X}:=\mathcal{O}_{X} \oplus \mathcal{T}_{X}$, and $F_{i+1} \mathcal{D}_{X}:=F_{i} \mathcal{D}_{X} \cdot F_{1} \mathcal{D}_{X}$. Here $\mathcal{T}_{X}:=\mathcal{H o m}_{\mathcal{O}_{X}}\left(\Omega_{X / \mathbb{k}}^{1}, \mathcal{O}_{X}\right)$ is the tangent sheaf. The product turns out to be

$$
\left(X^{2}, \mathcal{D}_{X}^{\mathrm{e}}\right) \cong\left(X^{2}, \mathcal{D}_{X^{2}}\left(\mathcal{O}_{X} \otimes \omega_{X}\right)\right)
$$

where $n:=\operatorname{dim} X, \boldsymbol{\omega}_{X}:=\Omega_{X / \mathbb{k}}^{n}, \mathcal{O}_{X} \otimes \boldsymbol{\omega}_{X}:=\mathcal{O}_{X^{2}} \otimes_{\mathrm{p}_{2}^{-1} \mathcal{O}_{X}} \mathrm{p}_{2}^{-1} \boldsymbol{\omega}_{X}$ and $\mathcal{D}_{X^{2}}(-)$ is the ring of twisted differential operators. By YYe4 Proposition 2.6] the rigid dualizing complex of $\mathcal{D}_{X}$ is $\mathcal{D}_{X}[2 n] \in \mathrm{D}_{\mathrm{c}}^{\mathrm{b}}\left(\operatorname{Mod} \mathcal{D}_{X}^{\mathrm{e}}\right)$.

Example 6.6. Let $X$ be any finite type separated $\mathbb{k}$-scheme, $\mathcal{M}$ a coherent $\mathcal{O}_{X^{-}}$ module and $\mathcal{D}_{X}(\mathcal{M}):=\mathcal{D}$ if $f_{\mathcal{O}_{X} / \mathbb{k}}(\mathcal{M}, \mathcal{M})$ the ring of differential operators from $\mathcal{M}$ to itself (cf. [EGA IV]). The order filtration $F$ makes $\mathcal{D}_{X}(\mathcal{M})$ into a differential quasi-coherent $\mathcal{O}_{X}$-ring, but unless char $\mathbb{k}=0, X$ is smooth and $\mathcal{M}$ is locally free, this is usually not of finite type. However if we take $\mathcal{N}:=F_{1} \mathcal{D}_{X}(\mathcal{M})$, then the subring $\mathcal{O}_{X}\langle\mathcal{N}\rangle \subset \mathcal{D}_{X}(\mathcal{M})$ is a differential quasi-coherent $\mathcal{O}_{X}$-ring of finite type.

Example 6.7. Generalizing Example 6.5 suppose $X$ is a separated finite type $\mathbb{k}$ scheme and $\mathcal{L}$ is a coherent $\mathcal{O}_{X}$-module endowed with a $\mathbb{k}$-linear Lie bracket $[-,-]$ and an $\mathcal{O}_{X}$-linear Lie homomorphism $\alpha: \mathcal{L} \rightarrow \mathcal{T}_{X}$, satisfying the conditions stated in [YZ4 Example 2.6] on affine open sets. Such $\mathcal{L}$ is called a Lie algebroid on $X$. By YZ4 Proposition 5.17 and Corollary 5.13] the universal enveloping algebras $\mathrm{U}(C ; L)$ sheafify to a differential quasi-coherent $\mathcal{O}_{X}$-ring of finite type $\mathrm{U}\left(\mathcal{O}_{X} ; \mathcal{L}\right)$.

Now suppose char $\mathbb{k}=0$ and $X$ is a smooth scheme of dimension $n$. When $\mathcal{L}=\mathcal{T}_{X}$ then $\mathrm{U}\left(\mathcal{O}_{X} ; \mathcal{L}\right)=\mathcal{D}_{X}$ as in Example 6.5 More generally when $\mathcal{L}$ is a locally free $\mathcal{O}_{X}$-module of rank $r$ the rigid dualizing complex $\mathcal{R}_{\mathrm{U}\left(\mathcal{O}_{X} ; \mathcal{L}\right)}$ of $\mathrm{U}\left(\mathcal{O}_{X} ; \mathcal{L}\right)$ was computed by Chemla $\mathrm{Ch} 2$ for $X$ affine. Since the rigidifying isomorphism used there was canonical it glues, and we obtain

$$
\mathcal{R}_{\mathrm{U}\left(\mathcal{O}_{X} ; \mathcal{L}\right)}=\mathrm{U}\left(\mathcal{O}_{X} ; \mathcal{L}\right) \otimes_{\mathcal{O}_{X}} \Omega_{X / \mathbb{k}}^{n} \otimes_{\mathcal{O}_{X}}\left(\bigwedge_{\mathcal{O}_{X}}^{r} \mathcal{L}\right)[n+r]
$$

Example 6.9. This is a special case of Example 6.7 Suppose char $\mathbb{k}=p>0$ and $X$ is smooth of dimension $n$. The sheaf of rings $\mathcal{A}:=\mathrm{U}\left(\mathcal{O}_{X} ; \mathcal{T}_{X}\right)$ is called the sheaf of crystalline differential operators (see BFG]). There is a ring homomorphism $\mathcal{A} \rightarrow \mathcal{D}_{X}$, which is neither injective nor surjective. Also $\mathcal{A}$ is finite over its center. Equation [6.8 shows that the rigid dualizing complex of $\mathcal{A}$ is $\mathcal{A}[2 n]$, just like in Example 6.5

Example 6.10. Assume char $\mathbb{k}=0$. Let $C:=\mathbb{k}[x]$ with $x$ an indeterminate, and $A:=\mathcal{D}(C)$ the first Weyl algebra. Writing $y:=\frac{\partial}{\partial x} \in A$ we have $A \cong$ $\mathbb{k}\langle x, y\rangle /([y, x]-1)$. Consider the filtration $F$ on $A$ in which $\operatorname{deg}^{F}(x)=\operatorname{deg}^{F}(y)=1$, and let $\tilde{A}:=\operatorname{Rees}^{F} A$. Then $\tilde{A}$ is the homogenized Weyl algebra, with linear generators $u:=x t, v:=y t$ and $t$, such that $t$ is central and $[v, u]=t^{2}$. Inside $\tilde{A}$ we have the commutative subring $\tilde{C}:=\operatorname{Rees}^{F} C \cong \mathbb{k}[u, t]$.

Since $\tilde{A}$ is a differential $\tilde{C}$-ring the localizations $\tilde{A}_{t}$ and $\tilde{A}_{u}$ exist. Their degree 0 components glue to a quasi-coherent sheaf of $\operatorname{rings} \mathcal{A}$ on $\operatorname{Proj} \tilde{C} \cong \mathbf{P}_{\mathbb{k}}^{1}$. We obtain a differential quasi-coherent ringed $\mathbb{k}$-scheme of finite type $\left(\mathbf{P}^{1}, \mathcal{A}\right)$. The restriction to the open set $\{t \neq 0\}$ recovers the ringed scheme $\left(\mathbf{A}^{1}, \mathcal{D}_{\mathbf{A}^{1}}\right)$, and in particular $\Gamma(\{t \neq 0\}, \mathcal{A})=A$. Thus $\left(\mathbf{P}^{1}, \mathcal{A}\right)$ can be viewed as a partial compactification of $\left(\mathbf{A}^{1}, \mathcal{D}_{\mathbf{A}^{1}}\right)$. Note that the ringed scheme $\left(\mathbf{P}^{1}, \mathcal{A}\right)$ is not isomorphic to $\left(\mathbf{P}^{1}, \mathcal{D}_{\mathbf{P}^{1}}\right)$. Indeed, consider the affine open set $W:=\{u \neq 0\} \subset \mathbf{P}^{1}$. Let $z:=u^{-1} t$, so $\Gamma\left(W, \mathcal{O}_{\mathbf{P}^{1}}\right)=\mathbb{k}[z], \Gamma(W, \mathcal{A})=\mathbb{k}\left\langle z, u^{-1} v\right\rangle$ and $\Gamma\left(W, \mathcal{D}_{\mathbf{P}^{1}}\right)=\mathbb{k}\left\langle z, \frac{\partial}{\partial z}\right\rangle$. A calculation 
shows that $u^{-1} v=-z^{3} \frac{\partial}{\partial z}$, and that the subring $\mathbb{k}\left\langle z,-z^{3} \frac{\partial}{\partial z}\right\rangle$ is not isomorphic as $\mathbb{k}[z]$-ring to the Weyl algebra $\mathbb{k}\left\langle z, \frac{\partial}{\partial z}\right\rangle$.

Example 6.11. Consider another geometric object associated to the situation of Example 6.10 the projective spectrum Proj $\tilde{A}$ in the sense of Artin-Zhang AZ. We claim that $\left(\mathbf{P}^{1}, \mathcal{A}\right)$ is an "open subscheme" of the "complete surface" $\operatorname{Proj} \tilde{A}$, whose complement consists of one point. To make this statement precise we have to pass to abelian categories. Recall that $\mathrm{QGr} \tilde{A}$ is the quotient category $\operatorname{GrMod} \tilde{A} /\{\mathfrak{m}$-torsion\}, where $\mathfrak{m}$ is the augmentation ideal of $\tilde{A}$; and $\operatorname{Proj} \tilde{A}$ is the geometric object such that "QCoh Proj $\tilde{A}=\mathrm{QGr} \tilde{A}$ ". Because QCoh $\mathcal{A}$ is obtained by gluing the categories QCoh $\left.\mathcal{A}\right|_{\{t \neq 0\}}$ and $\left.\mathrm{QCoh} \mathcal{A}\right|_{\{u \neq 0\}}$, we see that QCoh $\mathcal{A}$ is equivalent to the quotient of $\mathrm{QGr} \tilde{A}$ by the localizing subcategory $\{\mathfrak{a}$-torsion\}, where $\mathfrak{a}$ is the two-sided ideal $(t, u) \subset \tilde{A}$.

Let's continue with the setup of the last two examples. The subcategory of noetherian objects in $\mathrm{QGr} \tilde{A}$ is denoted by qgr $\tilde{A}$; it is equivalent to the category $\operatorname{GrMod}_{\mathrm{f}} \tilde{A} /\{\mathfrak{m}$-torsion $\}$. In [KKO there is a duality

$$
\mathrm{D}_{\mathrm{qgr}}: \mathrm{D}^{\mathrm{b}}(\mathrm{qgr} \tilde{A}) \rightarrow \mathrm{D}^{\mathrm{b}}\left(\mathrm{qgr} \tilde{A}^{\mathrm{op}}\right) \text {. }
$$

The formula (with a slight adjustment) is given below. Let us denote by $\pi$ : $\operatorname{GrMod} \tilde{A} \rightarrow \mathrm{QGr} \tilde{A}$ the localization functor and by $\tilde{\Gamma}: \mathrm{QGr} \tilde{A} \rightarrow \operatorname{GrMod} \tilde{A}$ its adjoint

$$
\tilde{\Gamma} \mathcal{M}:=\bigoplus_{i \geq 0} \operatorname{Hom}_{\mathrm{QGr} \tilde{A}}(\pi \tilde{A}(-i), \mathcal{M}) .
$$

Denote by $\pi^{\mathrm{op}}$ and $\tilde{\Gamma}^{\mathrm{op}}$ the corresponding right versions. Let $R_{\tilde{A}}$ be the balanced dualizing complex of $\tilde{A}$; so $R_{\tilde{A}} \cong \boldsymbol{\omega}_{\tilde{A}}[3]$ where $\boldsymbol{\omega}_{\tilde{A}}$ is the dualizing bimodule of $\tilde{A}$. Then

$$
\mathrm{D}_{\mathrm{qgr}} \mathcal{M}:=\pi^{\mathrm{op}} \mathrm{RHom}_{\tilde{A}}^{\mathrm{gr}}\left(\mathrm{R} \tilde{\Gamma} \mathcal{M}, R_{\tilde{A}}^{[-1]}\right) .
$$

With this normalization the duality $\mathrm{D}_{\mathrm{qgr}}$ is compatible with the global $\mathbb{k}$-linear duality, namely

$$
\operatorname{RHom}_{\mathrm{QGr} \tilde{A}}(\pi \tilde{A}, \mathcal{M})^{*} \cong \mathrm{RHom}_{\mathrm{QGr} \tilde{A} \text { op }}\left(\pi^{\mathrm{op}} \tilde{A}^{\mathrm{op}}, \mathrm{D}_{\mathrm{qgr}} \mathcal{M}\right) .
$$

(Actually the formula (6.12) gives a duality for any noetherian connected graded $\mathbb{k}$-algebra $\tilde{A}$ admitting a balanced dualizing complex, be it regular or not; cf. [YZ1.)

Kazhdan has recently asked us whether the duality $\mathrm{D}_{\mathrm{qgr}}$ is compatible with the duality on $\left(\mathbf{P}^{1}, \mathcal{A}\right)$ via the "open embedding" $g:\left(\mathbf{P}^{1}, \mathcal{A}\right) \hookrightarrow \operatorname{Proj} \tilde{A}$. We can answer positively:

Proposition 6.13. The diagram

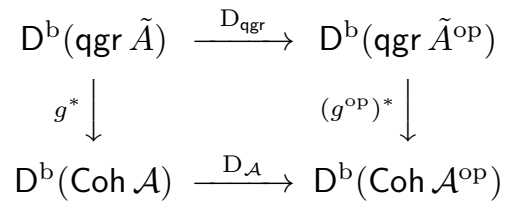

where $\mathrm{D}_{\mathcal{A}}$ is the duality determined by the rigid dualizing complex $\mathcal{R}_{\mathcal{A}}$, is commutative.

Proof. Consider the graded bimodule $\tilde{A}(i)$ for any integer $i$. It is generated by the element 1 which is in degree $-i$. When we invert $t$ we get a generator $t^{i} \in\left(\tilde{A}(i)_{t}\right)_{0}$. 
Likewise when we invert $u$ we get a generator $u^{i} \in\left(\tilde{A}(i)_{u}\right)_{0}$. Therefore we obtain a sheaf $\mathcal{A}(i)$ of $\mathcal{A}$-bimodules on $\mathbf{P}^{1}$, such that

$$
\left.\mathcal{A}(i)\right|_{\{t \neq 0\}}=\left.\left.\mathcal{A}\right|_{\{t \neq 0\}} \cdot t^{i} \cong \mathcal{A}\right|_{\{t \neq 0\}}
$$

and

$$
\left.\mathcal{A}(i)\right|_{\{u \neq 0\}}=\left.\mathcal{A}\right|_{\{u \neq 0\}} \cdot u^{i}=\left.u^{i} \cdot \mathcal{A}\right|_{\{u \neq 0\}},
$$

and the gluing on the open set $\{u t \neq 0\}$ is multiplication by $t^{-i} u^{i}=x^{i}$. Note that the $\mathcal{A}$-bimodule $\mathcal{A}(i)$ is not locally centrally generated if $i \neq 0$.

Since $\mathcal{A}(i)$ is a differential $\mathcal{O}_{\mathbf{P}^{1}}$-bimodule we can view it as a coherent $\mathcal{A}^{\mathrm{e}}$-module supported on the diagonal $\Delta\left(\mathbf{P}^{1}\right) \subset\left(\mathbf{P}^{1}\right)^{2}$. As left $\mathcal{A}$-module we have $\mathcal{A}(i) \cong$ $g^{*} \pi \tilde{A}(i)$; and likewise on the right.

Let us compute the balanced dualizing complex $R_{\tilde{A}}$. As mentioned above, since $\tilde{A}$ is an AS-regular algebra it follows that $R_{\tilde{A}} \cong \boldsymbol{\omega}_{\tilde{A}}[3]$, and $\boldsymbol{\omega}_{\tilde{A}}=\tilde{A}^{\sigma}(-3)$ for some graded algebra automorphism $\sigma$. See [Ye4, Proposition 1.1]. By [Ye4, Corollary 3.6] the automorphism $\sigma$ is trivial on the center, so it is $\mathbb{k}[t]$-linear. Now $\left(\tilde{A}_{t}\right)_{0} \cong A$, and we know that $R_{A} \cong A[2]$ by [Ye4 Theorem 2.6]. Hence $\sigma$ is in fact trivial and $R_{\tilde{A}} \cong \tilde{A}(-3)[3]$.

Let us denote $B:=\Gamma(\{u \neq 0\}, \mathcal{A})=\left(\tilde{A}_{u}\right)_{0}$. The localization of rigid dualizing complexes (see [YZ4, Theorem 6.2]) shows that the rigid dualizing complex of $B$ is

$$
R_{B} \cong\left(\left(R_{\tilde{A}}\right)_{u}\right)_{0}[-1] \cong\left(\tilde{A}(-3)[2]_{u}\right)_{0} \cong B u^{-3}[2]
$$

and hence

$$
\left.\left.\mathcal{R}_{\mathcal{A}}\right|_{\{u \neq 0\}} \cong \mathcal{A}(-3)[2]\right|_{\{u \neq 0\}} .
$$

On the open set $\{t \neq 0\}$ we have

$$
\left.\left.\left.\mathcal{R}_{\mathcal{A}}\right|_{\{t \neq 0\}} \cong \mathcal{A}[2]\right|_{\{t \neq 0\}} \cong \mathcal{A}(-3)[2]\right|_{\{t \neq 0\}} .
$$

The bimodule $\left.\left.\mathcal{A}\right|_{\{t u \neq 0\}} \cong \mathcal{A}(-3)\right|_{\{t u \neq 0\}}$ has only constant automorphisms (because $\left.\mathrm{Z}\left(A_{x}\right)=\mathbb{k}\right)$, so it follows that $\mathcal{R}_{\mathcal{A}} \cong \mathcal{A}(-3)[2]$ in $\mathrm{D}\left(\operatorname{Mod} \mathcal{A}^{\mathrm{e}}\right)$.

The category $\mathrm{D}^{\mathrm{b}}(\mathrm{qgr} \tilde{A})$ is a quotient of $\mathrm{D}^{\mathrm{b}}\left(\operatorname{GrMod}_{\mathrm{f}} \tilde{A}\right)$. We know that $\tilde{A}$ is regular, and hence any object in $\mathrm{D}^{\mathrm{b}}\left(\operatorname{GrMod}_{\mathrm{f}} \tilde{A}\right)$ is isomorphic to a bounded complex of finite free graded modules. Now for any $i$ we get

$$
\left(g^{\mathrm{op}}\right)^{*} \mathrm{D}_{\mathrm{qgr}} \pi \tilde{A}(i) \cong \mathrm{D}_{\mathcal{A}} g^{*} \pi \tilde{A}(i) \cong \mathcal{A}(-3-i)[2]
$$

in $\mathrm{D}^{\mathrm{b}}\left(\operatorname{Coh} \mathcal{A}^{\text {op }}\right)$. Maps $\tilde{A}(i) \rightarrow \tilde{A}(j)$ in $\operatorname{GrMod}_{\mathrm{f}} \tilde{A}$ are right multiplication by elements $a \in \tilde{A}(j-i)$, and these are sent under both dualities to left multiplication by the same $a$. Hence $\left(g^{\text {op }}\right)^{*} \mathrm{D}_{\mathrm{qgr}} \mathcal{P} \cong \mathrm{D}_{\mathcal{A}} g^{*} \mathcal{P}$ for any object $\mathcal{P} \in \mathrm{D}^{\mathrm{b}}(\mathrm{qgr} \tilde{A})$. Likewise for morphisms.

If $A$ is a finite $\mathbb{k}$-algebra of finite global dimension then the rigid dualizing complex of $A$ is $A^{*}:=\operatorname{Hom}_{\mathbb{k}}(A, \mathbb{k})$. It is known that $M \mapsto A^{*} \otimes_{A}^{\mathrm{L}} M$ is the Serre functor of $\mathrm{D}_{\mathrm{f}}^{\mathrm{b}}(\operatorname{Mod} A)$. Namely $A^{*} \otimes_{A}^{\mathrm{L}}$ - is an auto-equivalence of $\mathrm{D}_{\mathrm{f}}^{\mathrm{b}}(\operatorname{Mod} A)$, and there is a bifunctorial nondegenerate pairing

$$
\operatorname{Hom}_{\mathrm{D}(\operatorname{Mod} A)}(M, N) \times \operatorname{Hom}_{\mathrm{D}(\operatorname{Mod} A)}\left(N, A^{*} \otimes_{A}^{\mathrm{L}} M\right) \rightarrow \mathbb{k}
$$

for $M, N \in \mathrm{D}_{\mathrm{f}}^{\mathrm{b}}(\operatorname{Mod} A)$. Cf. [MY] and $[\mathrm{BO}$. Likewise, for a smooth $n$-dimensional projective scheme $X$ the rigid dualizing complex is $\Omega_{X / \mathbb{k}}^{n}[n]$ (see Example 3.8), and the Serre functor is $\mathcal{M} \mapsto \Omega_{X / \mathbb{k}}^{n}[n] \otimes_{\mathcal{O}_{X}} \mathcal{M}$. Kontsevich has asked us whether this is true in greater generality. We have the following partial answer. 
Let $\mathcal{A}$ be a coherent $\mathcal{O}_{X}$-ring. We say that $\mathcal{A}$ is regular if for any affine open set $U$ the ring $\Gamma(U, \mathcal{A})$ has finite global dimension. As explained in Example 6.4 the rigid dualizing complex $\mathcal{R}_{\mathcal{A}}$ can be assumed to live in $\mathrm{D}_{\mathrm{c}}^{\mathrm{b}}\left(\operatorname{Mod} \mathcal{A} \otimes_{\mathcal{O}_{X}} \mathcal{A}^{\mathrm{op}}\right)$.

Proposition 6.14. Let $X$ be a projective $\mathbb{k}$-scheme, and let $\mathcal{A}$ be a regular coherent $\mathcal{O}_{X}$-algebra, with rigid dualizing complex $\mathcal{R}_{\mathcal{A}} \in \mathrm{D}_{\mathrm{c}}^{\mathrm{b}}\left(\operatorname{Mod} \mathcal{A} \otimes_{\mathcal{O}_{X}} \mathcal{A}^{\mathrm{op}}\right)$. Then $\mathcal{M} \mapsto$ $\mathcal{R}_{\mathcal{A}} \otimes_{\mathcal{A}}^{\mathrm{L}} \mathcal{M}$ is a Serre functor of $\mathrm{D}_{\mathrm{c}}^{\mathrm{b}}(\operatorname{Mod} \mathcal{A})$.

Proof. Choose an ample $\mathcal{O}_{X}$-module $\mathcal{L}$, and let $\mathcal{M}(1):=\mathcal{M} \otimes_{\mathcal{O}_{X}} \mathcal{L}$. Define $\tilde{A}:=$ $\mathbb{k} \oplus \bigoplus_{i>1} \Gamma(X, \mathcal{A}(i))$, which is a connected graded noetherian $\mathbb{k}$-algebra, finite over its center. Then $\operatorname{Coh} \mathcal{A} \cong \operatorname{qgr} \tilde{A}$, and this category has finite global dimension. Let $R_{\tilde{A}}$ be the balanced dualizing complex of $\tilde{A}$. Then $\mathcal{R}_{\mathcal{A}} \cong \pi R_{\tilde{A}}[-1]$, and the claim follows from [NV Theorem A.4].

\section{REFERENCES}

[AKO] D. Auroux, L. Katzarkov and D. Orlov, Mirror symmetry for weighted projective planes and their noncommutative deformations, eprint math.AG/0404281 at http://arxiv.org

[AJL] L. Alonso, A. Jeremías and J. Lipman, Duality and flat base change on formal schemes, in "Studies in Duality on Noetherian Formal Schemes and Non-Noetherian Ordinary Schemes," Contemp. Math. 244, Amer. Math. Soc., 1999, 3-90.

[AZ] M. Artin and J.J. Zhang, Noncommutative projective schemes, Adv. Math. 109 (1994), $228-287$.

[Be] A.A. Beilinson, The derived category of coherent sheaves on $\mathbf{P}^{n}$, Selecta Math. Soviet. 3 (1983/84), no. 3, 233-237.

[BO] A. Bondal and D. Orlov, Derived categories of coherent sheaves, Proceedings of the International Congress of Mathematicians, Vol. II (Beijing, 2002), 47-56, Higher Ed. Press, Beijing, 2002.

[Bor] A. Borel et al., Algebraic D-modules, Perspectives in Mathematics, 2. Academic Press, Inc., Boston, MA, 1987.

[BBD] A.A. Beilinson, J. Bernstein and P. Deligne, Faisceaux pervers, in "Analysis and topology on singular spaces, I" (Luminy, 1981), Astérisque, 100, Soc. Math. France, Paris, (1982), 5-171.

[BFG] R. Bezrukavnikov, M. Finkelberg and V. Ginzburg, Cherednik algebras and Hilbert schemes in characteristic $p$, eprint math.RT/0312474 at http://arXiv.org

[Br] T. Bridgeland, Flops and derived categories, Invent. Math. 147 (2002), no. 3, 613-632.

[Bz] R. Bezrukavnikov, Perverse coherent sheaves (after Deligne), eprint math.AG/0005152 at http://arXiv.org

[Ch1] S. Chemla, A duality property for complex Lie algebroids, Math. Z. 232 (1999), 367388.

[Ch2] S. Chemla, Rigid dualizing complexes for quantum enveloping algebras and algebras of generalized differential operators, J. Algebra 276 (2004), no. 1, 80-102.

[Co] B. Conrad, "Grothendieck Duality and Base Change," Lecture Notes in Math. 1750, Springer, 2000.

[Do] M.R. Douglas, Dirichlet branes, homological mirror symmetry, and stability, Proceedings of the International Congress of Mathematicians, Vol. III (Beijing, 2002), 395-408, Higher Ed. Press, Beijing, 2002.

[EG] P. Etingof and V. Ginzburg, Symplectic reflection algebras, Calogero-Moser space, and deformed Harish-Chandra homomorphism, Invent. Math. 147 (2002), no. 2, 243-348.

[EGA I] A. Grothendieck and J. Dieudonné, "Éléments de Géometrie Algébrique I," Springer, Berlin, 1971.

[EGA IV] A. Grothendieck and J. Dieudonné, "Éléments de Géometrie Algébrique IV," Publ. Math. IHES 32 (1967).

[GJ] K.R. Goodearl and D.A. Jordan, Localizations of injective modules, Proc. Edinburgh Math. Soc. (2) 28 (1985), no. 3, 289-299.

[Ha] R. Hartshorne, "Algebraic geometry," Springer-Verlag, New York-Heidelberg, 1977. 
[HK] R. Hübl and E. Kunz, Regular differential forms and duality for projective morphisms, J. reine angew. Math. 410 (1990), 84-108.

[Jo] P. Jørgensen Non-commutative graded homological identities, J. London Math. Soc. 57 (1998), 336-350.

[Ka] M. Kashiwara, A t-structure of the derived category of holonomic D-modules, eprint math.AG/0302086 at http://arXiv.org

[KKO] A. Kapustin, A. Kuznetsov and D. Orlov, Noncommutative Instantons and Twistor Transform, Comm. Math. Phys. 221 (2001), 385-432.

[Kl] S.L. Kleiman, Relative duality for quasi-coherent sheaves, Compositio Math. 41 (1980), no. $1,39-60$.

[KS] M. Kashiwara and P. Schapira, "Sheaves on Manifolds," Springer-Verlag, Berlin, 1990.

[Li] J. Lipman, Dualizing sheaves, differentials and residues on algebraic varieties, Astérisque 117 (1984).

[LMB] G. Laumon and L. Moret-Bailly, "Champs algebriques," Springer, 2000.

[LR] T.H. Lenagan and L. Rigal, Quantum graded algebras with a straightening law and the AS-Cohen-Macaulay property for quantum determinantal rings and quantum grassmannians, eprint math.QA/0403021 at http://arxiv.org

[Lu] V. Lunts, Deformations of quasicoherent sheaves of algebras, J. Algebra 259 (2003), no. $1,59-86$

[MR] J.C. McConnell and J.C. Robson, "Noncommutative Noetherian Rings," Wiley, Chichester, 1987.

[Mu S. Mukai, Duality between $D(X)$ and $D(\hat{X})$ with its application to Picard sheaves, Nagoya Math. J. 81 (1981), 153-175.

[MY] J. Miyachi and A. Yekutieli, Derived Picard groups of finite dimensional hereditary algebras, Compositio Math. 129 (2001), 341-368.

[Ne] A. Neeman, The Grothendieck duality theorem via Bousfield's techniques and Brown representability, J. Amer. Math. Soc. 9 (1996), no. 1, 205-236.

[NV] K. de Naeghel and M. Van den Bergh, Ideal classes of three-dimensional Sklyanin algebras, J. Algebra 276 (2004), 515-551.

[RD] R. Hartshorne, "Residues and Duality," Lecture Notes in Math. 20, Springer-Verlag, Berlin, 1966.

[Ri] G.S. Rinehart, Differential forms on general commutative algebras, Trans. Amer. Math. Soc. 108 (1963), 195-222.

[Si] L. Silver, Noncommutative localizations and applications, J. Algebra 7 (1967), 44-76.

[VdB1] M. Van den Bergh, Existence theorems for dualizing complexes over non-commutative graded and filtered ring, J. Algebra 195 (1997), no. 2, 662-679.

[VdB2] M. Van den Bergh, Three-dimensional flops and non-commutative rings, Duke Math. J. 122 (2004), no. 3, 423-455.

[WZ] Q.S. Wu and J.J. Zhang, Some homological invariants of local PI algebras, J. Algebra 225 (2000), no. 2, 904-935.

[Ye1] A. Yekutieli, Dualizing complexes over noncommutative graded algebras, J. Algebra 153 (1992), 41-84.

[Ye2] A. Yekutieli, Residues and differential operators on schemes, Duke Math. J. 95 (1998), 305-341.

[Ye3] A. Yekutieli, Dualizing complexes, Morita equivalence and the derived Picard group of a ring, J. London Math. Soc. (2) 60 (1999), no. 3, 723-746.

[Ye4] A. Yekutieli, The rigid dualizing complex of a universal enveloping algebra, J. Pure Appl. Algebra 150 (2000), 85-93.

[YZ1] A. Yekutieli and J.J. Zhang, Serre duality for noncommutative projective schemes, Proc. Amer. Math. Soc. 125 (1997), 697-707.

[YZ2] A. Yekutieli and J.J. Zhang, Rings with Auslander dualizing complexes, J. Algebra 213 (1999), no. 1, 1-51.

[YZ3] A. Yekutieli and J.J. Zhang, Residue Complexes over Noncommutative Rings, J. Algebra 259 (2003) no. 2, 451-493.

[YZ4] A. Yekutieli and J.J. Zhang, Dualizing Complexes and Perverse Modules over Differential Algebras, Compositio Math. 141 (2005), 620-654.

[YZ5] A. Yekutieli and J.J. Zhang, Rigid Dualizing Complexes on Schemes, eprint math.AG/0405570 at http://arXiv.org 
A. Yekutieli: Department of Mathematics Ben Gurion University, Be’er Sheva 84105, ISRAEL

E-mail address: amyekut@math.bgu.ac.il

J.J. Zhang: Department of Mathematics, Box 354350, University of Washington, Seattle, Washington 98195, USA

E-mail address: zhang@math.washington.edu 